NIST GCR 09-922

\title{
Radiation Transport Measurements in Methanol Pool Fires with Fourier Transform Infrared Spectroscopy
}

\author{
Aykut Yilmaz \\ Department of Mechanical Engineering \\ University of Maryland \\ College Park, MD 20742
}



NIST GCR 09-922

\section{Radiation Transport Measurements in Methanol Pool Fires with Fourier Transform Infrared Spectroscopy}

Prepared for

U.S. Department of Commerce Building and Fire Research Laboratory National Institute of Standards and Technology

Gaithersburg, MD 20899-8660

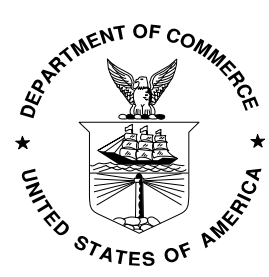

U.S. Department of Commerce Carlos M. Gutierrez, Secretary 


\section{Notice}

This report was prepared for the Building and Fire Research Laboratory of the National Institute of Standards and Technology under Grant number 70NANB4H1130. The statement and conclusions contained in this report are those of the authors and do not necessarily reflect the views of the National Institute of Standards and Technology or the Building and Fire Research Laboratory. 


\section{ABSTRACT}

Title of Document:

Directed By:

\section{RADIATION TRANSPORT MEASUREMENTS IN METHANOL POOL FIRES WITH FOURIER TRANSFORM INFRARED SPECTROSCOPY}

Aykut Yilmaz, Master of Science, 2008

Associate Professor Gregory Jackson

Department of Mechanical Engineering

Pool fires rely on heat feedback from the combustion process to the liquid surface to vaporize the fuel. This coupled relationship determines the fuel burning rate and thus the fire structure and size. Radiative heat transfer is the dominant heat feedback in large pool fires. Species concentrations and temperatures have large influence on the radiative heat transfer in the fuel rich-core between the flame and the pool surface. To study radiative transport in the fuel-rich core, an experimental method was developed to measure spectral absorption through various pathlengths inside a $30 \mathrm{~cm}$ diameter methanol pool fire by using a Fourier Transform Infrared Spectrometer with $\mathrm{N}_{2}$ purged optical probes. The measured spectra are used to estimate species concentration profiles 
of methanol, $\mathrm{CO}$, and $\mathrm{CO}_{2}$ in the fuel rich core by fitting predictions of a spectrally resolved radiation transport model to the measured spectra. Results show the importance of reliable temperature measurements for fitting the data and the need for further measurements to further understand the structure of fuel rich cores in pool fires. 


\title{
RADIATION TRANSPORT MEASUREMENTS IN METHANOL POOL FIRES WITH FOURIER TRANSFORM INFRARED SPECTROSCOPY
}

\author{
By \\ Aykut Yilmaz \\ Thesis submitted to the Faculty of the Graduate School of the \\ University of Maryland, College Park, in partial fulfillment \\ of the requirements for the degree of \\ Master of Science \\ 2008
}

Advisory Committee:

Associate Professor Gregory Jackson, Chair

Associate Professor André Marshall

Associate Professor Arnaud Trouvé

Dr. Anthony Hamins

Dr. Marc Nyden 
(C) Copyright by Aykut Yilmaz 2008 


\section{Acknowledgements}

First and foremost, I would like to express my sincere appreciation and thanks to those who supported me personally during my studies. My parents gave me their continuous support, professional and personal, during my graduate studies and everything preceding it. My father, Emin, provided me equipment, office space, advice, technical experience, and financial support when needed, all of which have made my graduation possible. Equally as important, my mother, Emel, provided emotional support and countless home-cooked meals to help keep my focus on my studies. My brother, Bülent, has helped me deal with challenges over the past several years too numerous to mention. Together with his wife, Leslie, they have been my local support as I have attended the University of Maryland.

I also wish to express my thanks to those who have helped support me academically. The funding for my research was provided by the National Institute of Standards and Technology (NIST), Project \#70NANB4H1130, managed by Dr. Jiann Yang. My advisor, Dr. Gregory Jackson, provided his patience, advice, and supporting effort as I worked through my thesis, and I am grateful for his professional guidance for my pursuits both inside and outside of university life. I would also like to express my thanks to Dr. Anthony Hamins and Dr. Marc Nyden of NIST for educating me about pool fires and IR spectroscopy, helping me develop the experiment, providing me the opportunity to carry out my research, and serving on my thesis committee. I acknowledge and appreciate the help of Dr. Andrew Lock of NIST, who set up and ran the gas chromatograph used in the experiment, and also provided his expertise in combustion experiments. I am also appreciative to Dr. André Marshall and Dr. Arnaud 
Trouvé of the Department of Fire Protection Engineering at the University of Maryland for serving on my thesis committee.

There is an extensive supporting group from the University of Maryland and NIST to which I owe thanks. From NIST, Mr. Marco Fernandez deserves special recognition for lending his knowledge of construction and manufacturing, and for providing his time to help with the design and machining of several components of my test rig. I am appreciative of the friendship I developed with him over my tenure as a guest researcher. I am also grateful to Dr. Kaoru Wakatsuki. With his gracious help at the beginning, I was able to build upon his research on the effect of infrared absorption in pool fires. I would like to thank the many people who consistently helped me conduct my experiments or provided help with tools or materials, Dr. Gwon Hyun Ko, Dr. Sung Chan Kim, Mr. Joshua Dinaburg, Mr. Alex Maranghides, Mr. Richard Harris, and Mr. Laurean DeLauter of the NIST Fire Research Division, and Mr. Howie Grossenbacher of the Department of Aerospace Engineering Machine Shop at the University of Maryland. I would also like to thank Ms. Barbara Huff and Ms. Mickey Walz of NIST and Mr. Fitz Walker and Mr. Amarildo Damata of the Mechanical Engineering Graduate Office for keeping my paperwork in order and up to date. Finally, I would like to thank Mr. Tom Beigel and Dr. David Biggio of the University of Maryland for allowing me to be their teaching assistant for their respective classes and for providing support as I needed it during my thesis work. 


\section{Table of Contents}

List of Tables .................................................................................................. vi

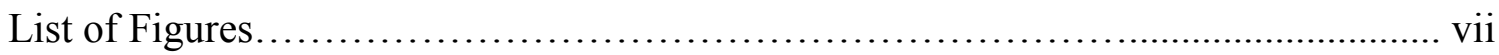

CHAPTER 1: $\quad$ Introduction............................................................................ 1

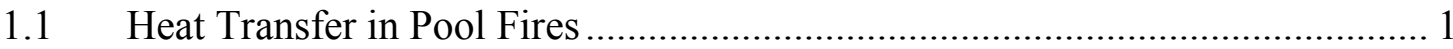

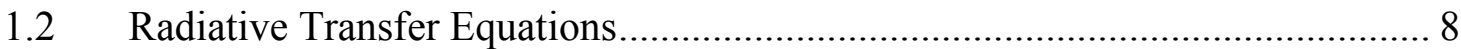

1.2.1 Fundamentals of Spectral Radiation .................................................... 10

1.2.2 Definition of absorption coefficients .................................................. 16

Use of spectroscopy in combustion studies ............................................. 18

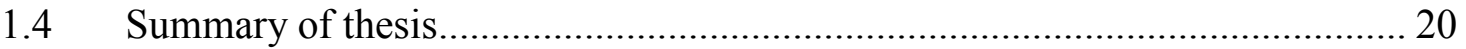

CHAPTER 2: $\quad$ Pool Fire Experimental Systems.................................................... 23

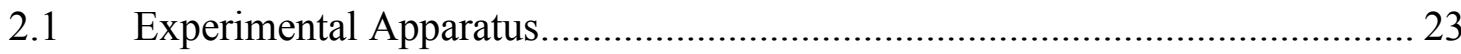

2.1.1 Optical Arrangement and Diagnostics ............................................... 27

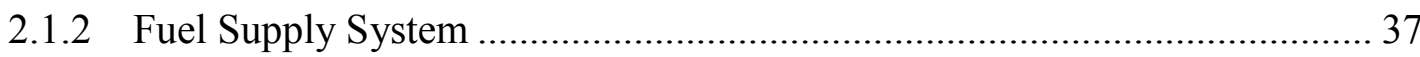

2.1.3 Gas-phase Temperature Measurements ................................................. 40

2.1.4 Gas-phase Species Measurements ....................................................... 42

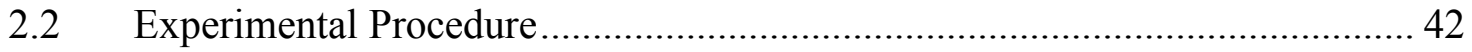

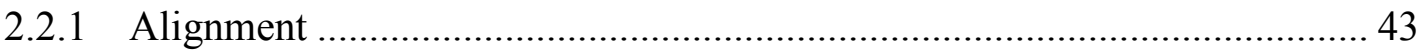

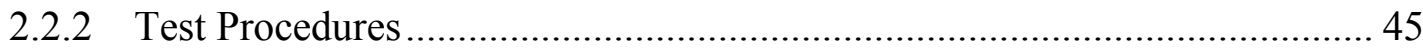

CHAPTER 3: $\quad$ Results of Methanol Pool Fire Measurements ................................. 49

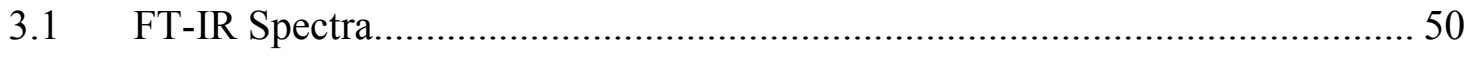

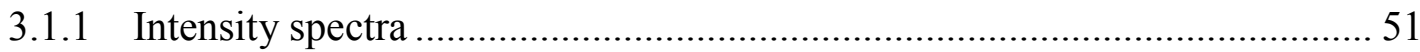




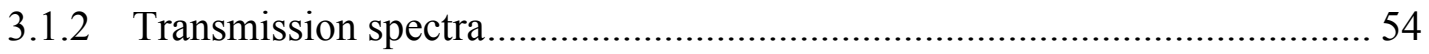

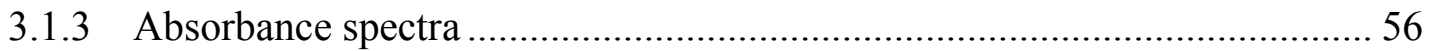

3.1.4 Correction of time-dependent instrument responses...............................5 57

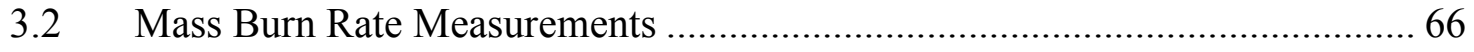

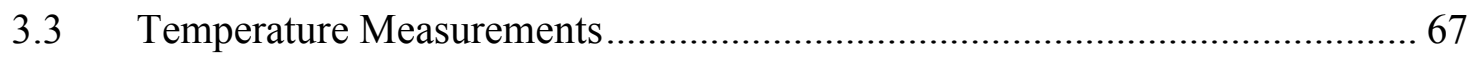

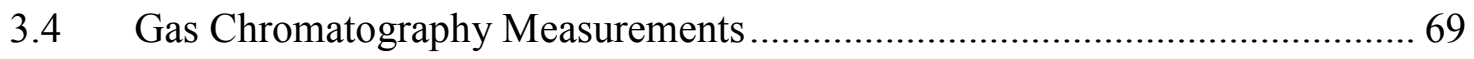

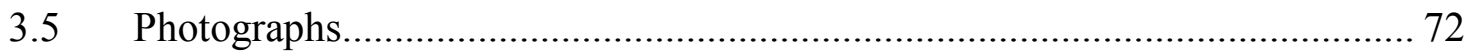

CHAPTER 4: Calculation of Species Concentrations ......................................... 75

4.1 Description of the concentration profile solver ...................................... 75

4.2 Results of the concentration profile solver .......................................... 80

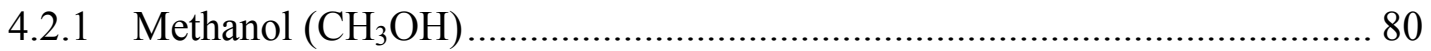

4.2.2 Carbon Dioxide $\left(\mathrm{CO}_{2}\right)$ and Carbon Monoxide $(\mathrm{CO})$.............................. 101

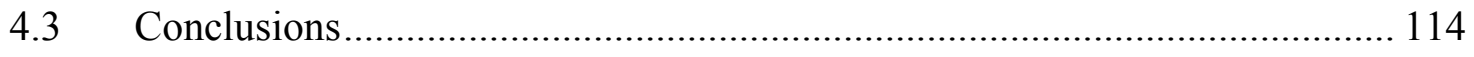

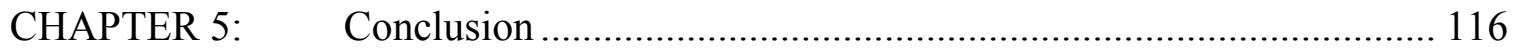

$5.1 \quad$ Recommendations for Future Research ............................................. 117

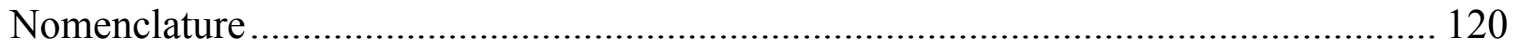

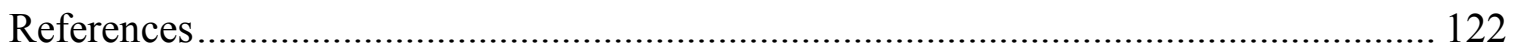




\section{List of Tables}

Table 4.1: Table of infrared bands used for species analysis of methanol pool fire....... 79

Table 4.2: Wavenumber regions used for $\mathrm{CO}$ and $\mathrm{CO}_{2}$ when solved independently ... 103 


\section{List of Figures}

Figure 1.1: Ratio of mass flux $\dot{m}^{\prime \prime}$ to the diffusive transfer number $B$ as a function of pool diameter, reproduced from Figure 12 of Hamins et al. ${ }^{5}$ (not subject to copyright)

Figure 1.2: Vibrating-rotator model of a molecule illustrating vibrational $(v)$ and rotational $(j)$ energy levels and transitions (adapted from figures by Tien, ${ }^{11}$ Brewster, ${ }^{23}$ and Wakatsuki ${ }^{17}$ ).

Figure 1.3: Absorbance peak of $\mathrm{C}_{2} \mathrm{H}_{4}$ exhibiting energy transitions of a vibrating-rotator

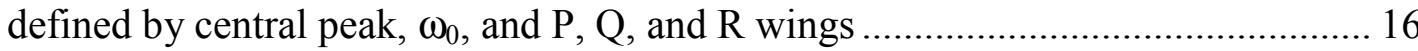

Figure 2.1: A schematic of the pool fire test rig showing the FT-IR beam path from source to detector

Figure 2.2: A schematic of the fuel pan with its attached features, including purge lines, thermocouple, and fuel input..... 27

Figure 2.3: A labeled photograph of the partially-assembled angled probe and probe extension used in pool fire experiments.

Figure 2.4: Picture of angled mirror below the fuel pan, aluminum plate and set screws used for optical alignment, and tubes used for purge containment.

Figure 2.5: Bottom view of stainless steel cone with attached $\mathrm{ZnSe}$ window and brass Swagelok $1 / 16$ " NPT to $1 / 8$ " tube fitting.

Figure 2.6: A schematic of fuel lines and valves controlling the flow of fuel during fires

Figure 3.1: Reference (background) and sample spectra from a methanol pool fire showing characteristic spectral absorption regions of a) methanol, b) $\mathrm{CO}$ and c) $\mathrm{CO}_{2}$

Figure 3.2: A plot of transmitted blackbody intensity spectra measured through various centerline heights $(z)$ of a $d=30 \mathrm{~cm}$ methanol pool fire

Figure 3.3: Plots of transmission spectra at various heights inside a $30 \mathrm{~cm}$ methanol pool fire

Figure 3.4: Plots of absorbance spectra for a $d=30 \mathrm{~cm}$ methanol pool fire at various heights

Figure 3.5: Absorbance plots illustrating correction method with a) user inputted points ('ginput') and the resulting MATLAB polynomial fit ('polyfit'), and b) the corrected spectrum 
Figure 3.6: Corrected absorbance plots for at measured heights $(z)$ for the methanol vibration-rotation absorption bands due to a) $\mathrm{C}-\mathrm{O}$ stretching, and b) $\mathrm{C}-\mathrm{H}$ stretching (with methane)

Figure 3.7: Corrected absorbance spectra for $\mathrm{CO}$ stretching and $\mathrm{CO}_{2}$ asymmetric stretching vibration-rotation absorption bands

Figure 3.8: Corrected absorbance spectra for the $\mathrm{H}_{2} \mathrm{O}$ bending vibration-rotation absorption band

Figure 3.9: Plot of fuel burn rate $(\dot{m})$ measurements versus probe height $(z)$ in $d=30$ $\mathrm{cm}$ methanol pool fire

Figure 3.10: Comparison of centerline mean temperature measurements $(T)$ versus height above fuel surface $(z)$

Figure 3.11: Molar fractions $X$ of major gas species along the centerline of a $d=30 \mathrm{~cm}$ methanol pool fire 70

Figure 3.12: Molar fractions $X$ of minor gas species along the centerline of a $d=30 \mathrm{~cm}$ methanol pool fire 71

Figure 3.13: Normalized molar fractions, $X_{\text {norm }}$, of major gas species along the centerline of a $d=30 \mathrm{~cm}$ methanol pool fire, according to eq. (3.6)

Figure 3.14: Normalized molar fractions, $X_{\text {norm }}$, of minor gas species along the centerline of a $d=30 \mathrm{~cm}$ methanol pool fire, according to eq. (3.6)...

Figure 3.15: Photographs (2) showing the methanol pool fire test rig at two different probe locations, $z$ 73

Figure 3.16: Photograph of $d=30 \mathrm{~cm}$ methanol pool fire test at probe height $z=15 \mathrm{~cm}$, illustrating probe effects 74

Figure 4.1: Temperature-dependent absorption coefficients for $\mathrm{CH}_{3} \mathrm{OH}$ used in concentration fit study.

Figure 4.2: Linearly interpolated experimental temperature profiles and pool surface conditioned cubic temperature fit used by spectra calculator for comparison to experimental spectra

Figure 4.3: Calculated spectra fits in methanol $\mathrm{C}-\mathrm{O}$ stretching band using $\mathrm{CH}_{3} \mathrm{OH}$ concentration profile from GC measurements with temperatures from the Present study, Weckman and Strong, Hamins and Gorchkov and FDS direct numerical simulation result solved with $1 \mathrm{~cm}$ grid, linearly extrapolated

Figure 4.4: Predicted $\mathrm{CH}_{3} \mathrm{OH}$ mole fraction profile when fitted to experimental spectra with surface-conditioned cubic temperature profile 
Figure 4.5: $\mathrm{CH}_{3} \mathrm{OH}$ spectra fits to experiment using temperature profile from the present study with $T(0)=T_{\text {boiling }}$ conditioned fit .......................................................... 88

Figure 4.6: Predicted $\mathrm{CH}_{3} \mathrm{OH}$ mole fraction profile (forced decay to $<80 \%$ ) when fitted to experimental spectra with surface-conditioned cubic temperature profile ........... 90

Figure 4.7: $\mathrm{CH}_{3} \mathrm{OH}$ spectra fits to experiment using temperature profile from the present study with $T(0)=T_{\text {boiling }}$ conditioned fit with concentration forced decay to $<80 \% 91$

Figure 4.8: Predicted $\mathrm{CH}_{3} \mathrm{OH}$ mole fraction profile for two constraint cases when fitted to experimental spectra at $2800-3100 \mathrm{~cm}^{-1}(\mathrm{C}-\mathrm{H}$ stretching $)$............................... 93

Figure 4.9: $\mathrm{CH}_{3} \mathrm{OH}$ spectra fit to experiment in $\mathrm{C}-\mathrm{H}$ stretching band with two different constraint models

Figure 4.10: Modified temperature profile illustrating the assumed displacement of $T$ profile caused by stainless steel cone and $\mathrm{N}_{2}$ purge

Figure 4.11: Predicted $\mathrm{CH}_{3} \mathrm{OH}$ mole fraction profile for two constraint cases when fitted to experimental spectra with a modified temperature profile, $T_{\bmod }$ 96

Figure 4.12: $\mathrm{CH}_{3} \mathrm{OH}$ spectra fits to experiment with two different constraint models

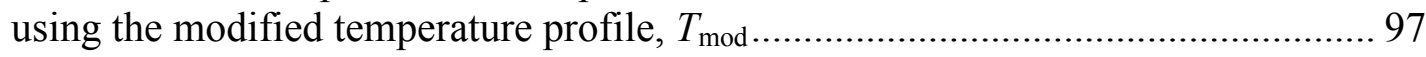

Figure 4.13: Comparison of $\mathrm{CH}_{3} \mathrm{OH}$ mole fraction fits using various values of $A_{\min } \ldots .98$

Figure 4.14: $\mathrm{CH}_{3} \mathrm{OH}$ spectra fits to experiment with $80 \%$ decay condition, $T=T_{\text {mod }}$, for $A_{\min }=0.005,0.02$.

Figure 4.15: Temperature-dependent absorption coefficients of $\mathrm{CO}$ 101

Figure 4.16: Temperature-dependent absorption coefficient for asymmetric stretching band of $\mathrm{CO}_{2}$

Figure 4.17: Constraints used by the spectral optimization solver for $\mathrm{X}_{\mathrm{CO}}$ and $\mathrm{X}_{\mathrm{CO} 2}$ profile fits

Figure 4.18: Comparison of model fits for $\mathrm{X}_{\mathrm{CO} 2}$ and $\mathrm{X}_{\mathrm{CO}}$ using $T_{\text {orig }}$ and $T_{\bmod }$ temperature profiles

Figure 4.19: $\mathrm{CO}$ and $\mathrm{CO}_{2}$ spectra fits in 2000 to $2400 \mathrm{~cm}^{-1}$ region compared to experiments through $z=15 \mathrm{~cm}$ using $T_{\text {orig. }}$

Figure 4.20: $\mathrm{CO}$ and $\mathrm{CO}_{2}$ spectra fits in 2000 to $2400 \mathrm{~cm}^{-1}$ region compared to experiments through $z=15 \mathrm{~cm}$ using $T_{\bmod }$ 108

Figure 4.21: Model fits for $\mathrm{X}_{\mathrm{CO} 2}$ and $\mathrm{X}_{\mathrm{CO}}$ when solved in independent spectral regions 
Figure 4.22: Modeled absorbance spectra fits for $\mathrm{CO}_{2}$ and $\mathrm{CO}$ bands when solved independently ................................................................................... 110

Figure 4.23: A comparison of model fits for $\mathrm{X}_{\mathrm{CO}}$ using two different values of $A_{\min } . .112$

Figure 4.24: Modeled absorbance spectra fit for $\mathrm{CO}$ band using $A_{\min }=0.01,0.005 \ldots 113$ 


\section{CHAPTER 1: Introduction}

Pool fires are buoyancy-driven diffusion flames fed by the vaporization of a horizontally aligned, condensed-phase fuel. Volatile liquids and solids can serve as fuels for pool fires. The characteristic length of a pool fire is the pool diameter, $d$. This was justified by Blinov and Khudiakov, who demonstrated that burning rates of pool fires are a function of $d$, which determines the surface area of the fuel exposed to oxygen. ${ }^{1}$ The sizes of pool fires span several orders of magnitude, from matches $\left(10^{-3} \mathrm{~m}\right)$ to fuel spills $\left(10^{2} \mathrm{~m}\right) . \quad d$ is used as the characteristic length in the definition of non-dimensional numbers describing flame structure, such as the Froude number ${ }^{2}$ and the Strouhal number. ${ }^{3}$ The structure and behavior of the fires vary greatly depending on the fuel properties and the pool diameter.

\subsection{Heat Transfer in Pool Fires}

Heat transfer is very important in the characterization of pool fires. A large fraction of the heat created by combustion is transferred to the fuel surface, causing fuel vaporization. The vaporized fuel is transported through a fuel-rich zone to the reacting flame region. The "positive feedback loop" ${ }^{4}$ between the flame heat generation and fuel vaporization largely determines the pool fire size and structure. Accurate modeling of the heat transfer in this process is key to understanding pool fire dynamics. Three parameters

important to the role of heat feedback are the combustion heat release rate, $\dot{Q}_{c}$, the heat 
rate required for fuel vaporization, $\dot{Q}_{v a p}$, and the ratio between the two, $\chi_{s}$, as defined by Hamins et al. ${ }^{4}$ :

$$
\chi_{s}=\frac{\dot{Q}_{v a p}}{\dot{Q}_{c}}
$$

$\dot{Q}_{c}$ and $\dot{Q}_{v a p}$ are given by:

$$
\begin{gathered}
\dot{Q}_{c}=\dot{m} H_{c} \\
\dot{Q}_{v a p}=\dot{m} \cdot\left[H_{v a p}+\int_{T_{0}}^{T_{s}} c_{p} d T\right]
\end{gathered}
$$

where $\dot{m}$ is the fuel burning rate, $H_{c}$ is the ideal heat of combustion, $H_{v a p}$ is the heat of vaporization, $c_{p}$ is the temperature-dependent specific heat of the fuel with integration limits of $T_{s}$, the surface temperature, and $T_{o}$, the ambient temperature. ${ }^{5} \chi_{s}$ represents the fraction of $\dot{Q}_{c}$ needed to vaporize the fuel, and is related to the inverse of the diffusive transfer number of the fuel, as first designated by Spalding, ${ }^{6}$ :

$$
B=\frac{H_{c}}{H_{v a p}}
$$

A fraction of $\dot{Q}_{c}$ is transferred to the fuel surface by conduction, convection, and radiation. A considerable amount of work exists to quantify the fraction of heat carried by each mode. Hottel $^{7}$ provides the following equations for heat transfer to the fuel surface by each mode in terms of flame properties:

$$
\begin{gathered}
\dot{Q}_{s, \text { cond }}=k \pi d\left(T_{f}-T_{s}\right) \\
\dot{Q}_{s, \text { conv }}=h \frac{\pi d^{2}}{4}\left(T_{f}-T_{s}\right)
\end{gathered}
$$




$$
\dot{Q}_{s, r}=\sigma V \frac{\pi d^{2}}{4}\left(T_{f}^{4}-T_{s}^{4}\right)[1-\exp (-\kappa d)]
$$

where $k$ is effective thermal conductivity, $T_{f}$ is flame temperature, $h$ is the effective heat transfer coefficient, $\sigma$ is the Stefan-Boltzmann constant, $V$ is a dimensionless configuration factor, and $\kappa$ is an extinction coefficient. These equations show as $d$ increases, the proportion of conduction decreases as convection and radiation increase. Experimental confirmation of this effect was identified Burgess and Hertzberg, ${ }^{8}$ who presented a correlation of mass flux, $\dot{m}^{\prime \prime}$, to $B$ as a function of $d$. They cited the gasoline and diesel pool fires experiments by Blinov and Khudyakov ${ }^{1}$ to show that $\dot{m}^{\prime \prime}$ is highly dependent upon $d$, and explained the phenomenon by identifying "regions" of $d$ with differing dominant modes of heat transfer to the pool surface, $\dot{Q}_{s}$. Hamins et al. ${ }^{5}$ also conducted a review of pool fire experiments to show the relationship between the ratio of $\left(\dot{m}^{\prime \prime} / B\right)$ as a function of $d$, as shown in Figure 1.1. Large, radiation-dominated fires are of greatest interest to fire safety scientists because of their potential for damage. 


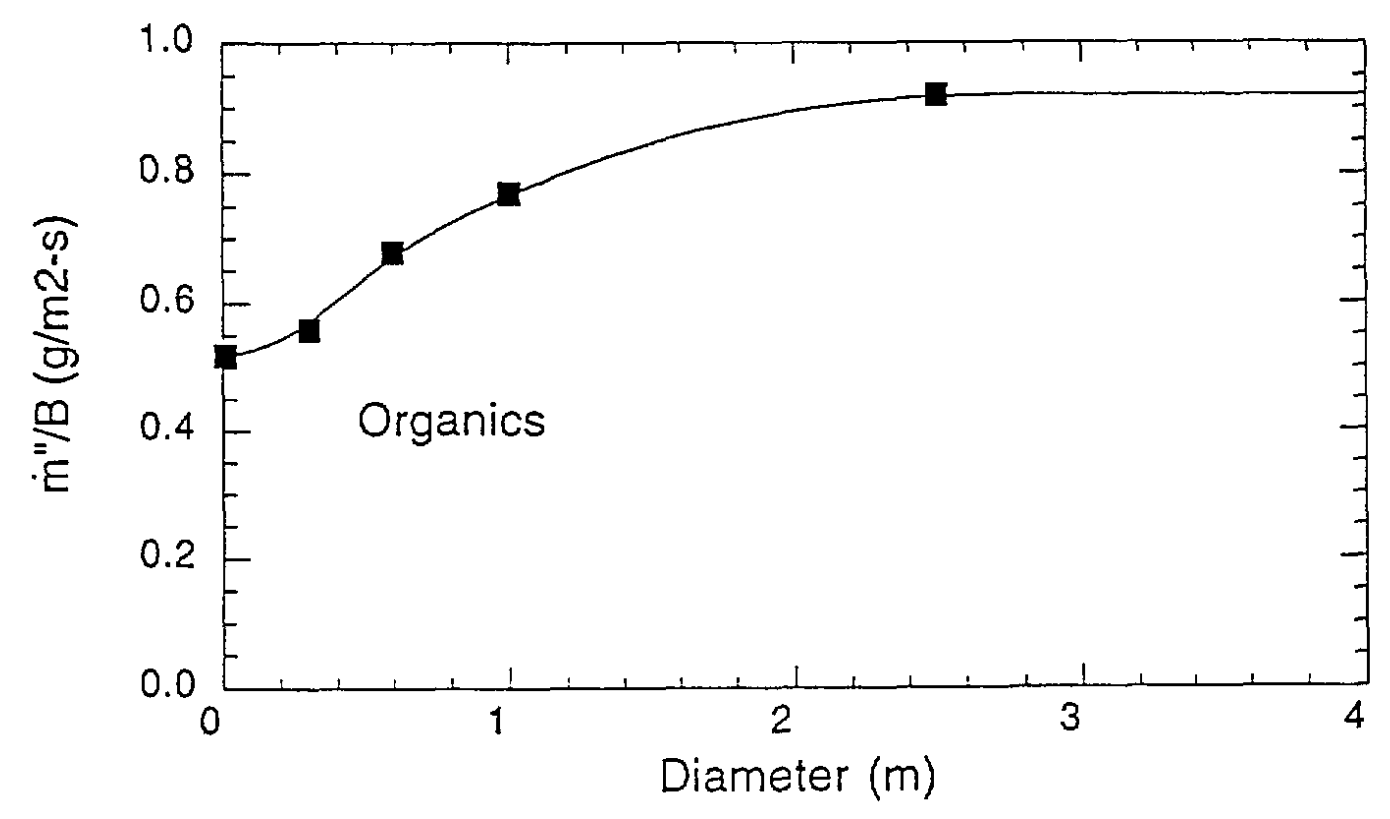

Figure 1.1: Ratio of mass flux $\dot{m}^{\prime \prime}$ to the diffusive transfer number $B$ as a function of pool diameter, reproduced from Figure 12 of Hamins et al. ${ }^{5}$ (not subject to copyright)

The heat transfer characteristics of pool fires are highly dependent on fuel properties. This is in large part because the gas separating the flame from the pool surface - the so-called "fuel-rich core" - is dominated by fuel vapor and products of fuel vapor pyrolysis. The convective heat transfer rate to the surface $\dot{Q}_{s, \text { conv }}$ is dependent upon the molecular diffusivity of the fuel and flame gases, and the radiative heating rate at the surface $\dot{Q}_{s, r}$ depends upon soot content and the radiative properties of the gases in the fuel-rich core. For sooty flames, soot radiates like a blackbody and increases the emissivity in flames, and graybody radiation can be used in modeling emission of sooty fires. ${ }^{9}$ In contrast, low-soot flames emit spectral radiation and graybody models tend to overestimate emission. ${ }^{9}$ The spectral radiation properties of combustion products, such as $\mathrm{CO}_{2}, \mathrm{H}_{2} \mathrm{O}$ and $\mathrm{CO}$ have been the subject of several studies ${ }^{9-11}$ and are documented 
thoroughly in databases such as HITRAN and HITEMP. ${ }^{12}$ Because radiation is the dominant form of heat transfer in large fires, its characterization is critical for fire safety science.

The value of $\dot{Q}_{s, r}$ is a function of the total heat emitted, $\dot{Q}_{r}$. The fraction of combustion energy released by thermal radiation is given by:

$$
\chi_{r}=\frac{\dot{Q}_{r}}{\dot{Q}_{c}}=\frac{\dot{Q}_{r}}{\dot{m} H_{c}}
$$

Hamins et al. ${ }^{5}$ note that for $d<2 \mathrm{~m}, \chi_{r}$ depends only upon the type of fuel; for $d>2 \mathrm{~m}$, $\chi_{r}$ becomes $d$ dependent as well. They describe two methods used by researchers to measure values of $\dot{Q}_{r}$. One is a heat flux distribution integral method, where radiometers are used over a defined boundary to measure heat flux and then integrate flux over area. The second method uses a distant point measurement of heat flux and using an isotropic radiation assumption to integrate the point measurement over an assumed spherical boundary. Once $\dot{Q}_{r}$ is measured, the value of $\chi_{r}$ can be calculated by using measurements of $\dot{m}$ to find the total heat release rate. The value of $\dot{Q}_{s, r}$ is also a function of flame geometry, flame temperature, soot fraction, and the effects of vaporized fuel and pyrolized intermediate fuel species inside the fire. Given the complexities of turbulent flame kinetics and mass and heat transfer in three-dimensional nonhomogenous space, solutions for $\dot{Q}_{v a p}$ via conservation equations are typically formed with empirical or semi-empirical methods. ${ }^{5}$ Geometric calculations dependent on flame shape and size were simplified by assuming a cylindrical flame shaped ${ }^{13}$ and also a flame shape defined by a polynomial. ${ }^{14}$ De Ris ${ }^{15}$ developed a simplified radiation model for 
pool fires to further simplify the calculations. The model identifies an effective radiation temperature, flame shape and size, and a flame absorption-emission coefficient, $\kappa$. De Ris showed that $\kappa$ is the most sensitive parameter of all. Citing previous work ${ }^{9,16}$ demonstrating the limitations of gray, homogeneous models for large $(d>50 \mathrm{~cm})$ and low-soot fuels, Brosmer and Tien ${ }^{10}$ developed a two-layer flame model which included a hot, luminous flame and a cool, fuel-rich core. This model used a two-part mean beam path to calculate radiation flux in PMMA fires with 1 percent error in $d=38 \mathrm{~cm}$ fires and 10 percent error in $d=73 \mathrm{~cm}$ fires. The increasing error for large diameter was due in part to the proportional increase in size of the cool, fuel-rich core. Brosmer and Tien's model used gray, isothermal absorption data for the fuel-rich core, thereby not capturing some important features of the fuel-rich core structure as it relates to radiative heat transfer. Wakatsuki ${ }^{17}$ developed an experimental database of temperature-dependent spectral absorption coefficients, $\kappa_{v}$, for vaporized fuels. The purpose of this database was to provide data to evaluate spectral radiative transfer through the various temperature zones of a pool fire fuel-rich core.

Several researchers have evaluated radiative heat transfer as it relates specifically to methanol pool fires. Methanol pool fires are non-sooting, and therefore heat transfer to the surface via radiation is less dominant relative to other fuels. Akita and Yumoto ${ }^{18}$ conducted experimental measurements of methanol fires in cylindrical ring burners and observed high mass flux on the outer rings. They concluded that radiative heat transfer was negligible when compared to conduction and convection; however, this finding has since been questioned. Hamins et al. ${ }^{4}$ showed that for $d=30 \mathrm{~cm}$ methanol, heptane and toluene pool fires, a majority of the heat flux to the surface is used to heat and vaporize 
the fuel consumed during combustion, with relatively small heat losses due to surface reflection, re-radiation, and loss to surroundings. They also used a heat balance equation to experimentally determine that the radiative fraction of heat flux to the fuel surface for toluene, heptane, and methanol pool fires were $96 \%, 80 \%$ and $53-55 \%$, respectively. These results showed that radiation is still the dominant heat flux in non-sooty fires of moderate pool diameter. In the case of methanol, Hamins et al. also showed that as fuel lip height increased, overall mass flux decreased. Also, changes in the fuel lip height created qualitative differences in the mass flux as a function of pan radius, suggesting the importance of convection near the burner rim.

Computer models of radiation have also been tested by a number of researchers. Hostikka et al. ${ }^{19}$ modeled radiation transfer in NIST's Fire Dynamics Simulator (FDS) using a Large Eddy Simulation (LES) and a wide-band model for a variety of methanol pool fire geometries. These models used $\mathrm{CH}_{4}$ absorption coefficients for the fuel vapor due to the lack of adequate data at the time on high-temperature methanol absorption coefficients. The study concluded that the model was susceptible to large quantitative errors due to inaccuracies in gas phase temperatures. Wakatsuki et al. ${ }^{20}$ postulated that this might be due to the fact that the temperature-dependent absorption coefficients of the vaporized methanol was not properly accounted for. They used their measured methanol absorption coefficients to evaluate heat transfer along the centerline of a $d=30 \mathrm{~cm}$ methanol pool fire using a 1-D radiative transport model and species concentration and temperature data obtained from a NIST Fire Dynamics Simulator (FDS) simulation. Their calculation assumed background radiation from a $1400 \mathrm{~K}$ flame at a height of $0.74 \mathrm{~m}$ above the pool surface. Using temperature-dependent values of $\kappa_{v}$ for methanol, their 
prediction of emissive intensity, $I$, at the fuel surface center was within 2 percent of the experimental measurements by Hamins et al. ${ }^{4}$

Several others have also measured the temperature and behavior of methanol

fires. Weckman and Strong ${ }^{21}$ studied a $d=31 \mathrm{~cm}$ methanol pool fire using fine wire thermocouples and laser Doppler anemometry to measure temperature and velocity fields inside the fire. With time-resolved instrumentation, their measurements were also able to quantify the transient behavior of the flame and its plume. Gorchkov and Hamins ${ }^{22}$ also provide radiation-corrected temperature measurements inside methanol pool fires of $d=$ $10,30 \mathrm{~cm}$ with a time response of $0.1 \mathrm{~s}$ and an uncertainty $(k=1)$ of $\pm 50 \mathrm{~K}$. This data was used by Hostikka et al. ${ }^{19}$ for evaluation of the radiation model in FDS. With the abundance of experimental data and numerical models for methanol pool fires at $d=30$ $\mathrm{cm}$, this fire is a good starting point for any experiment designed to study non-sooty pool fires where a majority of heat transfer to the fuel surface is via radiation.

\subsection{Radiative Transfer Equations}

To more fully understand the mechanisms for radiation heat transfer in flames, it is good to review the basics of radiation heat transfer. C.L. Tien's seminal publication entitled "Thermal Radiation Properties of Gases" the fundamental quantities used to describe electromagnetic radiation from the photon level to total heat flux calculations and also gives a detailed explanation of the quantum effects associated with the interactions between photons and molecules. This section will largely be an abridged summary of the explanations by Tien as relevant to the study 
presented in this thesis, including a clarification of some definitions as to how they are used in the treatment of experimental data.

Electromagnetic radiation refers to the energy carried by photons and the electric and magnetic fields they create during their motion. The energy carried by a single photon is:

$$
e=h v
$$

where $v$ is photon frequency, and $h$ is Planck's constant. The photon distribution function, $f_{v}$, describes the number of photons in the frequency interval $v+d v$, moving in the solid angle element $d \Omega$ about the direction $\mathbf{s}: f_{v}(\mathbf{r}, \mathbf{s}, t) d \Omega d v$. Specific intensity, $I_{v}$, describes the energy in the photons and is more applicable to physical problems. It is related to $f_{v}$ by:

$$
I_{v}=\operatorname{chvf_{v}}
$$

where $c$ is the speed of light. All spectral variables can be converted into total values by integrating over the entire spectrum, e.g. total intensity, $I$, is defined as:

$$
I \equiv \int_{0}^{\infty} I_{\nu} d v
$$

Spectral radiant energy flux $q_{v}$ is the radiant energy across a surface aligned arbitrarily across a coordinate axis. Where $\mathbf{n}$ is the unit vector normal to the chosen surface,

$$
q_{v}=\int_{0}^{4 \pi} I_{v}(\mathbf{n} \cdot \mathbf{s}) d \Omega
$$

This is integrated over the entire sphere, $\Omega=4 \pi$ steradian (ster). For hemispherical radiance, the integral is over $\Omega=2 \pi$ ster. Total heat flux $\dot{Q}$ is derived from eq. (1.10), 
though there are engineering estimates that fire scientists have used for pool fires, as described earlier in the section.

\subsubsection{Fundamentals of Spectral Radiation}

In pool fires, radiation emitted by the flame and directed at the fuel surface invariably passes through the "fuel-rich core," which is composed mainly of fuel vapor and intermediary combustion species. These compounds absorb and emit radiation spectrally. It is important to understand the spectral absorbance properties of the species inside the "fuel-rich core" in order to calculate their affect on radiation to the fuel surface. This section describes how the carriers of radiative energy, photons, interact with molecules and explains the parameters that influence this interaction.

Photons carry a quantified amount of energy, $e$, directly proportional to their frequency, v. Photons are absorbed and emitted by atoms and molecules only when it can cause a quantized change in energy state. For instance, to excite a bound electron from ground state (0) to the first quantum energy state (1), it requires the absorption of a photon carrying energy equal to the difference of the energy states:

$$
\begin{gathered}
e_{1}-e_{0}=\Delta e=h v \\
v=\frac{\Delta e}{h}
\end{gathered}
$$

The type of energy transitions occurring in the molecules determines the frequency of the interacting photon. There are three types of energy transitions: (1) transitions within atomic or molecular states are called bound-bound, (2) transitions 
between an atomic or molecular state to a free state, such as electron ionization or molecular dissociation, are called bound-free, (3) a transition of energy between free energy carriers are called free-free. Bound-free and free-free interactions can change to any energy states, and their interactions emit or absorb photons in a wide range of energies. Conversely, bound-bound interactions involve interactions between quantized energy states of matter. These interactions involve changes in electron energy levels, and in molecules, also involve changes in vibrational and rotational energy states. Boundbound transitions are the most common in infrared radiation, such as with pool fire environments, with bound-free and free-free transitions occurring only under ionizing conditions $(>6000 \mathrm{~K}) .^{23}$ The three types of bound-bound transitions (electronic, vibrational, and rotational) occur at different energy levels. Electronic energy changes create absorption-emission lines in the ultraviolet, visible, and near infrared wavelengths $\left(10^{-2} \mu \mathrm{m}<\lambda<1.5 \mu \mathrm{m}\right)$, vibrational absorption-emission lines are typically in the middle infrared range $(1.5 \mu \mathrm{m}<\lambda<20 \mu \mathrm{m})$, and rotational absorption-emission lines are in the far infrared $(20 \mu \mathrm{m}<\lambda<1000 \mu \mathrm{m})$. In the temperatures of flame environments, interactions in the mid-IR range, i.e. vibrational energy transitions, have the greatest impact on radiative heat transfer. Electronic transitions occur at much higher photon frequencies than typically found in flames and rotational transitions occur at photon frequencies which are too low. Electronic transitions can be ignored, but rotational transitions often occur concurrently with vibrational transitions, so both will be discussed in further detail.

It is important to note that photons interact in rotational and vibrational energy transitions only in molecules where the energy transition changes the molecule's dipole 
moment. This means that atomic and homonuclear diatomic gases do not exhibit any infrared absorption spectra, which limits the compounds that can be measured, but also allows an experimenter to eliminate unwanted absorption by purging with the noninteracting gases (e.g. Ar, $\mathrm{N}_{2}$ ). While linear triatomic molecules (e.g. $\mathrm{CO}_{2}$ ) will not change their dipole moments with symmetric vibration, asymmetric vibration will change their dipole moments, and thus will exhibit infrared absorption.

Energy states on an atomic scale can be described with the same models used in general mass physics with the addition of quantum mechanics principles. Molecular rotational energy is described by a "rigid-rotator model" describing rotational energy levels, which in a diatomic molecule is:

$$
\varepsilon_{j}=h c_{0} B j(j+1) \quad j=0,1,2 \ldots
$$

where $B \equiv h /\left(8 \pi^{2} c_{0} t\right), j$ is the angular-momentum quantum number, and $t$ is the molecular moment of inertia. Quantum selection rules state that energy changes can only occur for $\Delta j= \pm 1,0$. Typically, a straight change in rotational energy is very low energy (far-IR) and is not important in combustion environments. However, rotation still plays a role in vibrational energy changes, which are far more important to flame studies.

Vibrational energy states, $\mathcal{E}_{v}$, can be conceptualized by an oscillating spring model where the "spring force" is the Coulombic force between the atoms, which has an equilibrium interatomic distance. A harmonic oscillator assumption is made for the vibrating model, where the amplitude of oscillation is related to the energy state: 


$$
\varepsilon_{v}=h v_{v}\left(v+\frac{1}{2}\right) \quad v=0,1,2 \ldots
$$

where $v$ is the vibrational quantum number and $v_{v}$ is the frequency of oscillation. Molecular vibration may have several modes, depending upon the structure of the molecule. For instance, diatomic molecules (e.g. $\mathrm{CO}$ ) have only bond stretching, while triatomic molecules (e.g. $\mathrm{CO}_{2}$ ) may have symmetric and asymmetric stretching, as well as bending. More complex molecules have different types bonds and more modes of vibration, which would create a more complex absorption-emission spectrum. In a harmonic oscillator, quantum selection rules are $\Delta v= \pm 1$, which is called the "fundamental" transition. Real molecules act as anharmonic oscillators, which introduces non-linearity into the energy equation, allowing the quantum transitions $\Delta v= \pm 2, \pm 3$, etc. These transitions are called "harmonics" or "overtones," where $\Delta \varepsilon_{v}$ of overtone transitions are approximately integer multiples of $\Delta \varepsilon_{v}$ for the fundamental transition. A schematic of the anharmonic model with a fundamental transition and a " 1 st overtone" is shown in Figure 1.2, where the magnitudes of the arrows show the relative values of $\Delta \varepsilon$ for each transition. 


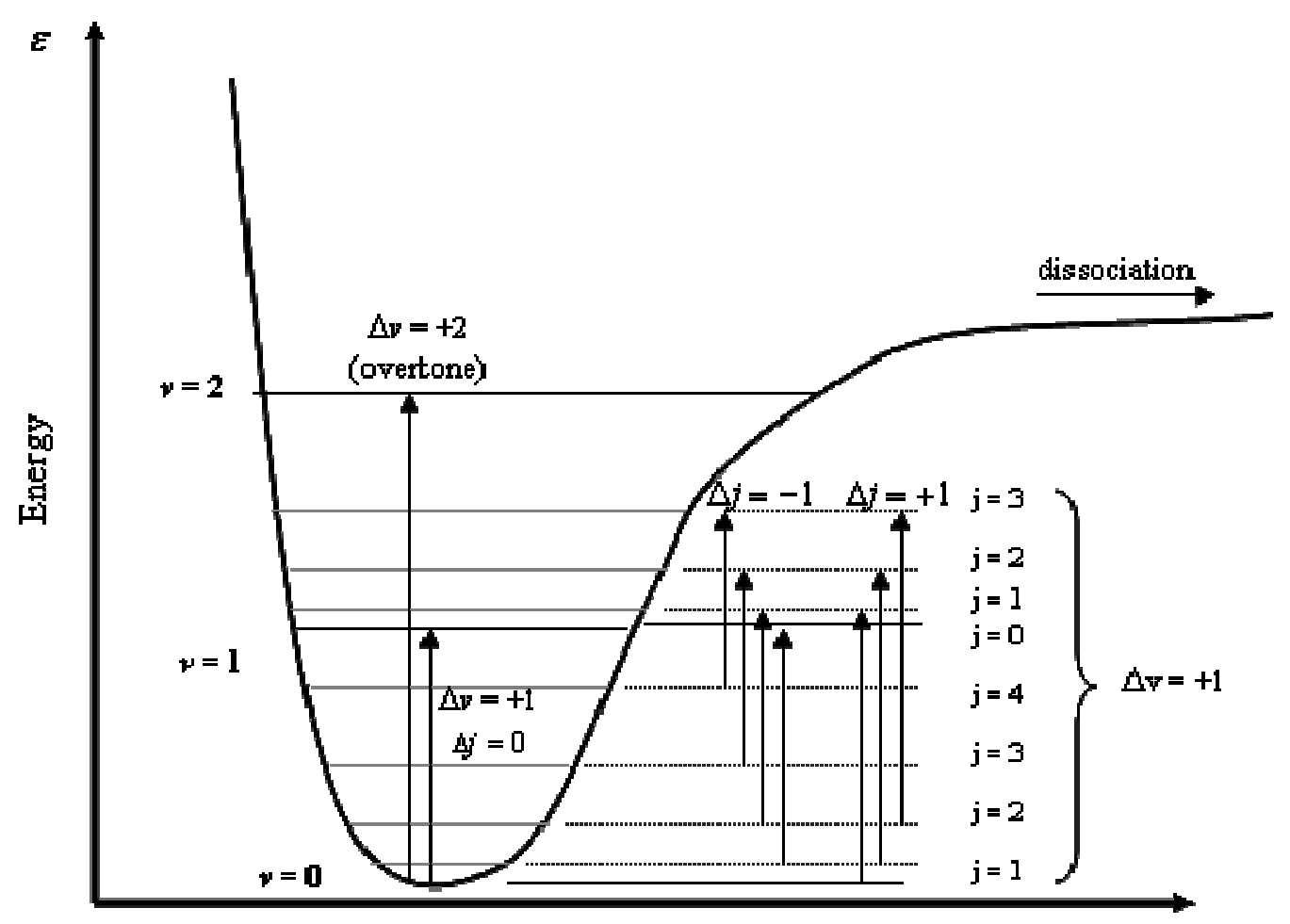

Interatomic distance

Figure 1.2: Vibrating-rotator model of a molecule illustrating vibrational $(v)$ and rotational $(j)$ energy levels and transitions (adapted from figures by Tien, ${ }^{11}$ Brewster, ${ }^{23}$ and Wakatsuki ${ }^{17}$ )

The photon frequencies measured in FT-IR (mid-IR) spectrometry are centered about vibrational energy states. These energy changes are often combined with the much smaller rotational energy changes, resulting in absorption behavior called a vibratingrotator. The vibrating-rotator model for a $\Delta v=+1$ transition is shown on the right-side of Figure 1.2. The model predicts the combined effect of quantum changes in $v$ with possible rotational energy state changes $(\Delta j=0, \pm 1)$. During a $\Delta v=+1$ transition, the energy required for a $\Delta j=+1$ transition is greater than that required for $\Delta j=0$, which is greater than that required for $\Delta j=-1$. Importantly, the initial value of $j$ affects the magnitude of $\Delta \varepsilon$ during $\Delta v=+1, \Delta j= \pm 1$ transitions: the centrifugal forces from rotation 
causes an increase in interatomic distance, which creates a larger moment of inertia, $t$, and therefore, larger changes in energy. Molecules in excited rotational states can undergo a $\Delta v=+1$ transition that requires greater photon energy to change with $\Delta j=+1$ and lesser photon energy to change with $\Delta j=-1$. This difference in $\Delta \varepsilon$ is shown by the length of the arrows in Figure 1.2, which is dependent upon initial $j$. Peaks that result in $\Delta \varepsilon$ increase because of $\Delta j=+1$ transition are populated in the "R-wing" and the peaks that result in $\Delta \varepsilon$ decrease because of $\Delta j=-1$ transition are populated in the "P-wing". The $\Delta j=0$ peaks populate "Q", and overlap along the central peak, $\omega_{0}$. All of these effects create a distribution of peaks in absorption-emission bands, as shown in Figure 1.3 .

As the temperature of the spectrally absorbing medium increases, several important temperature effects occur. First, the population of molecules at excited states of vibration and rotation increases with a distribution characterized by the Boltzmann distribution, which "expands" the $\mathrm{P}$ and $\mathrm{R}$ wings of absorption-emission, and reduces the Q peak. Second, increased temperature makes molecules less likely to participate in absorption. Third, high temperatures and/or pressures create more collisions, which causes small, instantaneous changes in the energy state of the molecule, allowing for a slightly larger range of $v$ to interact with these transitions. This effect is called "collision broadening" because it creates broadening among the absorption-emission lines, decreasing the height of the central peak and broadening it across a larger bandwidth. More frequent (high $P$, high $T$ ) collisions amplify this effect. Other numerous broadening effects, e.g. Doppler broadening, also play a role at various temperatures. All of these processes make infrared absorption dependent upon temperature. 


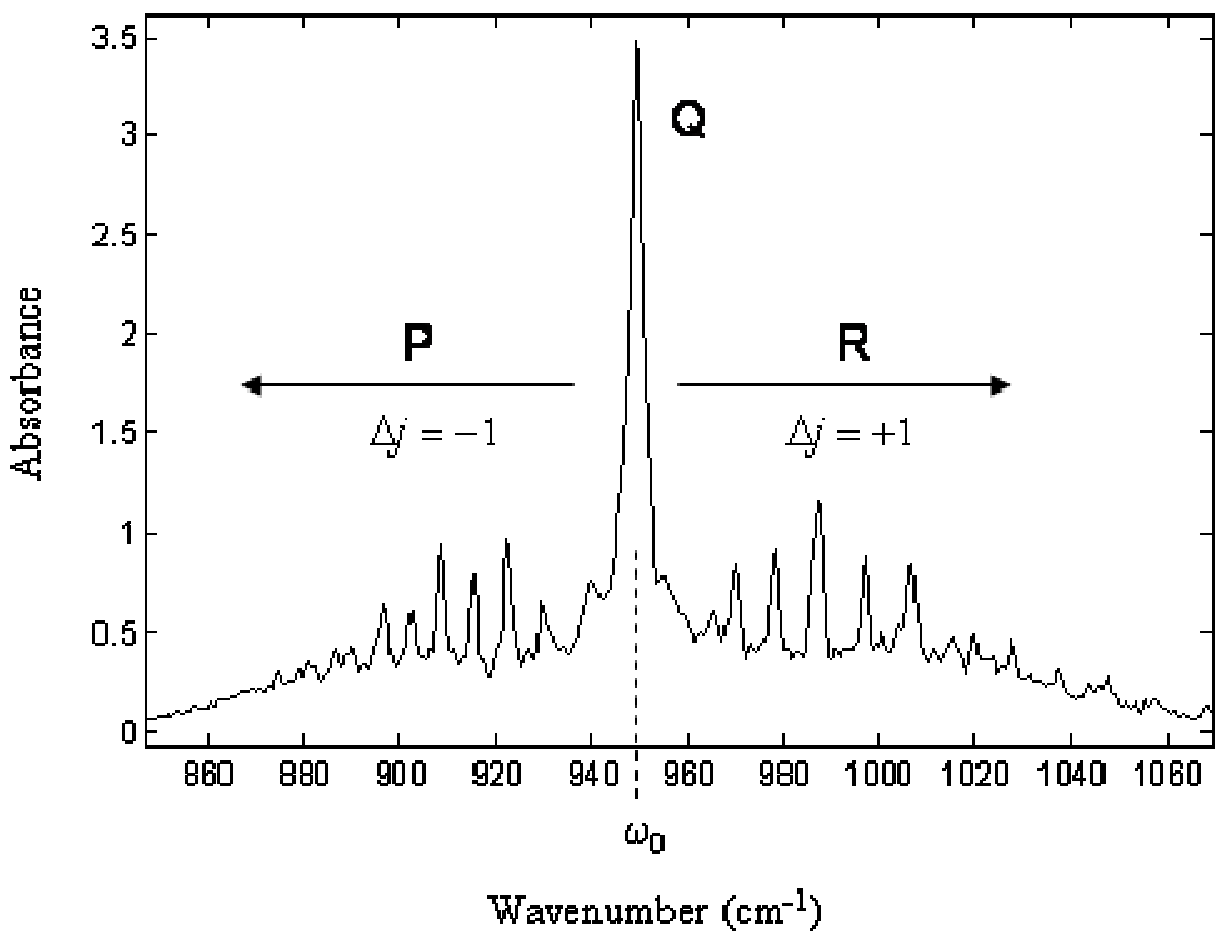

Figure 1.3: Absorbance peak of $\mathrm{C}_{2} \mathrm{H}_{4}$ exhibiting energy transitions of a vibrating-rotator defined by central peak, $\omega_{0}$, and $\mathrm{P}, \mathrm{Q}$, and $\mathrm{R}$ wings

\subsubsection{Definition of absorption coefficients}

In spectroscopy, the spectral intensity is often directly or indirectly measured. The equation of transfer for a particular frequency along a line-of-sight measurement of a non-scattering medium is:

$$
\frac{d I_{v}}{d z}=-\kappa_{v}\left(I_{v}-I_{b v}\right)
$$

Eq. (1.16) is derived by using a photon conservation equation for a finite volume, where $I_{b v}$ is local blackbody intensity at frequency $v, \kappa_{v}$ is the spectral volumetric absorption coefficient of the medium, and $z$ is optical pathlength. The equation shows that the 
amount of attenuation is related to the intensity, and $\kappa_{\nu}$ describes the "fractional reduction of intensity per unit length."11 Emission is related to the blackbody intensity, $I_{b}$, which is described by the Planck blackbody function:

$$
I_{b v}=\frac{2 h v^{3}}{c^{2}\left(\exp \left[\frac{h v}{k_{B} T}\right]-1\right)}
$$

where $T$ is the temperature of the medium, $k_{B}$ is Boltzmann's Constant, and $I_{b v}$ is the blackbody intensity as a function of frequency. For an optical pathlength $z$ in a gaseous medium, the Beer-Lambert Law describes spectral transmissivity, $\tau_{v}$ :

$$
\tau_{v}(z) \equiv \frac{I_{v}(z)}{I_{v}(0)}=\exp \left(-\int_{0}^{z} \kappa_{v} d z\right)
$$

The term calculated by the integral in eq. (1.18) is also called the optical depth. The total absorption coefficient of the medium is the sum of the partial absorption coefficients in the medium:

$$
\kappa_{v}=\sum_{k} \kappa_{v, k}
$$

It follows from exponent and integral rules and eqs. (1.18) and (1.19) that:

$$
\tau_{v}=\prod_{k} \tau_{v, k}
$$

For the purposes of using an absorption coefficients database which is not specific to concentration, a new spectral absorption coefficient may be defined as:

$$
\kappa_{v, k}^{\prime}=\frac{\kappa_{v, k}}{P_{k}}
$$

where $P_{k}$ is the concentration of the medium expressed in partial pressure. The new form of eq. (1.19) for use in Beer-Lambert becomes: 


$$
\kappa_{v}=\sum_{k} \kappa_{v, k}^{\prime} P_{k}
$$

Absorbance, $A$, is defined as:

$$
A_{v}(z)=-\ln \left(\frac{I_{v}(z)}{I_{v}(0)}\right)=-\ln \left(\tau_{v}\right)
$$

Note that this definition is different from the definition of absorptivity, $\alpha_{v}$, which is the fraction of energy being absorbed, where in a non-scattering, non-reflecting medium:

$$
\alpha_{v}=1-\tau_{v}
$$

The main motivation behind using absorbance is because measurements in units of $A$ are directly proportional to species concentration, for constant $z$. For a homogeneous pathlength $z$, eq. (1.23) may be simplified to the form:

$$
\begin{gathered}
A_{v, k}=\kappa_{v, k}^{\prime} P_{k} z \\
A_{v}=\sum_{k} A_{v, k}=\sum_{k} \kappa_{v, k}^{\prime} P_{k} z
\end{gathered}
$$

Absorbance $A_{v}$ is a measurement provided by an FT-IR experiment, which is explained in greater detail in Chapter 3. With a database of $\kappa_{v}^{\prime}$ for various reactant and combustion species $k$ and experimental control of pathlength $z$, it is possible to design an FT-IR experiment to measure species concentrations $P_{k}$. The amount of information one can gain is dependent upon the experiment's design, which is discussed in Chapter 2.

\subsection{Use of spectroscopy in combustion studies}

Fourier Transform Infrared (FT-IR) spectroscopy is a method that has been used to analyze combustion. An FT-IR spectrometer uses a Michelson interferometer setup 
with one fixed mirror and one moving mirror to create an interferogram of signal strength as a function of mirror displacement (distance domain). A Fourier transform converts the interferogram in a spectrum (frequency domain). One example of its use is to measure spectral intensity, which can be used with eq. (1.17) to calculate flame temperature, $T_{f}$. The emitted radiation spectrum is also useful as a boundary condition for the study of heat transfer from a flame. Localized temperature measurements are more complicated. With the Planck blackbody function and the HITRAN database, Clausen $^{24}$ used an FT-IR probe to measure local temperatures within a coal flame with prior knowledge of gas concentrations. By subtracting background radiation from each subsequent measurement, a tomography of temperatures were obtained that were in good agreement with pyrometer results. However, the use of a cooled probe in a combustion zone did cause some temperature perturbations that affected results. The experiment showed the viability of using probes in full-scale combustion environments for FT-IR spectroscopy.

FT-IR has also been used to study concentrations of high-temperature species within an assortment of combustion systems, including staged burners, ${ }^{25}$ flue gases, ${ }^{26}$ and diffusion flames. ${ }^{27,} 28$ Martin et al. $^{27}$ used absorption spectra and transmission spectroscopy to study the concentrations of $\mathrm{CO}$ and $\mathrm{CO}_{2}$ in black liquor char combustion. Their study showed sensitivity to purging rates for their probe, which was also the air supply for their reactor. Best et al. ${ }^{28}$ used transmission and emission FT-IR tomography to study the chemical structure of a sooty laminar diffusion flame. The experiment consisted of horizontal line-of-sight measurements through numerous regions of the flame and used an Abel conversion to transform the measurements into topographical data. Their data provided cross-sections of temperature and relative concentrations of 
$\mathrm{CO}_{2}, \mathrm{H}_{2} \mathrm{O}$, soot, alkanes, alkenes, and alkynes in an ethylene flame, which were in good agreement with previous studies.

FT-IR spectroscopy is beneficial for in situ concentration measurements, whereas other methods (e.g. gas chromatography) may allow further reactions to occur before samples can be measured. In this sense, it is a logical tool to study quickly reacting combustion environments, such as fuel decomposition. However, because of the lack of high-temperature spectral absorption information for fuel species, there are not many, if any, publications pertaining to this type of study which provide values for absolute concentrations.

\subsection{Summary of thesis}

The goal of this thesis is to provide an experimental set of data for validating nonsooting pool fire radiation transport models. The temperature and species mole fractions within the "fuel-rich core" are the main consideration in this study. NIST's Fire Dynamics Simulator (FDS) uses a simplified reaction chemistry model to reduce computational demands, but the simplified chemistry does not provide adequate details to predict local gas temperatures and mixture fractions in the fuel rich core. Because it is sensitive to temperature and species profiles in the fuel rich core, radiation heat transfer to the pool surface and related fuel-mass burn rates in pool fires can not be accurately simulated by current FDS modeling tools.

An in situ experiment was created to directly measure the influence of fuel compounds on radiation by recording the spectrum of a blackbody infrared source as it passes vertically through the centerline of a $d=0.3 \mathrm{~m}$ methanol pool fire. Wakatsuki's 
database of temperature-dependent absorption coefficients facilitates the interpretation of absorption spectra through the fuel rich cores in terms of species concentrations and temperatures. The results give insight on the decomposition of fuel and production of combustion byproducts through various regions of the fuel-rich core. A radiative transport model can compare these results with radiation transport predicted by species and temperature profiles from FDS. The comparison will allow fire modelers to assess the quality of fire codes and help improve them. In the remainder of this thesis, the development of an experimental method for the measurement of major species concentrations in a $d=0.3 \mathrm{~m}$ methanol pool fire and its impact of on radiative heat transfer will be presented. Each chapter discusses major topics in the development of the experiment or the results of the study, as outlined below.

Chapter 2 will discuss the setup of the pool fire test rig. It provides a description of elements available before work on this thesis and apparatus that had to be manufactured for the experiment. Measurements required for proper analysis of pool fires are discussed, including the measurement of infrared spectra, fuel burn rates, temperature profiles, and species concentration profiles. Methods and equipment used for each measurement are discussed in detail and the experimental method is presented.

Chapter 3 presents raw data from the pool fire experiments. The infrared spectra are presented as intensity as a function of height. These intensity spectra are processed into transmission and absorbance spectra. A post-processor used to correct for extraneous gray attenuation in the absorbance spectra is also discussed. The results of temperature measurements, fuel burn rates, and concentration measurements are also presented. 
Chapter 4 will describe and present the results of a MATLAB code developed to simulate the radiative transfer through a fire. The optimization code uses a least-squares fit method to compare theoretical radiative transport to the experimental data and attempts to fit the data by adjusting the theoretical species concentration profiles. The results are presented and compared to other measurements and estimates of species profiles, including the measurement conducted in the current study and presented in Chapter 3. The sensitivity of the code to changes in input parameters (e.g. temperature profiles) is also presented.

Chapter 5 will conclude the thesis and present recommendations for future research to provide more extensive validation of complex pool fire models such as done with FDS. 


\section{CHAPTER 2: Pool Fire Experimental Systems}

Tests to study the species concentration profiles of a $d=30 \mathrm{~cm}$ methanol pool fire were performed at the National Institute of Standards and Technology (NIST) in Gaithersburg, MD. The tests were performed in an enclosure located in a lab under the supervision of the Fire Research Division of the Building and Fire Research Laboratory (BFRL) at NIST. A test rig was constructed that enabled FT-IR spectrometer measurements to be taken along the centerline of a pool fire at various pathlengths from the fuel surface was constructed. Other systems to acquire in situ thermocouple measurements, fuel burn rates, and cooling system performance were also arranged.

\subsection{Experimental Apparatus}

The test bed, illustrated in the schematic of Figure 2.1, was assembled to characterize the radiation transport along the centerline of the fuel-rich region of a pool fire by utilizing FT-IR spectrometry. The test rig allowed for an FT-IR beam to pass through the center of the pool fire to measure the absorbance of the combustion reactants, intermediates, and products in a specified "experimental pathlength," while at the same time not allowing the fire environment to affect the results outside of the experimental pathlength. This was accomplished by purging as much of the non-experimental pathlength of the FT-IR beam as possible with nitrogen $\left(\mathrm{N}_{2}\right)$. This task was done with probes being purged with $\mathrm{N}_{2}$ from a gas cylinder. The purge flow rates were kept constant with an array of valves and regulators, and were quantified by an array of 
rotameters. Using probes for optical measurements inside fires can be problematic because thermal expansion will affect optical alignment and infrared readings. This can be ameliorated by cooling the probes that are directly in the fire, and shielding other equipment near the fire. Several different cooling schemes and purging arrangements were used in this experiment and they will be discussed later in the chapter.

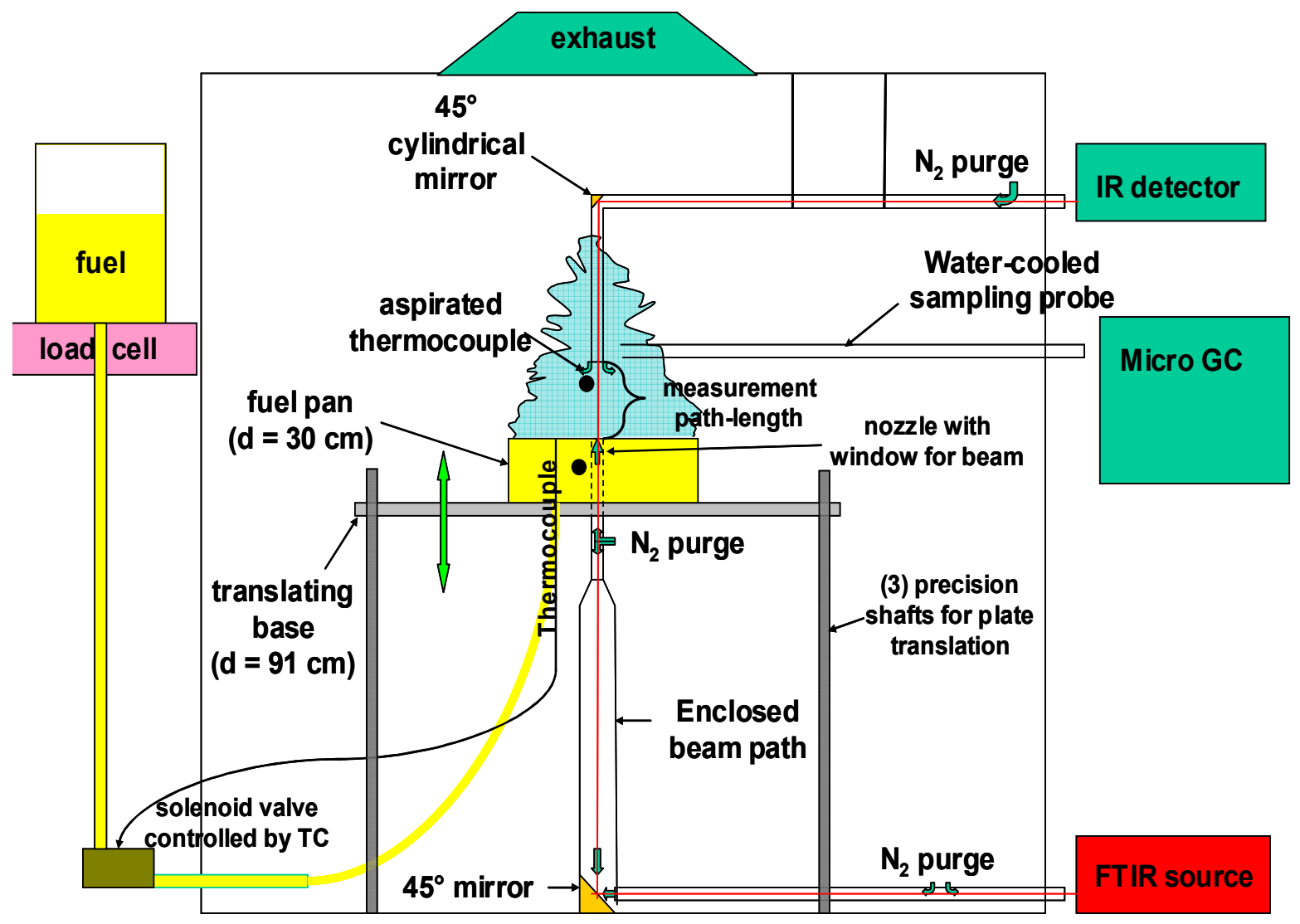

Figure 2.1: A schematic of the pool fire test rig showing the FT-IR beam path from source to detector 
The experimental pathlength is adjusted by translating the fuel pan up or down. This method was chosen, as opposed to the alternative of moving the probe and FT-IR sensor, due to the difficulty of moving and realigning the optics for every measurement. However, moving only the pan poses some challenges. First, the boundary condition for the entrainment of air changes every time the pan moves up or down. This problem was mitigated by placing the pan on a large diameter plate that limits air entrainment from directly underneath the pool level, regardless of pan elevation. Another issue is the risk of fuel spills while adjusting the plate position, which may affect measurements and poses serious safety risks. The approaches to resolve these problems are discussed later in this chapter.

This test rig measures the infrared radiation absorbance along the vertical centerline of the fire, starting from the surface of the pool up to the boundary of the probe above the fire. Measurements were taken at a spatial resolution of $5 \mathrm{~cm}$ up to $20 \mathrm{~cm}$. Each increasing height measurement includes the absorbance from the pathlengths measured before it. The results from the measured absorbance spectra had to be processed taking this into consideration, as will be discussed in a later section.

The experiment was housed inside a $2.0 \mathrm{~m}$ by $2.6 \mathrm{~m}$ room at the Building and Fire Research Laboratory (BFRL) at the National Institute of Standards and Technology (NIST). The room is enclosed on the sides and top with sheet metal, with two acrylic glass doors. The floor of the test area is cement board surrounded by a $5 \mathrm{~cm}$ high steel lip, and is there to contain damage from spilled fuel and fires. The ceiling of the enclosure is 2 meters high, with an angled inset leading to the exhaust hood. The 
ventilation system draws a maximum of 3000 CFM, with some adjustability. This facility existed in support of fire research conducted at BFRL/NIST in Gaithersburg, MD.

A $30 \mathrm{~cm}$ diameter, $7.6 \mathrm{~cm}$ deep stainless steel pan, provided by BFRL and shown in Figure 2.2, is the primary containment of methanol for the pool fire experiment. A threaded 1" stainless steel pipe fits onto a threaded 1" diameter hole in the center of the pan to allow a space for the FT-IR beam to pass through to the centerline of the flame. A cone is attached to the top of the pipe, which narrows the purged area of the hole and reduces the purge rate. A zinc selenide window epoxied onto the bottom of the cone ensures a one-way flow for the $\mathrm{N}_{2}$ purge. A more detailed description of the cone and its design iterations is included later in the chapter. The design length of the threaded hole, threaded pipe, and cone assembly is $7.6 \mathrm{~cm}$ to match the pan depth, though this is adjustable via turns in the threaded connection. A second hole on the bottom of the pan serves two purposes: first, it allows for the insertion of a sheathed thermocouple, second, the hole is large enough to provide space for the flow of fuel around the thermocouple and into the pan. Both connections are made to the pan with a reducing tee union, with the thermocouple going straight into the pan through the end of the tee with the reduced size. A fuel line attached to the other tee connection then feeds fuel around the thermocouple and into the pan. The pan is cooled with a coiled, $1 / 4$ "copper tube brazed onto the bottom of the pan. The constant temperature boundary condition provided by the cooling system hastens the attainment of a steady-state fire. Including the thickness of this coil and the bottom of the pan, the overall height of the pan is $9.2 \mathrm{~cm}$. 


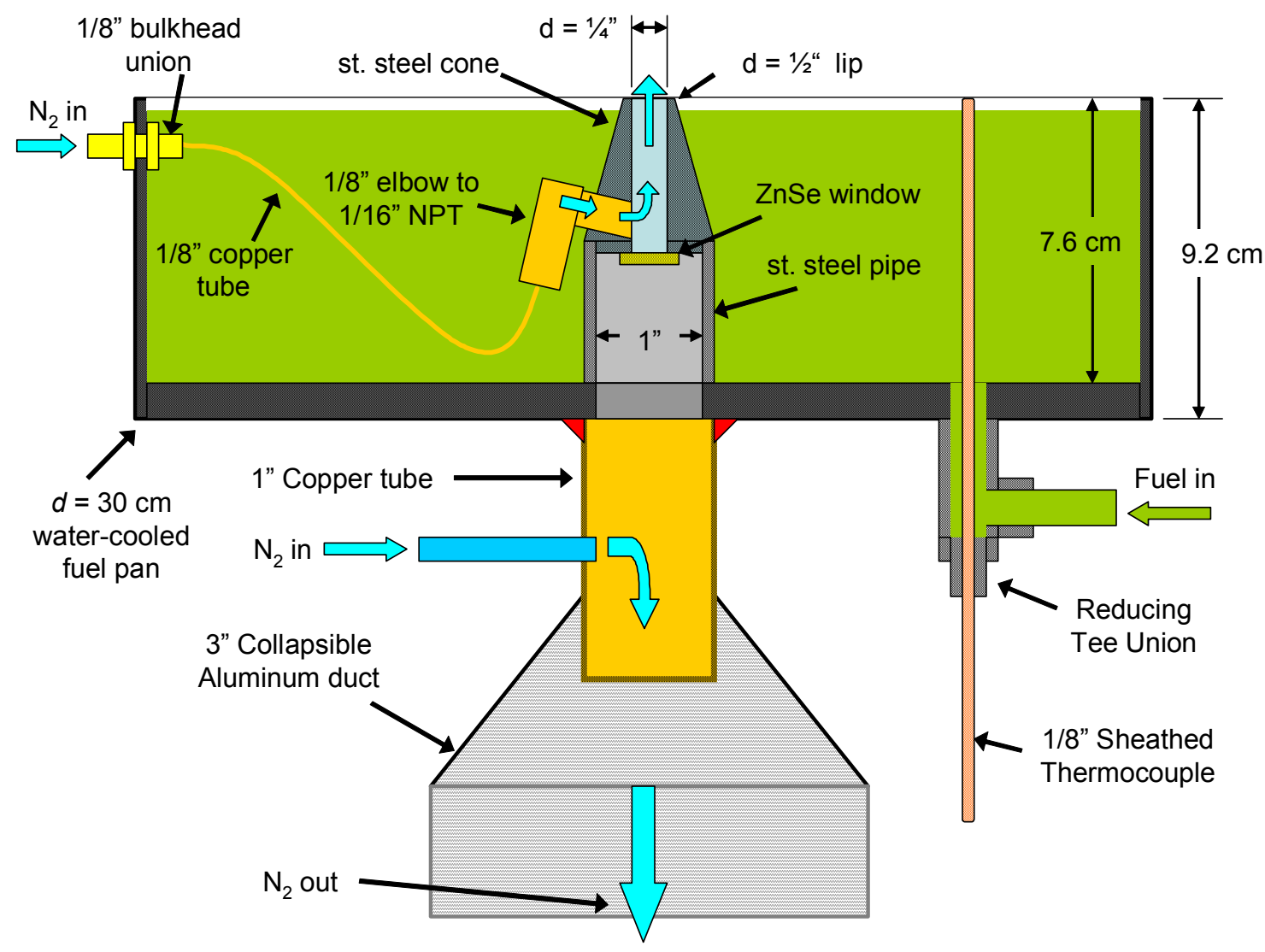

Figure 2.2: A schematic of the fuel pan with its attached features, including purge lines, thermocouple, and fuel input

\subsubsection{Optical Arrangement and Diagnostics}

The optical system of the test rig involved the most design work and assembly for this study. This system was designed to measure the infrared spectra of the pool fire, control the experimental pathlength, and maintain optical alignment.

A MIDAC Illuminator Fourier Transform Infrared (FT-IR) Spectrometer was used in the experiments. One of the most important aspects of the FT-IR design is that it comes in two separate parts: the source box and the sensor box. The model also allows the users to place the interferometer assembly (includes the beam splitter, mirrors and 
drive motor) in either box, allowing the FT-IR to operate in transmission mode or emission mode. In transmission mode, the FT-IR source is modulated by the interferometer before it passes through a sample, measuring the attenuation of the source's blackbody spectrum. Any emission inside the medium is not modulated and is not measured in this particular arrangement. This is the mode that was used in the experiments, since it provides for the simplest form of the radiative transport equation to solve for species concentrations in the fire. Alternately, the emission mode places the interferometer assembly right before the sensor. Therefore, any emission in the medium, in addition to the blackbody source, will be measured and interpreted spectrally by the FT-IR.

The MIDAC spectrometer is marketed for its rugged design for use in harsh environments. However, to insure that the optics was not affected by the fire, the FT-IR was placed outside the sheet metal fire enclosure. Two 1.5" diameter access holes were cut into the enclosure walls to allow a line of sight for the FT-IR. The source box was placed near to ground level and the beam is aimed at a mirror to direct it through the fire, while another mirror directs it back towards the sensor, which is mounted on a tripod above the source box.

The source was placed below the fire to make analysis easier. Since the beam enters the fire from the fuel surface level, the boundary condition is constant. If the beam entered from above, the boundary condition for each height measurement would change and make data analysis difficult. Data analysis is discussed further in Chapter 3.

A water-cooled, angled probe, as shown in Figure 2.3, was adapted for this study. 


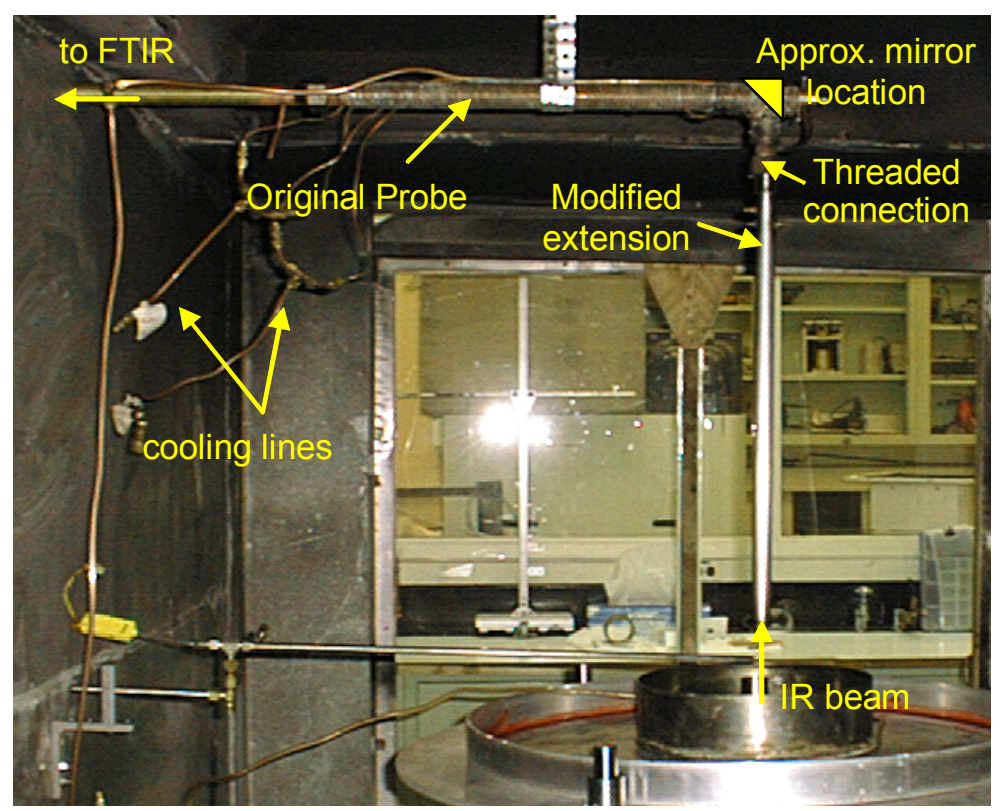

Figure 2.3: A labeled photograph of the partially-assembled angled probe and probe extension used in pool fire experiments

The probe was constructed of two 1" brass pipes brazed together at a 90-degree angle. The length of the probe with an extension piece, approximately $1 \mathrm{~m}$, was sufficient to span the distance from the FT-IR sensor outside the enclosure to the centerline axis of the fuel pan. The right-angled junction of the probe was positioned above the centerline of the fire. A 45-degree angle, first-surface gold mirror at the junction of the two pipes reflects the FT-IR beam coming from underneath towards the FT-IR sensor. The "cylindrical" mirror is placed inside the probe on the end of a brass rod, inserted through the open end of the horizontal pipe. The positioning of the mirror for alignment was adjusted manually for maximum FT-IR signal, and its position was fixed by tightening a small machine screw threaded through the brass pipe, which acts as a setscrew on the cylinder. The angled probe is cooled by many turns of $1 / 8$ " copper tubing brazed onto the exterior of the pipe; the pressure drop from the cooling line required the installation 
of a water pump. The heat protection system was augmented with a layer of Kaowool insulation and a wrapping of aluminum foil to protect the system from irradiation and from penetration by hot gases. The performance of the cooling system was monitored with a $1 / 16$ " diameter, sheathed, type $\mathrm{K}$ thermocouple inserted into the brass rod, next to the cylindrical mirror. The thermocouple was measured throughout a fire with a data acquisition system described in Section 2.3. Its value during methanol tests was usually $\mathrm{T}<30^{\circ} \mathrm{C}$, suggesting that cooling system was adequate. A $1 / 4$ " copper tube was soldered into the probe near the FT-IR sensor for $\mathrm{N}_{2}$ purging and a zinc selenide $(\mathrm{ZnSe})$ window was placed on the same end of the probe as a cap, forcing the entire $\mathrm{N}_{2}$ purge to flow out of the flame end of the probe. ZnSe was chosen for its high transparency in the infrared region and for its robustness in fire environments. The position of the probe's end above the fuel surface determines the experimental pathlength.

The original vertical extension pipe of this probe, which was attached with a nut tightened onto the two threaded ends of pipe, was deemed insufficient for the experiment. Initial trials of the cooling system for the angled probe suggested insufficient flow rates and its short vertical length would have required placing the large probe directly into the fire for many tests, possibly affecting the flame structure. A new, longer probe extension with a different cooling arrangement was designed and fabricated, and is shown in Figure 2.3. A probe extension with a length of $60 \mathrm{~cm}$ was chosen to allow the probe to stay a distance above the pool surface and the so-called "fuel-rich core" zone of the pool fire, even when the experimental pathlength is short. An inner diameter of $1 / 2$ " allowed a large area for the FT-IR beam to pass through while maintaining adequate signal strength. Providing cooling while keeping the outer diameter down to 1" was the goal of the 
design. The probe extension is a water-cooled design with flow between concentric tubes separated by ring spacers and sealed with caps welded onto the ends. Swagelok fittings were welded onto the exterior tube for the inlets and the outlet of the coolant. The flow was channeled up and down along the entire length of the tube by the strategic placement of notches in the spacer rings and by channeling the flow with thin wires. The effectiveness of the cooling system was evaluated locally by exposing the tube to a propane torch during cooling, where water condensed on the tube. Further validation of the entire tube was performed in preliminary methanol pool fires by monitoring the exit temperature of the coolant, which was only lukewarm. Overall, the cooling systems were more than capable in preventing significant thermal expansions and/or stresses in the angled probe assembly.

The angled probe assembly was cantilevered over the fire to prevent any deformations of support members, which would compromise optical alignment during fire tests. The sensor-end of the probe is fixed by U-bolts and attached to a beam installed overhead in the fire enclosure. Metal blocks were sandwiched between the beam and the probe to act as spacers to adjust the position of the probe. Adjusting the Ubolts' positions together changes the height of the probe, while relative changes of the two bolts would create an angle to the probe, which was useful for aligning the probe and compensating for cantilever beam deflection at the end of the probe.

The FT-IR beam is passed through purged tubes for a majority of its pathlength in order to minimize spectral radiation absorbance by ambient air or combustion reactants and products. The FT-IR beam enters an $\mathrm{N}_{2}$ purged 1" copper tube almost immediately after it leaves the FT-IR source box. The tube is open on the FT-IR end and the purge 
enters in the midpoint its length. The experiment is not very sensitive to the purge rate in this tube and a flow rate is chosen capable of creating enough positive pressure to overcome the intrusion of ambient gases into the optical pathlength. The FT-IR beam is aimed at a first-surface, $5 \mathrm{~cm}$ square aluminum mirror mounted at 45 -degrees onto a small aluminum plate. The plate rests on the ground perched upon three machine bolts threaded through the plate at opposing ends. These bolts serve as setscrews which can modify the tilt and height of the plate and aids in the optical alignment of the FT-IR. The mirror is enclosed inside a vertical $15 \mathrm{~cm}$ long, 3" diameter PVC pipe adhered to the aluminum plate with RTV, which also acts as a sealant. A 1" hole drilled into the side of the PVC pipe allows it to receive the FT-IR beam and the accompanying 1" copper tube. This arrangement is shown in Figure 2.4.

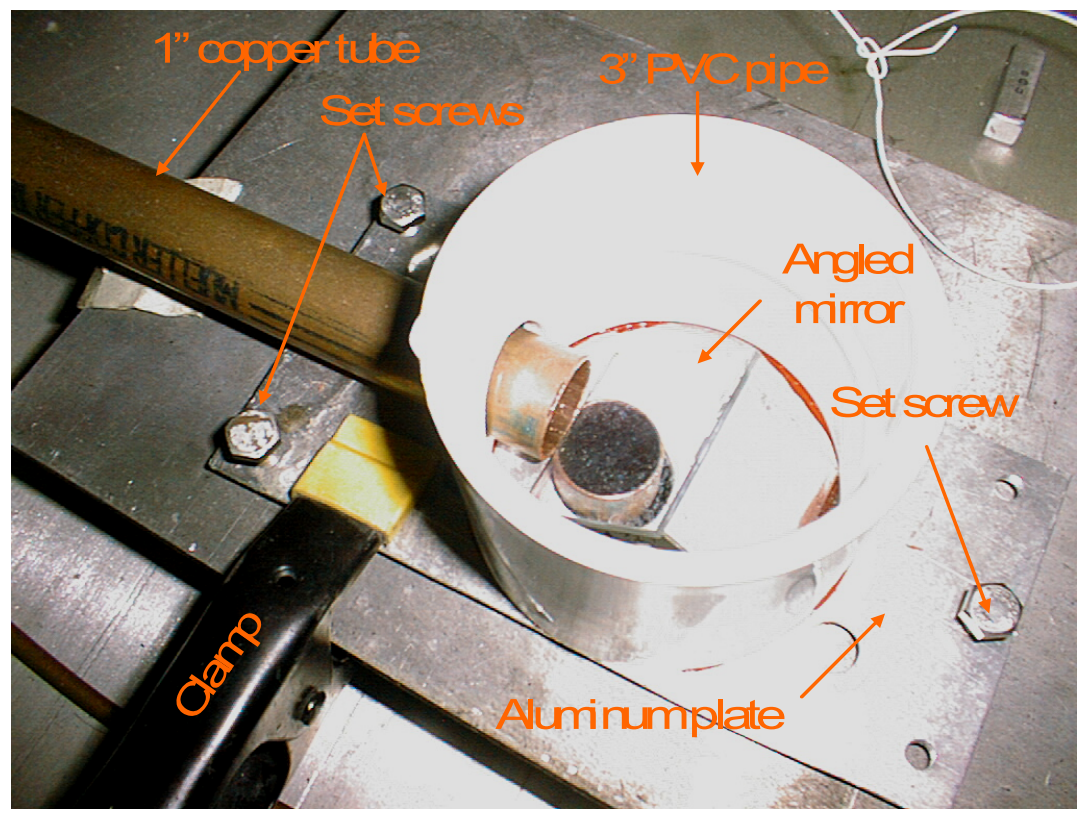

Figure 2.4: Picture of angled mirror below the fuel pan, aluminum plate and set screws used for optical alignment, and tubes used for purge containment 
The top of the PVC pipe receives a flexible, collapsible, 3" diameter aluminum duct. The collapsible duct provides a purged area for the FT-IR beam from the mirror to the fuel pan that is easily adjustable for various height measurements. The length of the aluminum duct was chosen such that at the duct's maximum extension, the fuel pan was able to be lifted adjacent to the end of the angled probe while the duct stayed connected to the mirror below. When the duct is fully collapsed, the fuel pan is $>50 \mathrm{~cm}$ below the angled probe, which was more than required clearance for the measurements taken for this experiment. In order to affix the duct to the fuel pan, an approximately $10 \mathrm{~cm}$ long piece of 1"copper tube was adhered to the bottom of the fuel pan hole using heat-resistant silicone adhesive (RTV). The RTV proved to be a tough enough material to withstand the fire environment while it provided a seal for the purge and supported the weight of tube, duct, and purge line. The aluminum duct was attached fully extending a few inches of the end of the duct, feathering the end with shears, folding it over the end of the copper pipe, and adhering and sealing the connection with foil duct tape. A 1/4" copper tube soldered into the 1" copper tube section provides a connection for the purge line.

The original purpose of this purge line was to provide $\mathrm{N}_{2}$ for the entire optical pathlength between the mirror and the fuel surface, with purge flowing in two directions: down through an aluminum duct to the mirror, and up through a 1" diameter stainless steel pipe towards the flame. One purpose of the purge was to prevent any entrainment of $\mathrm{O}_{2}$ through the center of the fire, which would create a premixed flame regime. The premixed flame was noticeable during the fire and the $\mathrm{N}_{2}$ flow was increased during setup until the premixed flame disappeared. However, the original design had very little control over how much of the $\mathrm{N}_{2}$ went in either direction, and the preliminary FT-IR 
results showed concentrations of methanol near the pool surface about one-third of what was anticipated from an FDS simulation of a $d=30 \mathrm{~cm}$ methanol pool fire run by Wakatsuki et al., ${ }^{20}$ suggesting that there was a large flow of $\mathrm{N}_{2}$ diluting this area of the fire. In a separate experiment using the purge flow rate recorded during fire tests, a hotwire anemometer was used to measure the purge velocity, which fluctuated slightly between $17-18 \mathrm{~cm} / \mathrm{sec}$. This value was used in an FDS simulation of a replica methanol pool fire as a vent of $\mathrm{N}_{2}$ entering the center of the flame. The simulation showed a small but significant area of dilution in the fuel-rich zone, yielding concentrations closely matching the preliminary results.

The first approach to limit the purge velocity entering the fire was to narrow the exit diameter central tube in the fuel pan, thereby creating more of an obstruction to flow and more pressure drop, theoretically resulting in less flow and velocity. The resulting cone is shown in Figure 2.2 and is also pictured in Figure 2.5. The cone was designed to be shrink fitted onto the end of the 1" diameter stainless steel pipe. When tests were conducted with the new design, the results were not considerably different. Another anemometer test confirmed that the velocity of the purge was still too high. More interestingly, after the purge was shut off, there was still a velocity of air exiting the cone; this was due to the suction created by the ventilation hood. The $\mathrm{N}_{2}$ that would be needed to counter this induced flow of air was too high for the experiment to succeed, and the next modification needed to block this flow entirely. 


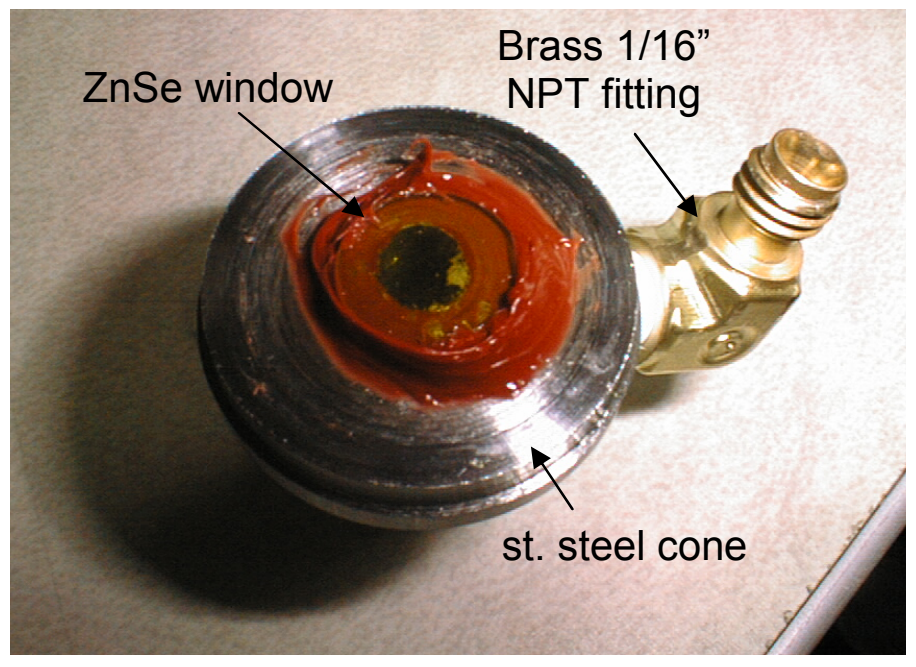

Figure 2.5: Bottom view of stainless steel cone with attached $\mathrm{ZnSe}$ window and brass Swagelok $1 / 16$ " NPT to $1 / 8$ " tube fitting

The final design included another purge $\mathrm{N}_{2}$ line going directly to the cone and separating this flow from the purge beneath with a $\mathrm{ZnSe}$ window. The window was fixed onto the cone with RTV “gasket maker" that serves as adherent and as a sealer, as shown in Figure 2.5. Enough RTV must be used to make an airtight seal but too much could cause the silicone to squeeze out and into the line of sight for the FT-IR beam. The purge enters the cone via a $1 / 16$ " NPT threaded hole through the angled wall of the cone. The placement of the hole is such that it intersects the axial hole of the cone near the bottom, next to the window. This arrangement purges the length of the cone with $\mathrm{N}_{2}$ and also prevents vaporized methanol from entering the cone and condensing on the window, which would otherwise compromise its transparency. The purge line is brought to the cone from the side of the pan and under the fuel level so that it does not interfere with the flame itself. It is attached to the cone with a Swagelok $1 / 16$ " NPT to $1 / 8$ " tube union, elbow-shaped so that it does not break the fuel surface. The $1 / 8$ " tube in the pan is connected to a purge line through the side wall of the fuel pan by means of a $1 / 8$ " 
bulkhead union, with the hole in the pan wall sealed around the union with washers and RTV.

With the new, well-contained system, the flow rate was calibrated with a DryCal DC-Lite. Positive pressure can be provided with a small amount of flow, and since little flow is needed to keep methanol from diffusing to the window, flow rates were kept at 15 $\pm 2 \mathrm{~mL} / \mathrm{min}$ during the tests, corresponding to an average flow velocity of $0.79 \pm 0.11$ $\mathrm{cm} / \mathrm{s}$ from the $1 / 4 "$ exit diameter of the cone. This flow rate was well within the desired flow and subsequent experiments resulted in methanol concentrations much more in line with expectations.

To mitigate the effects of changing boundary conditions and air entrainment patterns with variations in fuel pan height, the fuel pan was placed on a moveable mounting plate. The plate size was chosen to have a large enough area to serve as an air flow boundary. The plate is positioned onto (3) 1" diameter, steel loading-bearing rods. Linear bearings fastened onto the plate allow for the smooth vertical translation of the plate along the rods. The plate rests on collars tightened onto the rods, and moving these collars will determine the plate's position. The plate is leveled by adjusting each collar independently. The plate was manufactured with a diameter of $91 \mathrm{~cm}$ with holes appropriately placed for the fastening of (3) 1" inner diameter, flange-mount linear ball bearings, spaced $4.5 \mathrm{~cm}$ from the outer lip of the plate and $120^{\circ}$ apart. A $6^{\prime \prime}$ diameter hole was left in the middle to allow for the laser beam to pass through the plate and to allow cooling lines, fuel, and a thermocouple to access the pan.

The plate was designed to limit plate deflection from fuel and pan weight, without the overall weight becoming too heavy to lift by hand. Aluminum was chosen as the plate 
material because of its high strength-to-weight ratio. An analysis of possible plate thicknesses was completed using the deflection equation for a simply supported, pointloaded annular plate from Marks' Standard Handbook for Mechanical Engineers. ${ }^{29}$ With an expected fuel load of $20 \mathrm{lb}$, a plate thickness of 3/8" was chosen, with an estimated plate deflection due to loading and plate weight of $<0.63 \mathrm{~mm}$.

In addition to providing a platform for the fuel pan, the plate served as a secondary containment for the fuel. A $5 \mathrm{~cm}$ wide, $2.5 \mathrm{~mm}$ thick aluminum strip was bent into a $75 \mathrm{~cm}$ diameter loop fastened with rivets, and sealed onto the translating plate around the fuel pan with RTV gasket maker. This served as an axisymmetric, $5 \mathrm{~cm}$ high barrier to contain any minor fuel spills or fires resulting thereof.

\subsubsection{Fuel Supply System}

A steady fuel level is required for proper FT-IR measurements along a specified pathlength, requiring the replenishment of fuel as it burns away. A 10-liter, polypropylene carboy (Cole-Parmer, EW-62507-15) was chosen as a fuel reservoir for its good chemical resistance and effective volume to sustain a few hours of burning. This reservoir was kept on a platform above the maximum fuel level in order to allow the fuel to feed into the pan via hydrostatic pressure. The carboy came equipped with a spigot for local shutoff. A $1 / 4 "$ copper tube was fitted to the valve via a $1 / 4$ " Swagelok tube to $3 / 8$ " NPT union that was tightened into the spigot and sealed with RTV. The copper line was attached to a solenoid valve, which served as the main control valve of the system, described in more detail later in this section. 
This valve from the fuel reservoir connects to one part of a tee union, one line going to the test pan and the other going out for drainage. All three lines, including the one from the fuel reservoir, all have valves for shutoff control and to allow air to be bled from the lines, as shown in the schematic in Figure 2.6. Air is bled from the lines by loading fuel into the system and opening all of the valves until the flow of air bubbles has ceased.

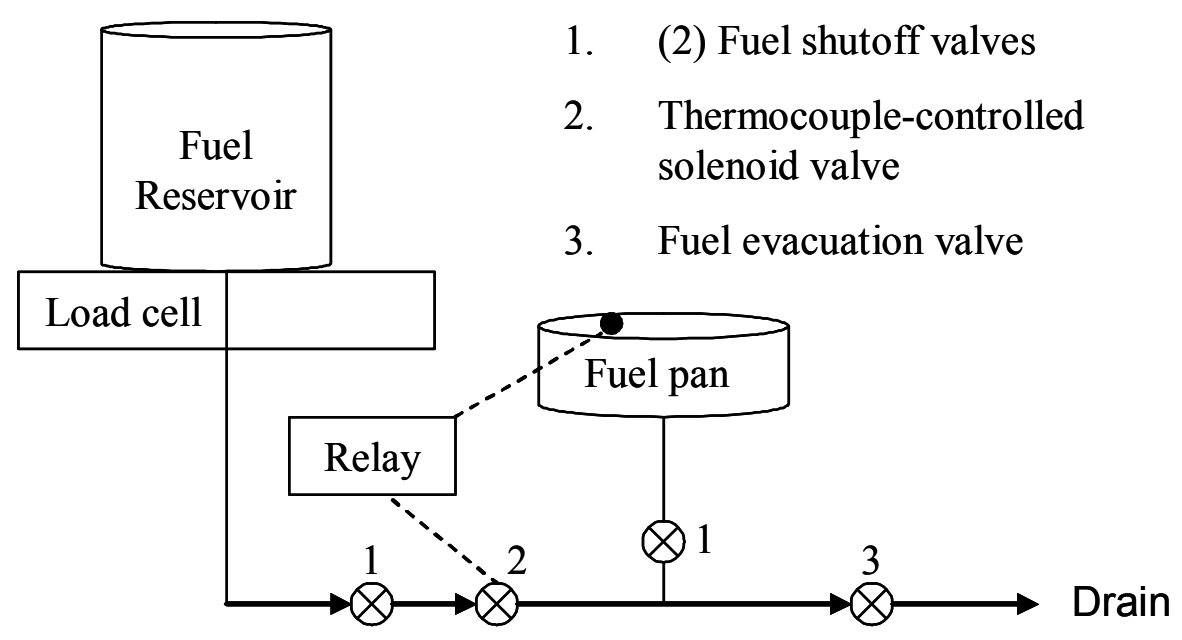

Figure 2.6: A schematic of fuel lines and valves controlling the flow of fuel during fires

Because of the requirements of flexibility during height adjustments and of chemical resistance, $1 / 4$ " PTFE tubing is used for the segment of the feed line connected directly to the fuel pan. This short tube segment is shielded from direct irradiation by the large translating plate, so it is not at risk for melting. However, this is not the case for the entire line, so the remainder of the connection is made with $1 / 4$ " copper tubing.

The solenoid valve is controlled by an Omega Process Control Relay (Model 4001KC) connected to a K-type sheathed thermocouple in the fuel pan. The 
thermocouple tip is located near the desired fuel level, as shown in Figure 2.2. As the fuel level drops during a fire, the thermocouple becomes more exposed to the flame and rises in temperature. The relay turns on the solenoid valve as the temperature rises about a specific set-point, typically around $T_{\text {vap }}$ of the fuel (for methanol: $T_{\text {vap }}=65^{\circ} \mathrm{C}$ ), and it stays open until the rising fuel level cools the thermocouple below the set-point temperature. The fuel level was maintained slightly below the pan's lip to allow a margin of error for the control system and for errors during manual pan height adjustments. Also, the height of the thermocouple was limited to less than the lip height by the snuffer used to extinguish the fire; a flat snuffer needed to be placed flush on top of the pan without interference from the thermocouple. When the relay temperature set-point is set above $T_{\text {vap }}$, the fuel level settles slightly below the tip of the thermocouple. After a burn test, the height of the fuel during the tests was measured to be $5 \pm 0.5 \mathrm{~mm}$ below the pan lip. This distance between the lip and the cone is important because it was not analyzed by the FT-IR, and the treatment of this region will be discussed in Chapters 3 and 4 .

As a pool fire burns, the liquid fuel and its surroundings heat up as a result of the energy from the reaction. As a result, less total energy is required to vaporize the fuel and the hot surroundings increase the amount of irradiation to the fuel surface, causing an increasing burn rate during the first few minutes of a fire. Once all the heat transfer reaches steady-state, the burn rate ceases to increase. This steady-state condition is the desired scenario for FT-IR measurements, and measuring the fuel burn rate is the method used to determine when a steady-state fire has been attained.

To quantify the burn rate, the fuel reservoir was placed on an electronic scale (Precisa 5000D-12000G), which outputs differential voltage varying linearly with load. 
A National Instruments SCC-68 module, PCI-6220 card data acquisition (DAQ) system was installed on a computer with a LabVIEW program to gather the scale voltage. Using standardized masses, the system was calibrated to measure mass by finding the two linear equation parameters to convert volts into grams. The 16-bit voltage readings were read at a rate of $100 \mathrm{~Hz}$ and averaged over one second in order to increase the signal-to-noise ratio of the voltage reading from the load cell. The voltage values were processed in the LabVIEW VI to convert volts to grams. In order to provide a real-time indicator of fuel burn rate, two different moving averages were calculated in real-time: an average value of the most recent ten data points, and an average of the ten data points measured starting one minute beforehand. Floating averages were used to reduce the noise in the signal, and also to smooth out intransient measurements caused by intermittent fuel flow. The difference of the two average mass measurements, divided by one minute, gives the fuel burn rate. The result was plotted in LabVIEW, allowing the experimenter to judge when a steady-state fire is reached by tracking the changes of the fuel burn rate.

\subsubsection{Gas-phase Temperature Measurements}

During some pool fire experiments, a $3 / 8$ " outer diameter, aspirated, type $\mathrm{K}$ thermocouple, constructed by NIST/BFRL, was used to measure the centerline temperature profile of the pool fire plume. The temperature profile was necessary to calculate temperature-dependent absorption coefficients of the various gaseous species for the computation model of radiative transfer. An aspirated thermocouple shields the thermocouple inside a tube and draws air through the tube with a vacuum pump. The

gases flow over the thermocouple bead, thereby measuring the temperature of the 
environment by forcing the heat transfer to be dominated by convection. This reduces the error created by radiation to and from a bare-bead thermocouple during measurements in hot environments. The aspiration velocity in the tube is recorded by dividing the volumetric flow rate, monitored with a rotameter, by the cross-sectional area of the tube. The aspirated gases were exhausted to a ventilation hood because of the dangerous amounts of poisonous $\mathrm{CO}$ gas drawn from the combustion zone.

The measurements were conducted in conjunction with FT-IR measurements and recorded by the DAQ system at a rate of $100 \mathrm{~Hz}$. Measurements were then averaged across one second to provide each data point. The thermocouple was mounted inside the enclosure just off of the fire centerline and $2.5 \mathrm{~cm}$ below the end of the angled light probe. As the pan height was adjusted, the thermocouple provided data points near the centerline profile in $5 \mathrm{~cm}$ increments, starting at the initial pan height minus the distance to the thermocouple $(5 \mathrm{~cm}-2.5 \mathrm{~cm}=2.5 \mathrm{~cm}, 7.5 \mathrm{~cm}, 12.5 \mathrm{~cm} \ldots)$.

One drawback of using an aspirated thermocouple in this experiment is that as combustible gases are vacuumed from the fire, reactions can continue inside the aspiration tube. This reaction can cause higher temperature measurements than the targeted local temperature, since the bead of the thermocouple is setback somewhat from the entrance of the tube. This is a source of error that is difficult to quantify. Results from the temperature profile measurements and their comparison to previous studies are presented in Chapter 3. 


\subsubsection{Gas-phase Species Measurements}

Gas-phase species concentration data was collected with an Agilent 3000A MicroGC Gas Chromatograph (GC) to validate the experimental procedure and to provide a comparison for the FT-IR experimental measurements. A 316 stainless steel concentric tube probe designed for annular flow of coolant was used to extract gas samples. The probe has an outer diameter of $1 / 2$ " and an inner diameter of 0.18 ", and gas samples were drawn at a rate of $1 \mathrm{~L} / \mathrm{min}$. The coolant used in the probe was dimethylphenylmethylsiloxane, CAS No. 63148-52-7, which was pre-heated to prevent the condensation of $\mathrm{H}_{2} \mathrm{O}$ and methanol in the probe during sampling. Coolant entered the probe at $100^{\circ} \mathrm{C}$ and exited at $115^{\circ} \mathrm{C}$. The remainder of the sampling line to the $\mathrm{GC}$ was also heated with electrical heating tape to prevent condensation.

Samples were drawn and analyzed at a frequency of $1 / \mathrm{min}$. after the fire had reached a steady-state and enough gas had moved through the sample line to purge the last sample. The probe was attached to a block on a threaded rod, which was elevated remotely with an electric motor. The fire was sampled along various points along its centerline without it being required to extinguish it.

\subsection{Experimental Procedure}

Proper experimental procedures were especially important in these tests because of the nature of fires. They are dangerous, difficult to measure, and sensitive to their environments. Recreating identical conditions for each measurement is difficult because of the many purge lines, cooling lines, and because of the pan movement and changing atmospheric conditions. However, several systems, such as rotameters, enclosed purge 
lines and cooling, were employed to make the test as repeatable as possible and to keep the boundary conditions similar for each test. All these systems would be useless unless a proper testing procedure was followed for each test. Complex equipment, such as the GC, was professionally calibrated, and the electric scale and the cone purge rate were calibrated by the experimenter. A working $\mathrm{CO}$ detector and fire extinguisher must be present in the room prior to any tests.

\subsubsection{Alignment}

One of the most onerous tasks for this experiment was the alignment of the FTIR. The FT-IR pathlength is approximately 5 meters long, reflects off of two mirrors, goes through the pan and two other tubes; each of these factors adds to the difficulty of alignment. Using higher quality optical mounts would have eased the process somewhat, but budget constraints did not allow for this option.

First, the angled probe mirror was aligned by sight. The exit of the probe should be visible through a properly aligned probe. If it was not visible, and if the mirror is properly mounted on its rod with the probe at a true right angle, then the mirror can be easily brought into alignment by rotating the rod until the exit becomes visible. The probe was mounted directly underneath the center of the ventilation hood to provide for an axisymmetric flow of air, though this also depends on whether the hood was centered in its enclosure, which it was not. In this experiment, it was not, and it created leaning fires under certain exhaust rates. Therefore, the exhaust rate kept low to minimize this effect. 
Next, the location of the floor directly below the probe's location was marked with using a plumb-bob. This was the location for the second angled mirror, underneath the pan. The height of the mirror was made level with the incoming FT-IR beam by placing the mirror level with the FT-IR source window by placing the mirror on several wide, stable and heavy pieces of metal or brick. A level can be used on the purged tube between the mirror and the FT-IR source to fine tune this alignment.

Once the mirrors and purge tubes are installed in their proper locations, then bottom mirror is aligned. A laser aimed from the FT-IR source towards the mirror is used to track the path of the FT-IR beam. This laser should reflect from the mirror and enter the purge tube above, and the mirror was adjusted accordingly by rotating the mirror and using the setscrews installed on the mounting plate. Once mirror alignment is complete, a clamp was placed on the aluminum plate to fix the position of the mirror to the base, such that the mirror does not move from random bumps or perturbations. The clamp, however, bends the aluminum mounting plate, so fine tuning of the alignment was required after the clamp's installation.

The FT-IR sensor was mounted onto a large tripod capable of extending an adjustable platform up to $2 \mathrm{~m}$ above its base. The platform has three adjustable degrees of freedom: one translating (vertical) and two rotational (yaw and tilt). The FT-IR source was placed on a flat scissor jack to elevate the box to the proper height. The FTIR was operated in "Align" mode, which uses a quick scan rate to produce an interferogram and the signal voltage, to check the alignment of the system. The signal strength was noted, and was improved incrementally by first adjusting the alignment of 
FT-IR source box, then the FT-IR sensor box, and then using minor adjustments in the mirrors in the system to increase the signal strength.

Last, the fuel pan was placed on top of the translating plate. Ideally, the FT-IR beam should go through without attenuation, but sometimes it will be blocked if the pan is off center or if the plate is not level. Keeping the pan centered and making sure that the plate is level after any height adjustment is one important key to keeping the signal strength high and consistent throughout the experiment.

\subsubsection{Test Procedures}

The FT-IR exhibits changing frequency response throughout the tests. It is not completely avoidable, and procedure to account for the changing response will be discussed in Chapter 3, yet it is preventable to a certain degree by setting up the FT-IR and allowing it to run for at least 30 minutes before any data points are taken. Liquid nitrogen $\left(\mathrm{LN}_{2}\right)$ is used to cool the MCT detector of the FT-IR, and it is added to the sensor early, and it should be replenished during testing. The alignment of the system is noted by checking the signal voltage, and the signal voltage is allowed to reach a plateau before the testing commences, which usually takes about 15 minutes.

The water cooling lines are attached to cold water taps and are turned on full. The water pump is turned on to its pre-adjusted speed. Flow should be running at maximum and be free of any blockages. Blockages can be noticed by small changes in the flow, and proper cooling was also tested by applying a propane torch to cooled sections, such as the angled probe extension. 
The $\mathrm{N}_{2}$ purge originates from a single gas cylinder, which is regulated to $30 \mathrm{psi}$ during the experiment. The main line branches out to the four purge lines with their own valves and rotameters. The rotameter heights are adjusted to match the experimental flow rate requirements for each test, which were determined by evaluating the purges' effectiveness during preliminary pool fire tests. It is important to activate the purge before the test because it will protect various optical parts from heat, condensation of methanol and water, and soot deposition.

The pan height is adjusted to the starting height, usually $5 \mathrm{~cm}$. The levelness of the pan-mounting plate is checked, and the fuel pan position is adjusted until maximum FT-IR signal intensity is reached. The aspirated thermocouple and GC probes, if used, are mounted in place just off of the FT-IR path and their positions relative to the probe and pan are measured and recorded. The probes' sampling pumps are turned on and adjusted to the proper flow rates.

An FT-IR background measurement must be taken before any fuel enters the pan or the enclosure. This background with no fire or fuel vapors in the experimental pathlength is the basis for comparison for the first sample height of $5 \mathrm{~cm}$. The room doors are closed and all cooling and purge flows are on to mimic the experimental conditions. The FT-IR is then activated to take a background measurement between 650 and $4500 \mathrm{~cm}^{-1}$ at $0.5 \mathrm{~cm}^{-1}$ resolution, averaging the measurement over 128 scans.

Before adding fuel to the pan, it is wise to plug or tape over the cone opening so that fuel does not spill onto and damage the $\mathrm{ZnSe}$ window underneath it. The purge to the cone must be shut off before covering the hole. After fuel is added to fill the pan about halfway, all valves in the fuel feed system are opened to purge the lines of any air, 
collecting the runoff in a can. Once the lines are purged, the valves are turned off again and the remainder of the fuel pan is filled. The cone is unplugged and the purge is turned back on immediately.

Before the fire is lit, all safety measures should be ready, including having the snuffer and a $\mathrm{CO}_{2}$ fire extinguisher on standby and having observers in the room. Once all preparations are ready, the data collection systems are turned on, including the FT-IR and LabVIEW programs. The methanol pool is lit at arms length with a stick lighter, providing extra distance from the fire.

Once the fire is lit, the fuel feed system is activated by opening the valves feeding the pan and by adjusting the relay set-point. As fuel level rises, the temperature reading on the pan thermocouple drops, and the solenoid valve stops the flow once the temperature reaches its set point. The fuel system is monitored to insure that this process occurs without spillover in the pan.

Elapsed time should be recorded in either lab notes or in the data collection itself to synchronize the various data being collected for each test. As the fuel burn rate is monitored, a judgment is made when a steady-state fire has been reached, which usually takes 10 to 15 minutes. This time is in agreement with the experimental observations by Hamins et al., ${ }^{4}$ who used a similar cooled-pan arrangement. The time to steady-state burn is noted for future reference during data analysis.

When adequate data has been obtained from one pan height measurement, the fire is extinguished and the fuel pan height is adjusted. Prior to extinguishing the fire, all measurements are stopped and the fuel flow is shut off with the main safety valves. In order to allow for sloshing during the pan adjustments, the fuel is allowed to burn for a 
couple of minutes to lower the fuel level. The primary extinguisher is a snuffer made from a $35 \mathrm{~cm} \times 35 \mathrm{~cm} \times 0.3 \mathrm{~cm}$ steel plate, large enough to cover the pan with $2.5 \mathrm{~cm}$ clearance on all sides, attached to a solid beam with fittings with gasket washers to prevent any openings in the plate. A thick and heavy piece of steel was required to prevent any warping in the plate while the snuffer was exposed to the fire, which occurred in designs using thinner pieces of aluminum. Any warping or openings in the plate can provide enough oxygen to keep the fire burning. Before extinguishing the fire, a second person must be ready with a $\mathrm{CO}_{2}$ or $\mathrm{N}_{2}$ extinguisher to put out any flames the snuffer leaves exposed.

After the fire is extinguished, the snuffer is removed, the cone purge is shut off, and the cone is plugged once again before the plate is moved to the new height. During height relocations, the fuel pan is covered with a sheet of heavy duty aluminum foil to provide protection from fuel vapors and reignition; the exhaust rate is increased to clear any vapors from the enclosure. The process is repeated for all subsequent measurements.

After the last fire has been extinguished, the cooling lines may be shut off and the FT-IR and DAQ may be powered down. The pan is most safely drained through the drainage valve in the fuel feed system, shown in Figure 2.6. Once the pan is drained, the purge lines and the ventilation hood may be shut off. Results from these experiments are presented in the following chapter. 


\section{CHAPTER 3: Results of Methanol Pool Fire Measurements}

This chapter presents the results from the experiment described in Chapter 2, which includes infrared spectra, temperature, fuel burn rate, and gas concentration measurements from a $d=30 \mathrm{~cm}$ methanol pool fire. This chapter describes some trends revealed by the results, and discusses some basic conclusions that can be inferred from the data regarding the experimental setup and the flame structure. It also sets up some concepts required to ascertain gas species concentrations from the infrared spectra, which is calculated by a post-processor described in Chapter 4 .

The fundamental data for this experimental study were the intensity spectra measured by a MIDAC Illuminator FT-IR spectrometer. These spectra are important because they can provide a measure for assessing the species inside the fuel-rich core of the pool fire. This is accomplished by comparing a "background" spectrum, taken in the absence of a flame or fuel in pan, and a "sample" spectrum, which is taken during a fire. An example of the two spectra from a pool fire is shown below in Figure 3.1. The sample spectrum shows spectral absorbance of various compounds in the fire. The strength of these absorbance bands are used to calculate the concentration of each compound locally within the fire. In this chapter, the measurements used to perform this calculation and to confirm the veracity of the experimental setup are presented. 


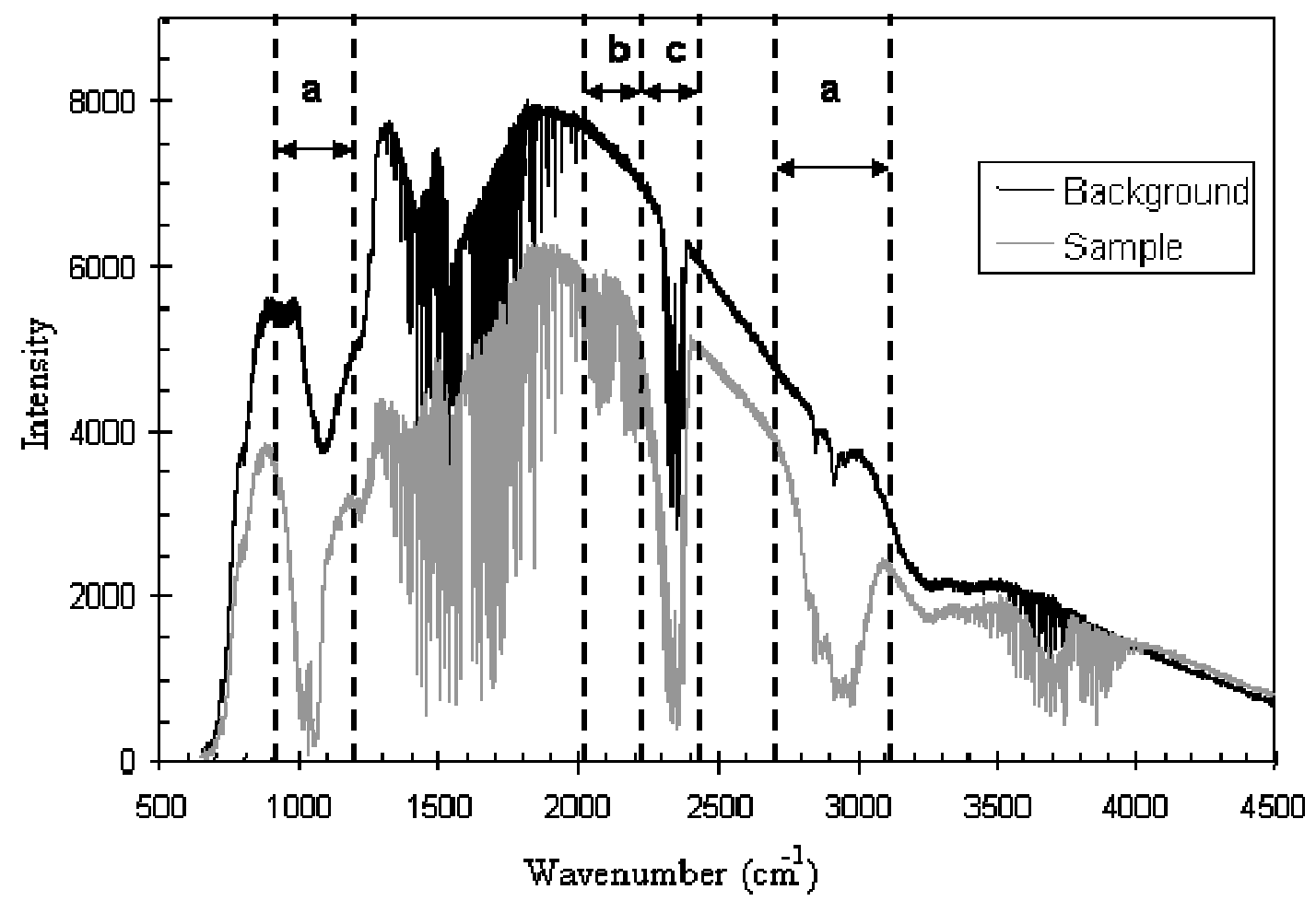

Figure 3.1: Reference (background) and sample spectra from a methanol pool fire showing characteristic spectral absorption regions of a) methanol, b) $\mathrm{CO}$ and c) $\mathrm{CO}_{2}$

\subsection{FT-IR Spectra}

Measurements inside a $d=30 \mathrm{~cm}$ methanol pool fire were taken with an FT-IR spectrometer collecting spectra between the wavenumbers of $650 \mathrm{~cm}^{-1}$ and $4500 \mathrm{~cm}^{-1}$ at a resolution of $0.5 \mathrm{~cm}^{-1}$, averaged over 64 scans. The spectra were recorded in units of relative intensity. The FT-IR was arranged in "transmission mode," as described in Section 2.1.1, such that any radiative emission from the flame was not recorded by the spectrometer. This permits the use of simpler equations of radiation transport for a computational model to calculate species concentrations from spectral data. The spectra 
recorded over progressive heights were compared to each other to calculate radiative absorbance across the fuel-rich region of the pool fire. By measuring at successive heights and comparing total pathlength absorption at the successive heights, the absorption associated with each additional pathlength segment could be evaluated.

The lip height of the pan was $0.5 \mathrm{~cm}$ during the tests. Since the cone protrudes above the fuel surface an equal distance, the FT-IR tests do not capture the absorption of compounds at the first $0.5 \mathrm{~cm}$ inside the flame, i.e. the FT-IR data may be treated as starting at an initial height of $z=0.5 \mathrm{~cm}$. Implications of this data on the analysis of species concentrations will be discussed in Chapter 4 .

\subsubsection{Intensity spectra}

Infrared spectra were recorded over the duration of a test, but only spectra recorded after the flames had reached a steady-state mean structure and burning rate were used in the analysis. Examples of recorded spectra (averaged over 64 scans) from steadystate flames are shown in Figure 3.2. The time interval between measurements at different heights, $z$, was about 30 minutes for sequential tests, and could be longer. This was the time involved in allowing the fire to reach steady-state, extinguishing it, letting the area cool, and adjusting the plate height. However, such a long time interval caused some discrepancy between measurements due to slow transient changes in FT-IR response. These changes resulted in different "background" response as a function of measurement height and required a correction, described in Section 3.1.4. 


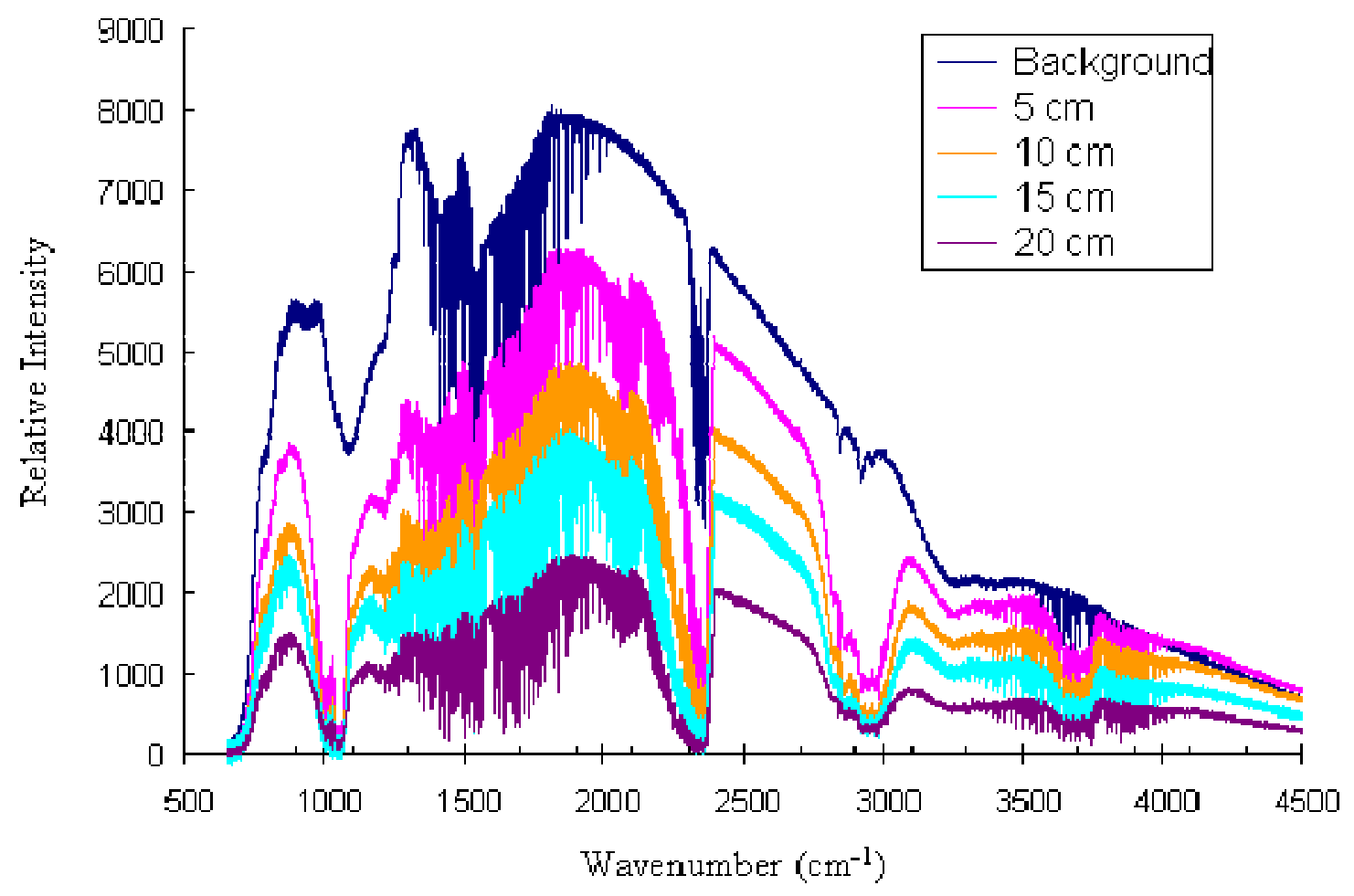

Figure 3.2: A plot of transmitted blackbody intensity spectra measured through various centerline heights $(z)$ of a $d=30 \mathrm{~cm}$ methanol pool fire

Figure 3.2 illustrates several significant trends in the spectra. First, the background spectrum shows an overall shape indicative of a blackbody source with some absorption occurring in bands centered at 1595,3657 , and $3756 \mathrm{~cm}^{-1}$ due to the presence of $\mathrm{H}_{2} \mathrm{O}$ vapor and at $2349 \mathrm{~cm}^{-1}$ due to the presence $\mathrm{CO}_{2}$ in the pathlength. ${ }^{11} \mathrm{~N}_{2}$ purging greatly reduces the presence of these gases through the pathlength but does not entirely eliminate them. The $\mathrm{CO}_{2}$ bending band about $667 \mathrm{~cm}^{-1}$ causes some absorption, but the weakness of the signal in this frequency region makes its contribution less evident. Other regions in the background spectrum showing some absorbance, particularly regions centered at 1100 and $3000 \mathrm{~cm}^{-1}$, suggests the presence of hydrocarbons in the optics of the FT-IR, since there was no fuel present at the time of the measurement. These bands 
do not invalidate the measurements, but their presence in the background must be accounted for when transmission and absorbance spectra of a sample are calculated relative to the background.

The sample intensity spectra at successive heights in the fire show several trends. First, there is a graybody-like attenuation of the signal intensity by the fire that increases with height. This is clearly illustrated in Figure 3.2, which shows a general drop in intensity with increasing height. Since there are no soot particles and associated particle scattering in the methanol flame, this attenuation is may be due to the amplification of the misalignment of the angled probe with increasing pathlength. Another potential cause of the attenuation may be the temperature and concentration gradients associated with eddies in the flame, which creates a change in the refractive index that could potentially refract the IR beam away from the probe opening. This cause may be likely, due to the higher attenuation at lower frequencies. The correction method to account for this graybody-like attenuation is discussed in Section 3.1.4.

Figure 3.1 shows the absorbance bands associated with combustion reactants and products. In Figure 3.2, the absorption bands of methanol $\left(\mathrm{CH}_{3} \mathrm{OH}\right)$, especially due to the C-O stretching peak centered about $1033 \mathrm{~cm}^{-1}$ and the C-H stretching peaks about 2844 and $2981 \mathrm{~cm}^{-1}$, are clearly visible. ${ }^{20}$ The CO absorbance peak centered about $2143 \mathrm{~cm}^{-1}$ is also clearly visible in all sample measurements. The $\mathrm{CO}_{2}$ asymmetric stretching peak centered at $2349 \mathrm{~cm}^{-1}$ exhibits increasing absorbance as a function of height due to the completion of combustion. This peak also clearly shows broadening, especially toward lower wavenumbers, at larger heights because of the higher temperatures in these regions. This effect is clearer at lower wavenumbers for $\mathrm{CO}_{2}$ absorption because a "band head" 
forms on the high wavenumber end of the band at $\omega=2410 \mathrm{~cm}^{-1}$ such that absorption does not broaden beyond it. ${ }^{23}$ All of the trends become clearer once intensity spectra are converted into transmittance and absorbance.

\subsubsection{Transmission spectra}

Spectral transmissivity $\left(\tau_{v}\right)$ is the ratio of sample spectral intensity to reference spectral intensity: it is the fraction of energy transmitted by a sample. For our case, the sample spectral intensity $I_{V}$ is the spectrum measured through the fire at height $z$. The closest approximation to a reference intensity in the pool fire experiments is the background intensity $\left(I_{v, B G}\right)$ measured at height zero before fuel is in the pan. Therefore, $\tau_{v}$ measured at height $z$ is calculated by:

$$
\tau_{v}(z)=\frac{I_{v}(z)}{I_{v, B G}(0)}
$$

Plotting $\tau_{v}$ makes it easier to identify absorbance bands of combustion species and to analyze FT-IR performance by comparing measured $\tau_{\nu}$ against the anticipated "baseline" value $(\tau=1)$. The reference and sample data shown in Figure 3.2 are used in Eq. (3.1) to produce the transmission spectra plotted in Figure 3.3. 


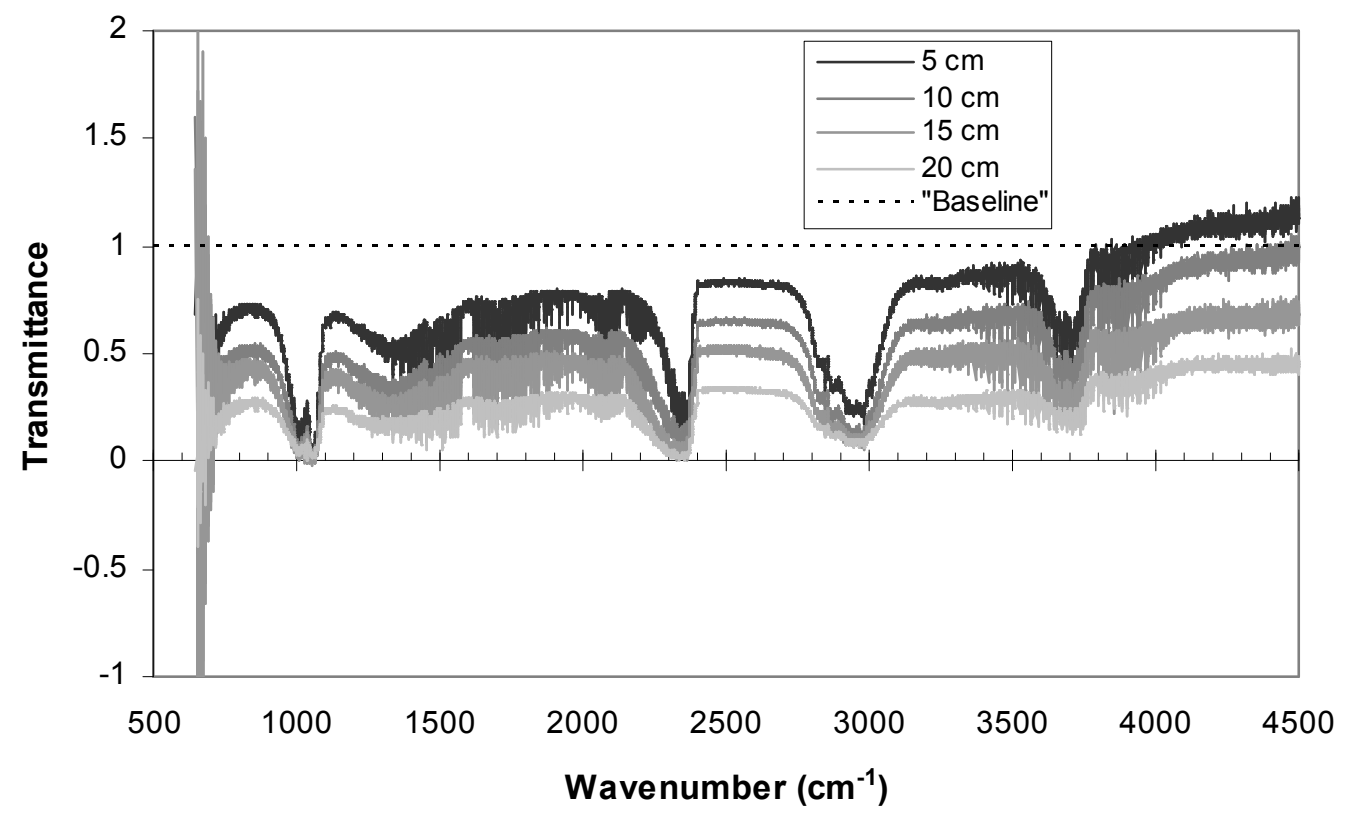

Figure 3.3: Plots of transmission spectra at various heights inside a $30 \mathrm{~cm}$ methanol pool fire

Figure 3.3 indicates several trends important for analysis. First, the bending band for $\mathrm{CO}_{2}$ centered about $667 \mathrm{~cm}^{-1}$ is very noisy due to the weak signal at low wavenumbers. Since the noise is on the same order of magnitude as the signal, this makes the band essentially useless for analysis. Most other bands show good signal strength for potential analysis. Also, the time-dependent response behavior of the FT-IR becomes clearer when plotted with $\tau_{v}$ : signal strength improves at high frequency with elapsed time. This is inferred because higher heights are measured later in the tests, and are measured well after $I_{v, B G}(0)$ is recorded. For $z=5 \mathrm{~cm}$, Figure 3.3 shows that $\tau_{v}>1$ for the region $\omega \geq 4000 \mathrm{~cm}^{-1}$, even though the overall trend of $\tau_{v}$ decreases with increasing $z$. This suggests a change in the instrument response that is independent of $z$. This time-dependent instrument response was confirmed by background measurements at 
the beginning and at the end of testing. Correction for instrument response and gray attenuation as a function of $z$ is discussed in Section 3.1.4. The least noisy regions of the spectra are between $800 \mathrm{~cm}^{-1}$ and $4000 \mathrm{~cm}^{-1}$. Also, because the signal weakens with increasing $z$, the signal to noise ratio decreases with $z$ as well.

\subsubsection{Absorbance spectra}

Transmission spectra are converted to absorbance spectra by application of eq. (1.23). As shown by eqs. (1.25) and (1.26), absorbance units provide a direct relation to species concentrations. The calculated absorbance units are linearly related to $P_{k} z$, and relative local species concentrations along the flame centerline may be compared with the graphs plotted in Figure 3.4.

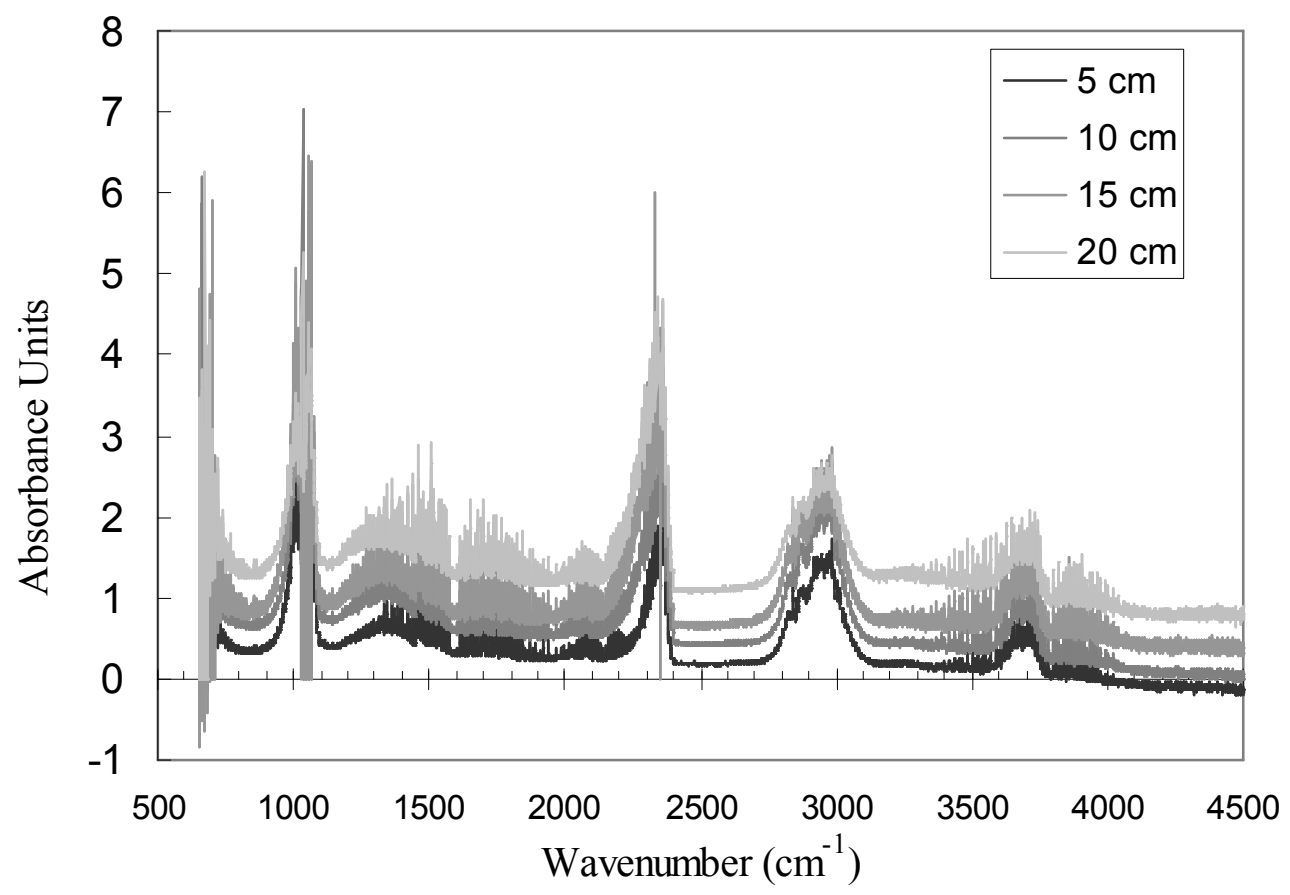

Figure 3.4: Plots of absorbance spectra for a $d=30 \mathrm{~cm}$ methanol pool fire at various heights 
This figure shows the effect of poor signal strength in certain regions. The signal is extremely noisy in the low wavenumber region which masks the $\mathrm{CO}_{2}$ bending band centered about $667 \mathrm{~cm}^{-1}$. This is a combined affect of the low signal strength in the spectrometer and the strong absorption of this band. In certain lines, extinction can be seen in the C-O stretching region centered about $1033 \mathrm{~cm}^{-1}$. "Extinction" occurs when the signal and transmittance of a line is measured to be less than or equal to zero. The absorbance, as the negative natural log of the transmittance, is not defined for this case. The plots substitute zero for this data in the plots, and the result can be especially seen in the measurements for the absorbance through $z=15 \mathrm{~cm}$ of the fire. This suggests that the optical alignment or the spectrometer performance was less than adequate. However, this consequence only makes itself apparent as the fire develops and absorption strengthens in the methanol bands, and is therefore not possible to predict this behavior before commencing a test. Since it is difficult to predict, line extinction shows up in most data sets. In order to avoid problems during data processing for data points where absorbance is undefined, the average absorbance recorded at the two neighboring wavenumbers is substituted.

\subsubsection{Correction of time-dependent instrument responses}

The FT-IR spectrometer exhibited different response profiles over the full, 8-hour tests due to a number of factors, such as cooling of the infrared sensor, loss of liquid nitrogen for sensor cooling, and mirror drift. It was difficult to compare data points taken from different heights because of the time and height dependent response profiles. To 
properly compare the background to the sample, the response of the FT-IR had to be corrected computationally. A potential method to correct for this time-dependent shift would be to take readings of the identical "background" measurement at various times throughout the day, when the FT-IR would be put through similar conditions to the testing conditions. A frequency-dependent response corresponding to each measurement as a function of $z$ is defined here.

$$
R F_{v}(z)=\frac{I_{v, B G}(0)}{I_{v, B G}(z)}
$$

where $R F_{v}$ is the spectral response function. By applying $R F_{v}$ to Eq. (3.1), the response function can adjust the measured $\tau_{v}$ to create a "corrected" transmittance, which is the ratio of the sample spectrum to a theoretical background spectrum measured under the same conditions:

$$
\tau_{v, \text { corrected }}(z)=\tau_{v}(z) \cdot R F_{v}(z)=\frac{I_{v}(z)}{I_{v, B G}(0)} \cdot \frac{I_{v, B G}(0)}{I_{v, B G}(z)}=\frac{I_{v}(z)}{I_{v, B G}(z)}
$$

The final fraction in Eq. (3.3) provides the transmissivity of interest, while $I_{v}(z)$ and $I_{v, B G}(0)$ are the only measurements available via the experimental method. Using $R F_{v}(z)$ allows the transmissivity of each test to be corrected for instrument and gray-absorption effects.

Two methods were explored to calculate $R F_{v}(z)$. The first method was to directly measure instrument response as a function of time by measuring a day-long series of background intensity spectra. The usefulness of this method is limited because it depends on identical conditions and instrument response for every FT-IR measurement being performed. Also, the FT-IR would not capture any effects related to the flame. 
Therefore, a more prudent approach is to use a second method: creating a "baseline absorbance" model of the background by fitting known non-absorbing regions of the absorbance spectra to a "baseline" curve. These spectral regions are illustrated where the transmittance (Figure 3.3) and absorbance spectra (Figure 3.4) have no spectral characteristics and are principally flat.

The correction of absorbance spectra with $R F_{v}(z)$ is shown by applying the natural logarithm to terms from Eq. (3.3):

$$
\begin{gathered}
-\ln \tau_{v, \text { corrected }}(z)=-\ln \left(\frac{I_{v}(z)}{I_{v, B G}(0)} \cdot R F_{v}(z)\right) \\
A_{v, \text { corrected }}(z)=A_{v}(z)-\ln \left(R F_{v}(z)\right)
\end{gathered}
$$

By fitting the non-absorbing regions of absorbance spectra to a curve, the correction can be calculated by subtracting the fitted curve for $\ln \left(R F_{v}(z)\right)$ from the measured absorbance.

A function was programmed into MATLAB to automate this correction process for absorbance spectra. First, the absorbance data was calculated from a background spectrum and a sample spectrum and plotted into a graph. The MATLAB function 'ginput', a graphical user interface where a user can click on a graph to return the $\mathrm{x}$ (wavenumber) and $\mathrm{y}$ (absorbance) values of these points, was used to return the $(\mathrm{x}, \mathrm{y})$ values of the non-absorbing spectral regions where the absorbance, $y$, is presumed to be zero. In addition to the user-defined points on the graph, extra data points were created by linearly interpolating for 100 data points between each of the user-defined points, $\mathrm{x}$ and y. This was done in order to give different frequency regions of the absorbance spectrum comparable weights when evaluated by a least-squares fit, regardless of the 
number or location of data points chosen by the user. The data points were fitted to an $n^{\text {th }}$ degree polynomial function using the MATLAB function 'polyfit'; a high-order polynomial is necessary to capture the logarithmic shape of the baseline. The resulting polynomial function is evaluated with the MATLAB function 'polyval,' which creates an estimate of the function $\ln \left(R F_{v}(z)\right)$. This is then subtracted from the measured absorbance, thereby completing the response function correction, as shown in Figure 3.5b.

Since they are relatively clean burning, methanol pool fires have several regions of spectral transparency. The major absorbing species present in this case are $\mathrm{CH}_{3} \mathrm{OH}, \mathrm{H}_{2} \mathrm{O}$, $\mathrm{CO}_{2}$, and $\mathrm{CO}$. Selecting frequency regions of the measured $A_{v}(z)$ that are not within any absorbance bands of these species allows the polynomial fit to find the "response function" for that particular measurement. The points chosen with 'ginput' in the study of methanol pool fires were focused on the wavenumbers just below the C-O stretching region centered at $1033 \mathrm{~cm}^{-1}$, just above the $2000-2400 \mathrm{~cm}^{-1} \mathrm{CO}$ and $\mathrm{CO}_{2}$ stretching regions, and in the $4000-4500 \mathrm{~cm}^{-1}$ region without considerable absorption. An example of points chosen on an absorbance spectrum using 'ginput' is shown in Figure 3.5a. Choosing the appropriate number and location of points to apply a response correction to a measured absorbance spectrum requires experiential knowledge of the equipment response as a function of frequency.

Applying this process to all of the measurements allows the direct comparison of the different heights for the first time. By plotting the bands specific to a particular molecule, the plots will provide an accurate representation of the concentration of that molecule throughout the various positions along the centerline of the fire. 

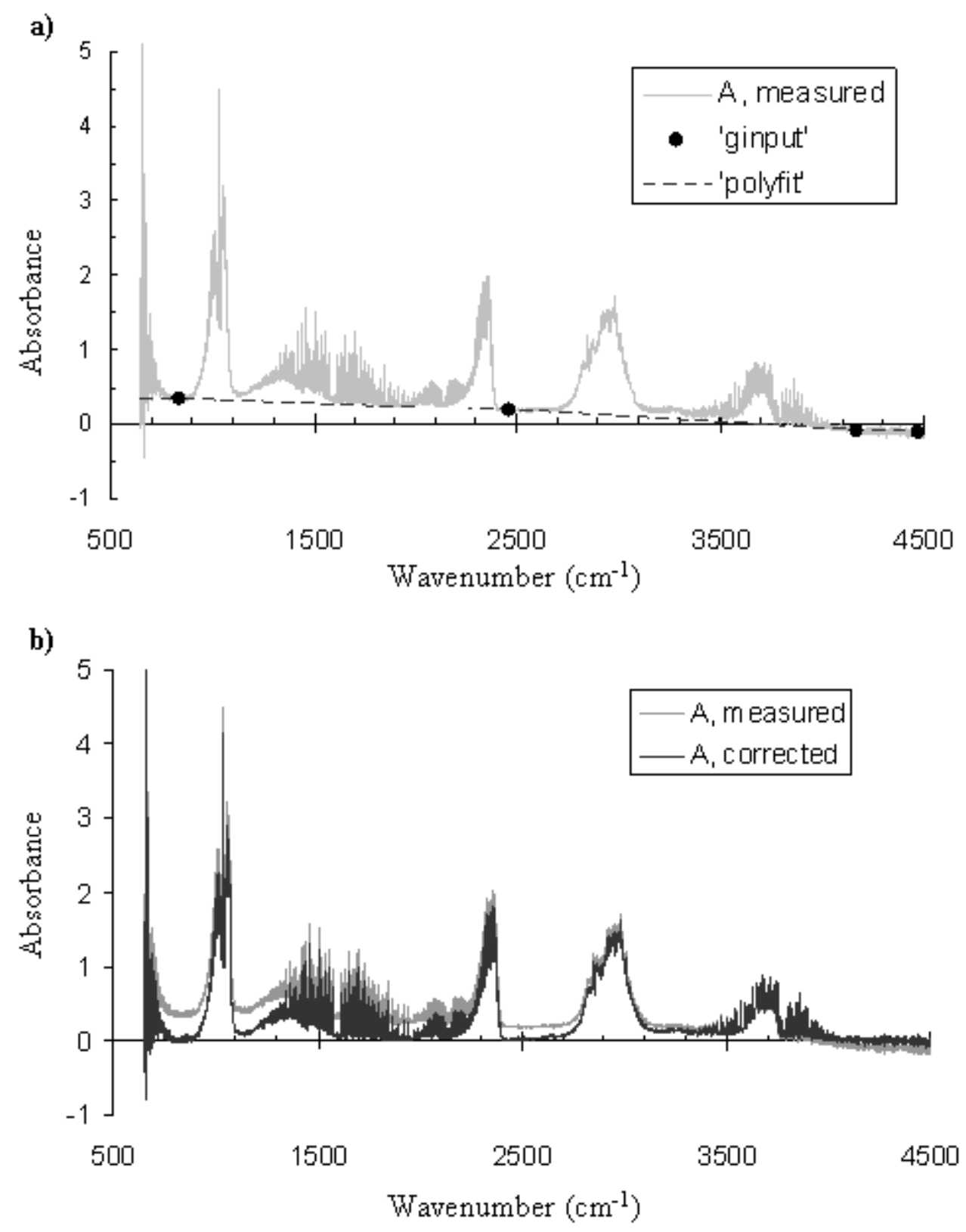

Figure 3.5: Absorbance plots illustrating correction method with a) user inputted points ('ginput') and the resulting MATLAB polynomial fit ('polyfit'), and b) the corrected spectrum 
Figures 3.6 through 3.8 show the corrected absorbance profiles of $\mathrm{CH}_{3} \mathrm{OH}$, $\mathrm{CO} / \mathrm{CO}_{2}$, and $\mathrm{H}_{2} \mathrm{O}$, respectively, through various measurement heights, $z$. Figure 3.6 shows $\mathrm{CH}_{3} \mathrm{OH}$, which is a compound that is expected to have strong absorbance near the fuel surface, and then quickly decay away. The results show that this trend generally holds true. However, for both absorbance bands shown in the graph, there is an anomaly that shows weaker absorbance at $z=20 \mathrm{~cm}$ than at lower heights. This might be explained by changes in the fuel burn rates that were observed for different measurement heights, which is discussed in Section 3.2. Figure 3.6b shows the C-H stretching band of methanol, which overlaps the absorption band of $\mathrm{CH}_{4}$. Because the information provided in the C-O stretching (Figure 3.6a) is of comparable quality, that band will be preferred for the purposes of data analysis. Some extinction was observed in Figure 3.4 for the $z=$ $15 \mathrm{~cm}$ measurement in the $\mathrm{C}-\mathrm{O}$ stretching band of $\mathrm{CH}_{3} \mathrm{OH}$, and the effect on the central peak is shown in Figure 3.6a. While the general trend shows stronger absorbance for $z=$ $15 \mathrm{~cm}$ when compared to $\mathrm{z}=5$ and $10 \mathrm{~cm}$, the central peak is not as strong because it was lost to extinction. Regardless, errors in concentration calculations will be limited when the entire band is considered.

Figure 3.7 shows trends that are expected with $\mathrm{CO}$ and $\mathrm{CO}_{2}$. The absorbance of CO does not increase dramatically past the first couple of data points, and it shows some trends consistent with $\mathrm{CH}_{3} \mathrm{OH}$ absorbance at various height measurements. The absorbance of $\mathrm{CO}_{2}$ continuously strengthens and broadens at higher measurements. This is because $\mathrm{CO}_{2}$ continues to be present at these heights and the temperature reaches a maximum in this zone (described in Section 3.3). 
Figure 3.8 shows $\mathrm{H}_{2} \mathrm{O}$ bending absorption, and it shows overlaps with $\mathrm{C}-\mathrm{H}$ bending from $\mathrm{CH}_{3} \mathrm{OH}$ between 1200 and $1600 \mathrm{~cm}^{-1}$. The trend shows the strongest absorbance occurs at $\mathrm{z}=0.05 \mathrm{~m}$, and weakens somewhat higher in the flame. 

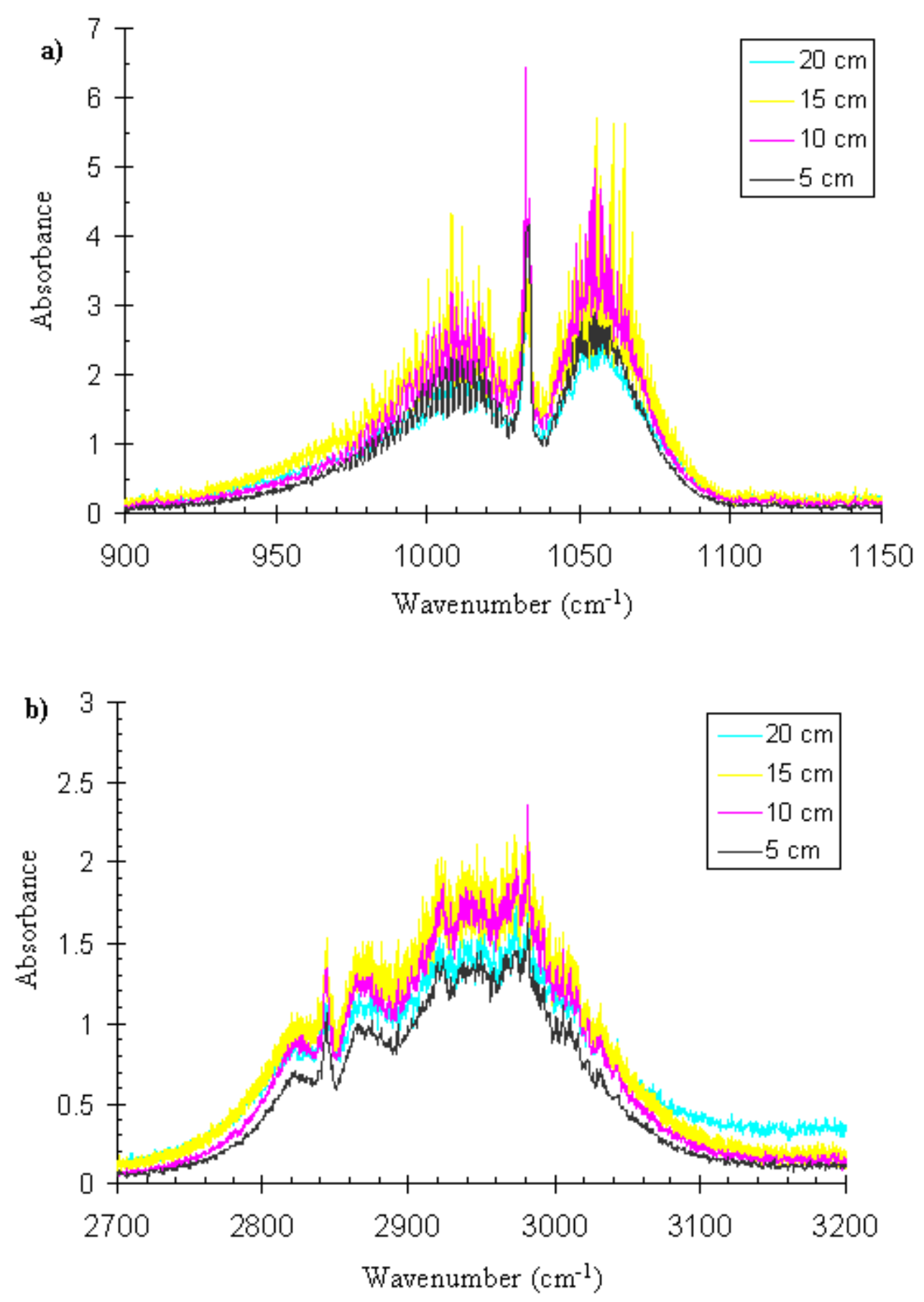

Figure 3.6: Corrected absorbance plots for at measured heights $(z)$ for the methanol vibration-rotation absorption bands due to a) $\mathrm{C}-\mathrm{O}$ stretching, and b) $\mathrm{C}-\mathrm{H}$ stretching (with methane) 


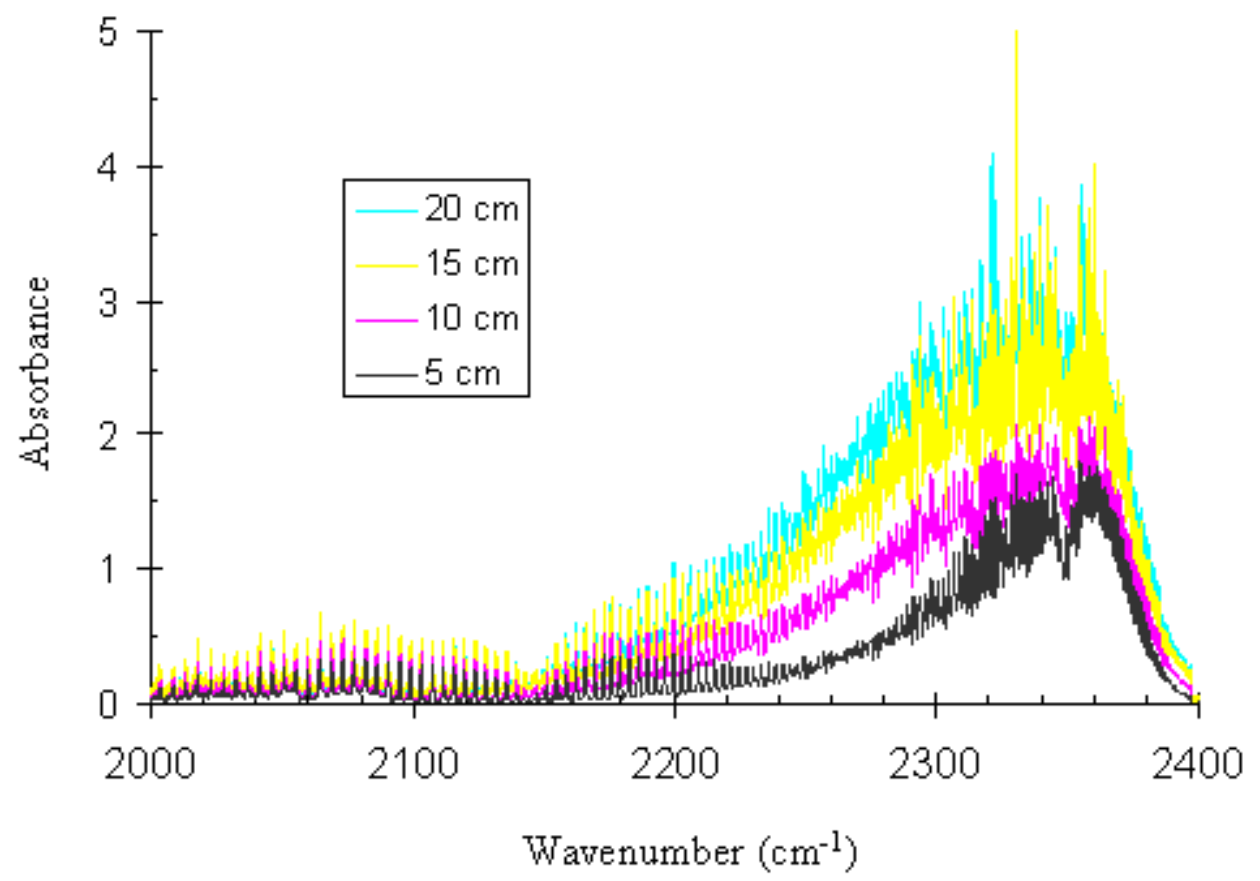

Figure 3.7: Corrected absorbance spectra for $\mathrm{CO}$ stretching and $\mathrm{CO}_{2}$ asymmetric stretching vibration-rotation absorption bands

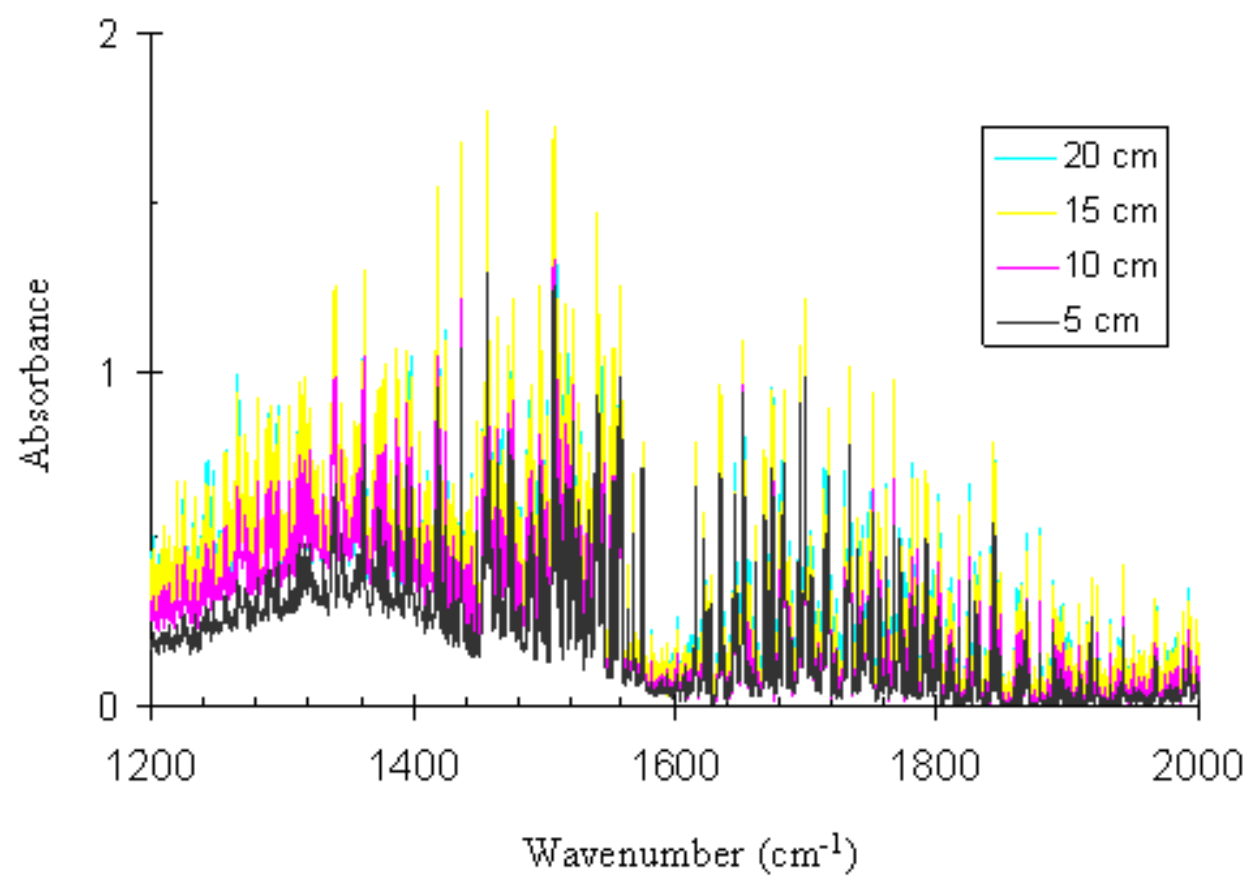

Figure 3.8: Corrected absorbance spectra for the $\mathrm{H}_{2} \mathrm{O}$ bending vibration-rotation absorption band 


\subsection{Mass Burn Rate Measurements}

A load cell was used to measure the weight of the fuel reservoir feeding the pool fire. By plotting this data, the slope of the resulting data curve could be used to estimate the fuel burn rate. This data was available to the experimenter in real-time through LabVIEW data acquisition for judgment of when the fires had reached steady-state conditions, and it was also saved for future analysis.

The fuel burn rate, $\dot{m}$, was calculated from the slope of a best-fit linear line from the electronic scale measurements as a function of time using the MATLAB function 'polyfit'. The measurements during the end of each test were used because this was the time during which the fire burned steadily. The measurements of $\dot{m}$ from two separate test days are plotted in Figure 3.9. The similar trend in both measurements is probably not a coincidence. This suggests that either the change of air entrainment patterns due to pan movement or the presence of the water-cooled probe may have affected the fire. Accumulating quantities of $\mathrm{H}_{2} \mathrm{O}$ in the fuel pan, via diffusion from vapor, may also have been responsible the observed downward trend in burn rate, though attempts to measure $\mathrm{H}_{2} \mathrm{O}$ concentrations in fuel samples after each burn using a $\mathrm{GC}$ were inconclusive. However, the measurements do not vary more than $5 \%$ from the average steady-state value of $\dot{m}=0.90 \mathrm{~g} / \mathrm{sec}$, which is in good agreement with measurements made by Hamins et al. $(\dot{m}=0.93 \mathrm{~g} / \mathrm{sec}) .{ }^{4}$ 


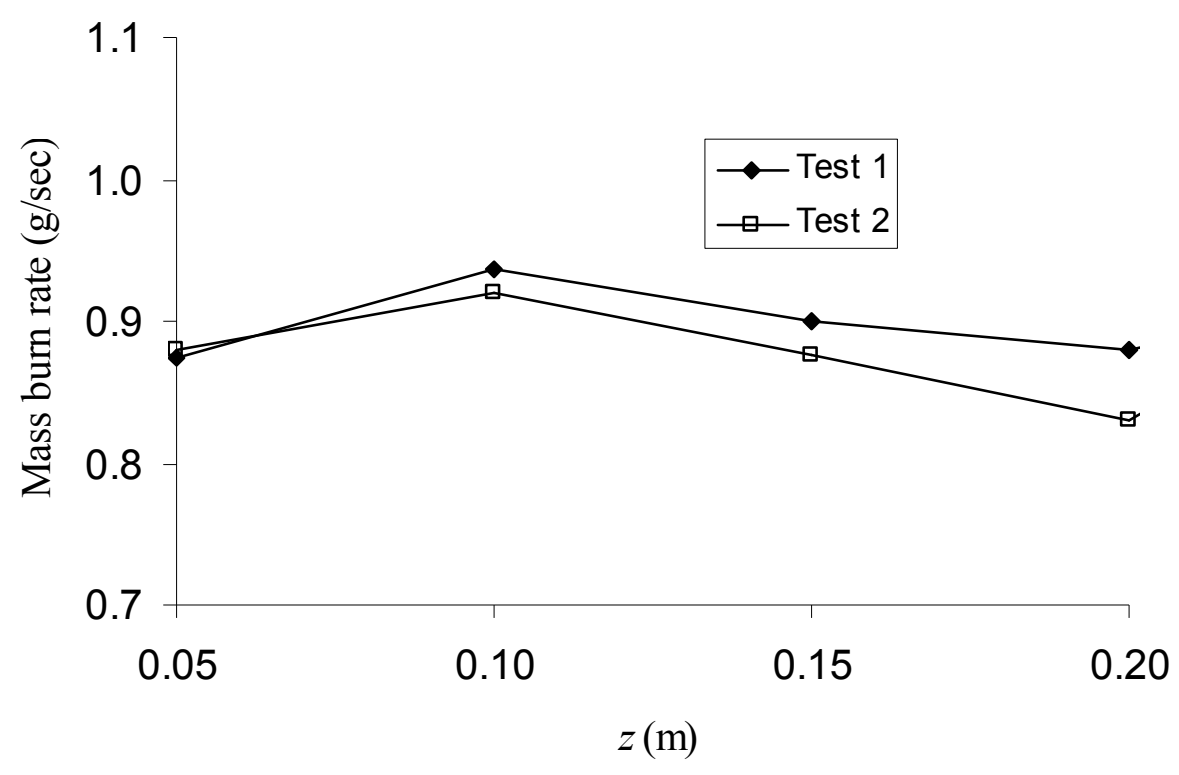

Figure 3.9: Plot of fuel burn rate $(\dot{m})$ measurements versus probe height $(z)$ in $d=30$ $\mathrm{cm}$ methanol pool fire

The mass burn rate might explain some trends noticed in the absorbance spectra of methanol, shown in Figure 3.6. Low $\dot{m}$ corresponding with the measurements at $z=$ $20 \mathrm{~cm}$ also corresponds to the lower than expected absorbance strength of methanol. High $\dot{m}$ corresponding with measurements at $z=10 \mathrm{~cm}$ shows a maximum in methanol absorption. Since $\dot{m}$ is a direct measurement of the vaporization rate of methanol, it is logical that these two results would parallel each other. Ramifications of this result on the radiative transfer model will be explored in Chapter 4.

\subsection{Temperature Measurements}

An aspirated, K-type thermocouple was used to gather temperature measurements. Temperature data was scanned at $100 \mathrm{~Hz}$ and averaged over 3 seconds 
before plotting (300 scans per data point). The time-averaged mean temperature, $T$, was calculated from a representative plot near the end of each fire test, as plotted in Figure 3.10. Error bars in $z$ represent the spatial location error of the thermocouple position and the fact that aspiration draws in samples from a surrounding neighborhood. Error bars for $T$ represent one standard deviation in the fluctuating measurements. The data shows good agreement with previous experimental studies of $d=30 \mathrm{~cm}$ methanol pool fires, ${ }^{21}$ but shows considerable deviation from FDS models at grid resolutions of $1 \mathrm{~cm}$ and $2 \mathrm{~cm}$ using the methane absorption coefficients.$^{20}$

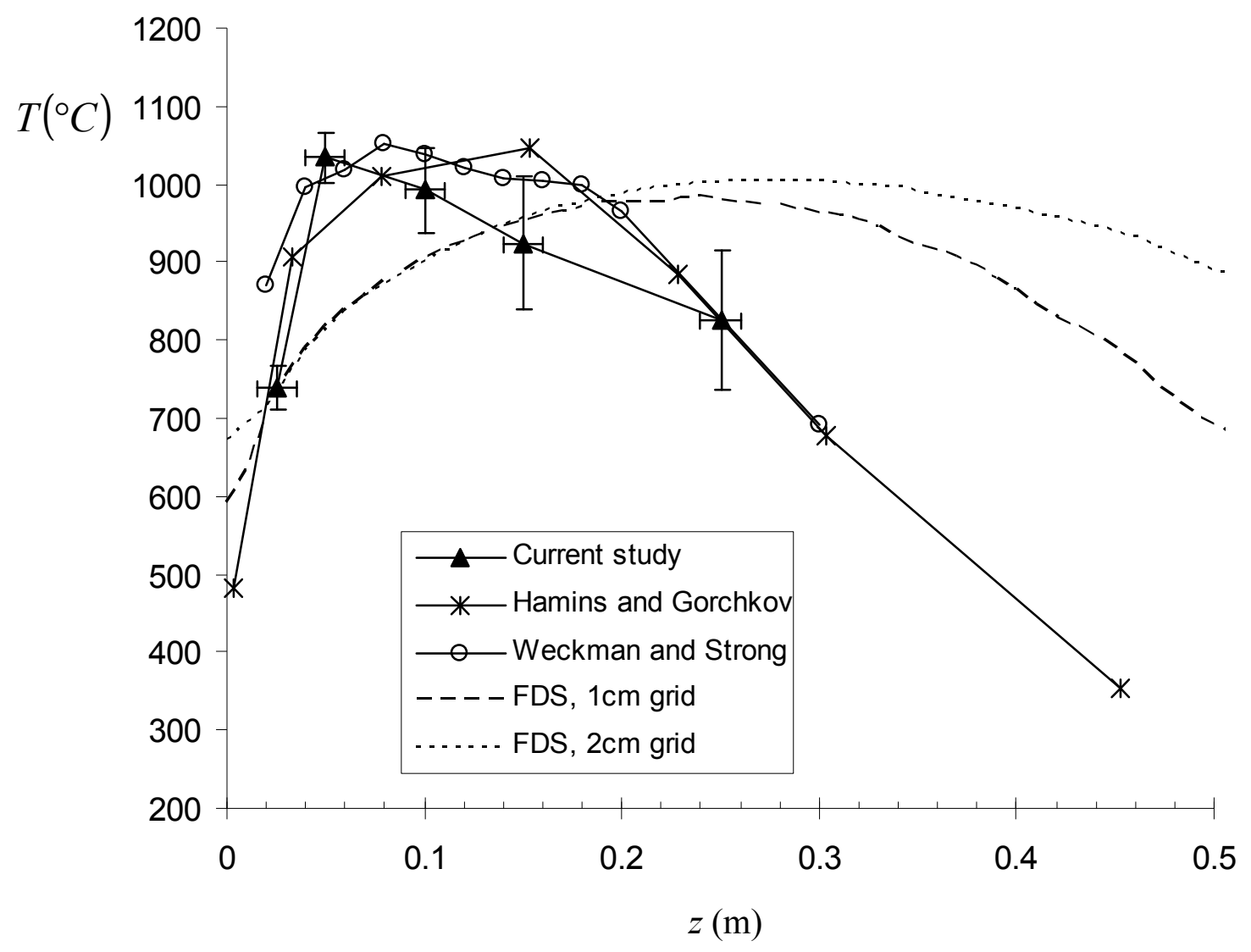

Figure 3.10: Comparison of centerline mean temperature measurements $(T)$ versus height above fuel surface $(z)$ 
Temperature profiles in the fuel-rich core are very important when calculating species concentrations due to the temperature-dependence of absorption coefficients

$\left(\kappa_{v, k}{ }^{\prime}\right.$. The absorption characteristics of all compounds change as a function of temperature, with absorption being strongest at low temperatures. Therefore, it is crucial to accurately characterize temperatures in the high $d T / d z$, fuel-rich zone of the pool fire when studying species concentrations.

The fluctuations of temperature also create issues when attempting to predict concentrations from intensity measurements due to the non-linearity of eq. (1.18) and the temperature-dependence of $\kappa_{\nu}$. The FT-IR spectrometer records the average intensity over 64 scans, corresponding to an approximate duration of one minute. This scan time easily captures the average of turbulent effects in the fire, which has a characteristic frequency equal to the pulsation frequency of a $d=30 \mathrm{~cm}$ methanol pool fire, recorded by Hamins et al. as $2.8 \pm 0.3 \mathrm{~Hz} .{ }^{4}$ The non-linearity of eq. (1.18) becomes an issue when using average intensity to calculate average concentrations. In this study, the turbulence correlation terms are assumed to be zero. This is a fair approximation when studying the fuel-rich core, as it experiences much smaller fluctuations than regions further above it, as illustrated by the temperature uncertainty bars in Figure 3.10.

\subsection{Gas Chromatography Measurements}

Gas Chromatography (GC) measurements were taken inside the subject $d=30$ $\mathrm{cm}$ methanol pool fire with the sampling probe described in Chapter 2.1.4. Figure 3.11 and Figure 3.12 show the volume fractions $X$ for major species measured from $z=1.5 \mathrm{~cm}$ 
to $55 \mathrm{~cm}$. In addition to the species shown, $\mathrm{CH}_{4}, \mathrm{C}_{2} \mathrm{H}_{4}$ and $\mathrm{C}_{2} \mathrm{H}_{2}$ were also measured in trace amounts. The sum of the volumetric fractions often exceeded $100 \%$, especially for low values of $z$, suggesting an error in the experiment or the instrumentation. The values for $\mathrm{H}_{2} \mathrm{O}$ particularly seem high, suggesting issues relating to condensation or calibration of the GC. A normalization of the data was done by dividing by a summation of volume fractions for each height:

$$
X_{n o r m, k}=\frac{X_{k}}{\sum_{k} X_{k}}
$$

The resulting values of $X_{\text {norm }}$ are shown in Figure 3.13 and Figure 3.14.

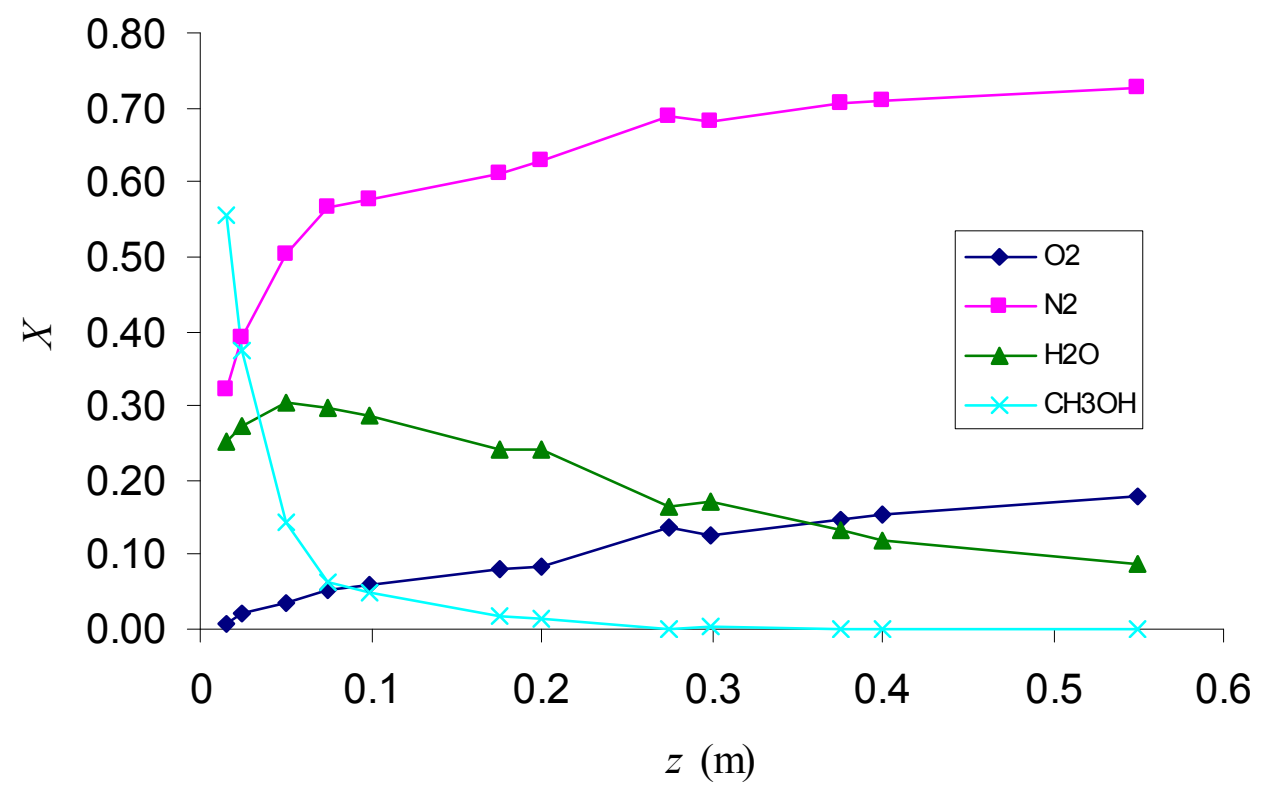

Figure 3.11: Molar fractions $X$ of major gas species along the centerline of a $d=30 \mathrm{~cm}$ methanol pool fire 


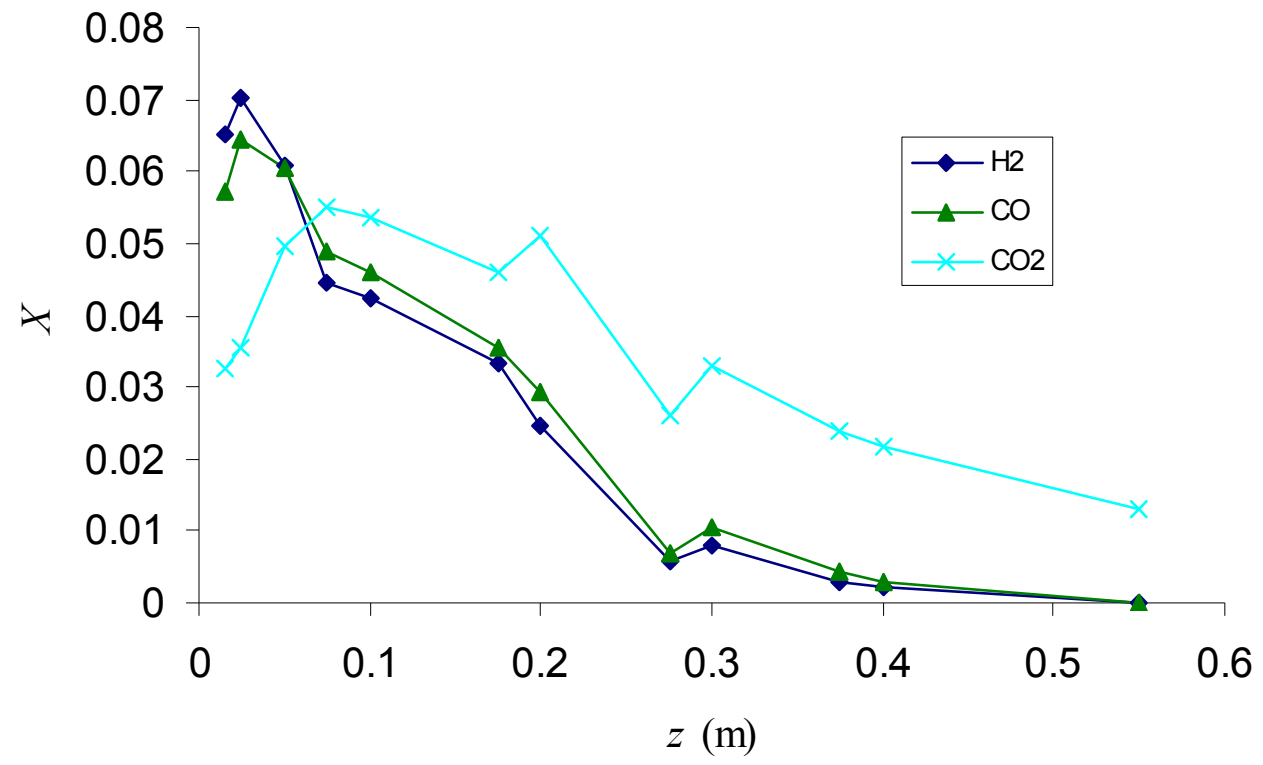

Figure 3.12: Molar fractions $X$ of minor gas species along the centerline of a $d=30 \mathrm{~cm}$ methanol pool fire

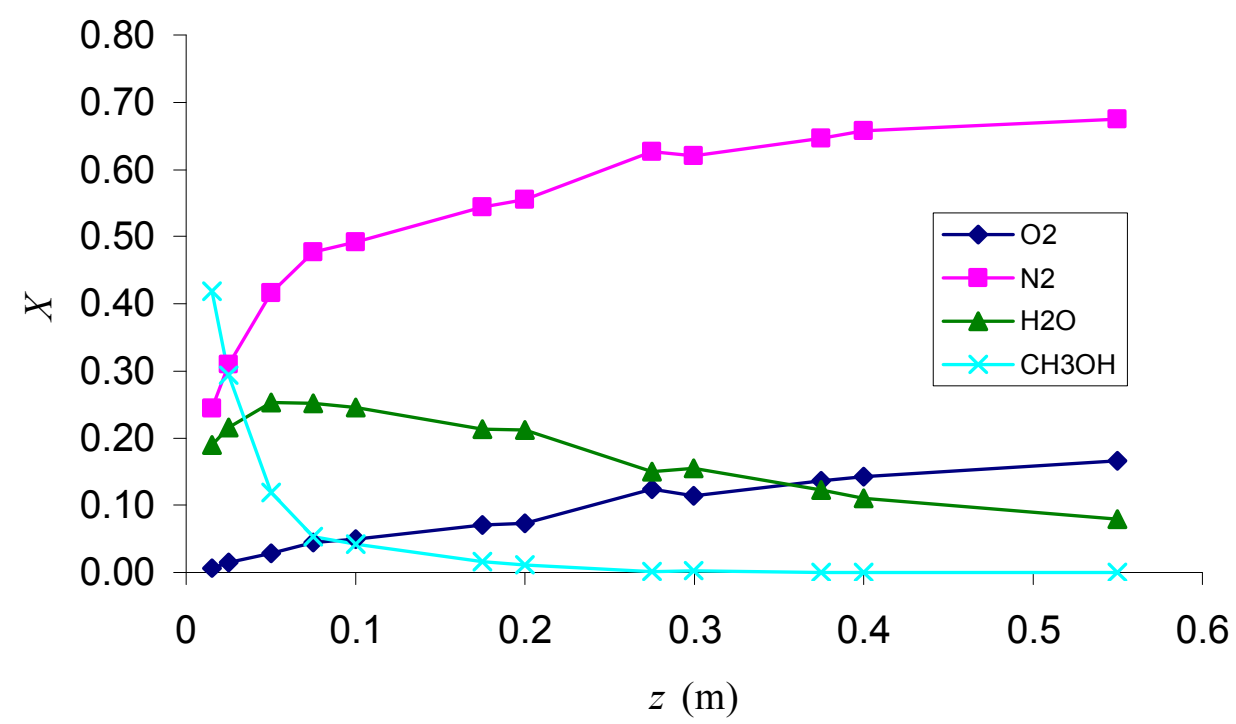

Figure 3.13: Normalized molar fractions, $X_{\text {norm }}$, of major gas species along the centerline of a $d=30 \mathrm{~cm}$ methanol pool fire, according to eq. (3.6) 


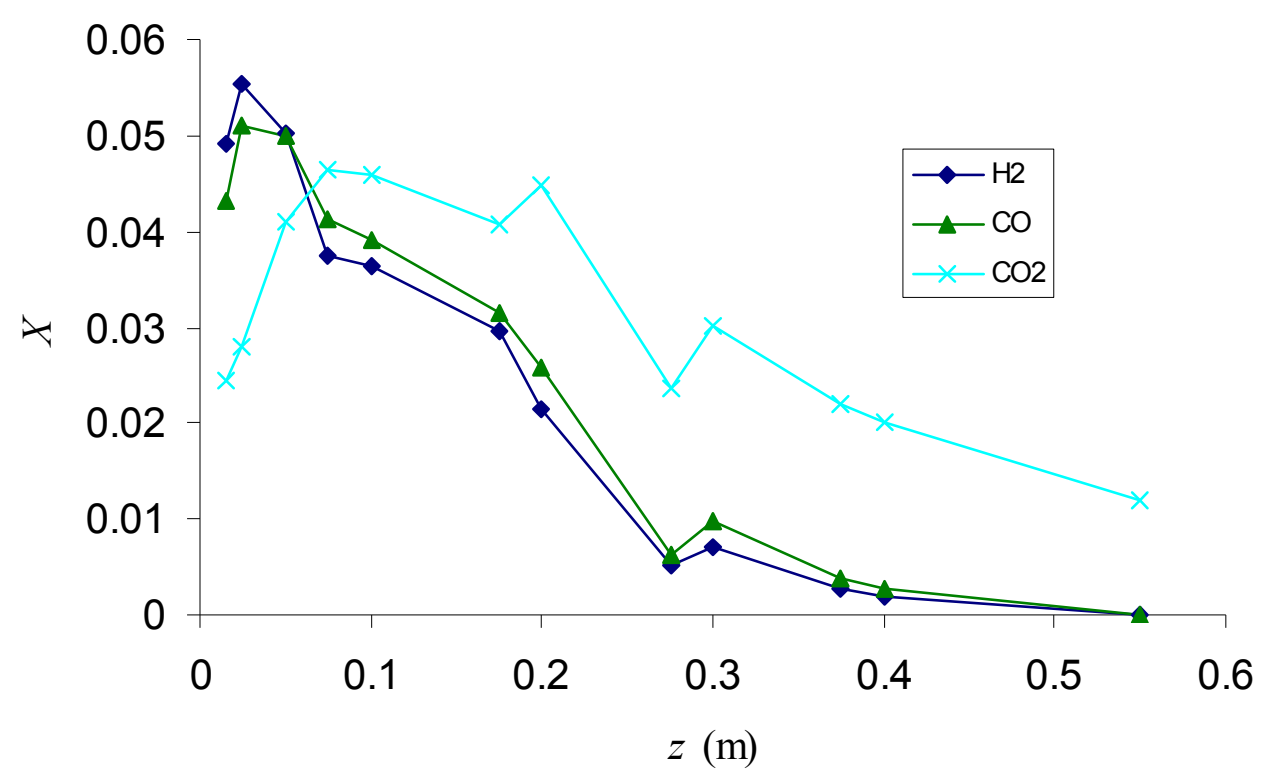

Figure 3.14: Normalized molar fractions, $X_{\text {norm }}$, of minor gas species along the centerline of a $d=30 \mathrm{~cm}$ methanol pool fire, according to eq. (3.6)

\subsection{Photographs}

Photographs of the test were collected to study several aspects of the fire, such as fire shape, fuel height inside the pan, and the stagnation point of the counter flow between the angled-probe nitrogen purge and the fire flow itself. Figure 3.15 shows pool fire tests in progress at two different measurement heights. Photographs showed where the counter-flowing flame and $\mathrm{N}_{2}$ probe purge reached a stagnation point, as shown in Figure 3.16. Occasionally, as shown in the photograph and inset of the figure, the turbulent flame boundary will flow across the counter-flow, creating a signature convexshaped flame boundary at the tip of the probe. The distance from the stagnation point to the probe is assumed to be less than the distance of the flame boundary to the probe. The 
distance of the stagnation point from the probe end was calculated to be $<0.6 \mathrm{~cm}$ by comparison to the probe's outer diameter, $d=2.54 \mathrm{~cm}$.

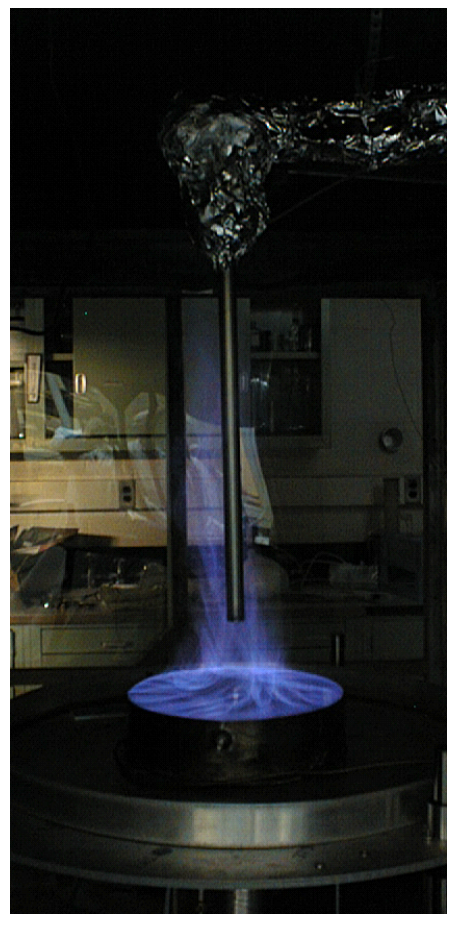

$z=10 \mathrm{~cm}$

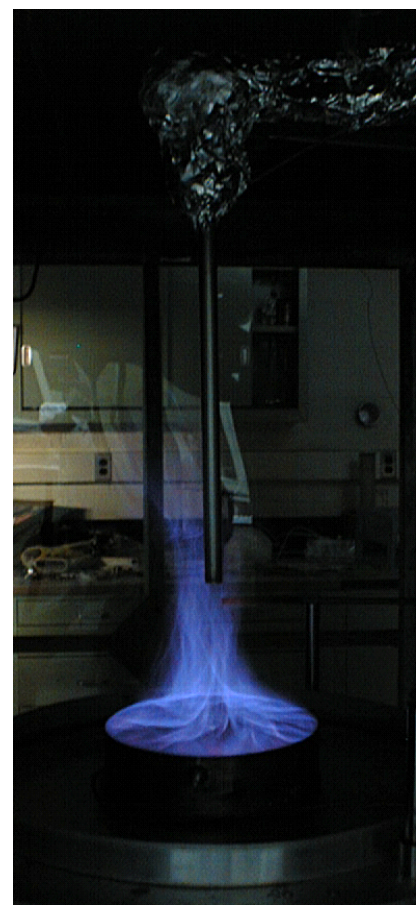

$z=20 \mathrm{~cm}$

Figure 3.15: Photographs (2) showing the methanol pool fire test rig at two different probe locations, $z$ 


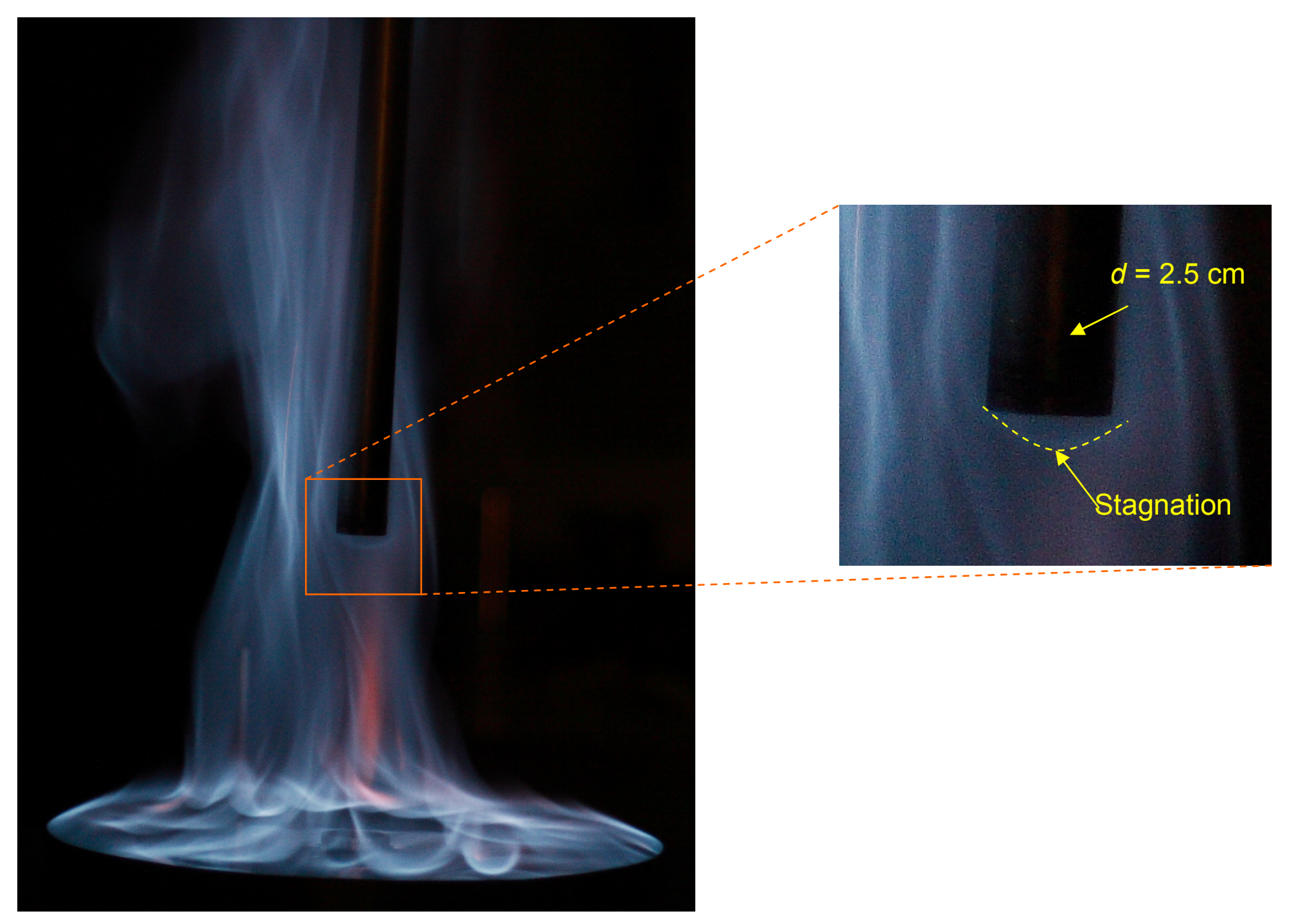

Figure 3.16: Photograph of $d=30 \mathrm{~cm}$ methanol pool fire test at probe height $z=15 \mathrm{~cm}$, illustrating probe effects 


\section{CHAPTER 4: Calculation of Species Concentrations}

The concentrations of combustion species inside a $d=30 \mathrm{~cm}$ methanol pool fire were evaluated by fitting experimentally measured infrared absorbance spectra to modelderived spectra calculated from an integrated radiation-transport model using temperature and concentration fields for the fuel-rich core of the flame. The model was developed in MATLAB for calculating theoretical spectra by solving the radiation transport equation through the fuel-rich flame core by using temperature-dependent absorption coefficients $\mathrm{CH}_{3} \mathrm{OH}, \mathrm{H}_{2} \mathrm{O}, \mathrm{CO}$, and $\mathrm{CO}_{2}$. The absorption coefficients used in the model, $\kappa_{v, k}^{\prime}$ as described in eqs. (1.21) to (1.26), were obtained from the study by Wakatsuki. ${ }^{17}$ Temperature profiles for the fuel-rich flame core presented in Chapter 3 were used. The MATLAB model solved for an optimal species concentration (or molefraction) profile using a constrained gradient-based optimization function to fit the model-calculated absorbance spectra at various heights to those measured in the flame experiments.

\subsection{Description of the concentration profile solver}

The MATLAB-based code to solve for concentration profiles by fitting the experimental absorbance spectra consists of two critical parts. A numerical function is programmed to calculate radiative transport and thus absorbance along a line for $T, X_{k}$, and $P$ profiles along a path-line using tabulated $\kappa_{k}^{\prime}$, which are functions of temperature. ${ }^{17}$ This function is integrated into a MATLAB-based code that utilizes an optimization 
algorithm to solve for species mole fractions that yield predicted theoretical absorbance spectra that most closely match the measured spectra. The code calls the radiative transport function and iteratively solves for the best species mole fractions $X_{k}$ to fit selected absorbance spectra at various heights in the fuel-rich flame core.

In the current study, the calculated transport is being compared to FT-IR spectra measured in "transmission mode," and emission of gases along the pathlength is not considered in the transport equations. Thus, the 1-D integration only considers absorption in the transport equation and takes into account the dependence of absorption coefficients on both $T$ and $P_{k}$.

In the experimental measurements, the FT-IR source enters the fuel-rich flame core from underneath the pool surface by passing through an $\mathrm{N}_{2}$-purged passageway through the pool. Thus, the transport equations are solved by starting at the pool surface $(z=0 \mathrm{~m})$ with a zero absorbance $(A=0)$ and integrating the radiative transport equation over discretized paths up to a selected experimental measurement point at some location $z_{\text {meas. }}$ This gives a pathlength of $z_{\text {meas,max }}$, which is divided into small increments of $\Delta z \leq 1$ $\mathrm{cm}$ for the numerical integration. The absorbance contributed by each species $k$, is summed for $k_{t o t}$ species into the same spectral line:

$$
\begin{gathered}
\Delta A_{v, \text { calc }}=\sum_{k=1}^{k_{\text {tot }}} d A_{v, k}=\sum_{k=1}^{k_{\text {top }}} \kappa_{v, k}^{\prime}(v, T) P_{k}(z) \Delta z \\
A_{v, \text { calc }}(L)=\sum_{z(i)=0}^{z_{\text {meas }}} \Delta z_{i} \sum_{k=1}^{k_{\text {tor }}} \kappa_{k}^{\prime}(v, T) P_{k}(z)
\end{gathered}
$$

Alternately, this equation can be represented more simply with matrix algebra:

$$
\mathbf{P}_{z}=P_{1}(z) \quad \cdots \quad P_{k_{t o t}}
$$




$$
\begin{aligned}
& \kappa_{1}^{\prime}(v, T) \\
& \mathbf{K}_{v}=\quad \vdots \\
& \kappa_{k_{t o t}}^{\prime}(v, T) \\
& A_{v, \text { calc }}(L)=\sum_{z(i)=0}^{z_{\text {meas }}} \mathbf{P}_{z} \mathbf{K}_{v} \Delta z_{i}
\end{aligned}
$$

The result of eq. (4.5) can then be compared to the experimental result at each height $z_{\text {meas }}$ where there is a measurement up to the maximum height selected $z_{\text {meas,max. }}$ An algorithm was devised to calculate the residual, $S$, of a least-squares fit between the measured absorbance and calculated absorbance spectra:

$$
\begin{gathered}
S=\sum_{z(i)}^{z(n)} \sum_{V_{1}}^{v_{n}}\left(1-\frac{A_{v, \text { meas }}(z(i+1))-A_{v, \text { meas }}(z(i))}{A_{v, \text { calc }}(z(i+1))-A_{v, \text { calc }}(z(i))}\right)^{2} \forall \Delta A_{v, \text { meas }}(z)>A_{\text {min }}, \\
z(i)=5,10,15,20 \mathrm{~cm}
\end{gathered}
$$

The condition of a minimum difference recorded in the absorbance at two consecutive heights is required to prevent dividing by zero and to avoid trying to fit calculated profiles to noise in the experimental data. However, $A_{\min }$ may also be adjusted to focus fits on different zones of the absorption bands. A comparison of the resulting fits by varying $A_{\min }$ is discussed in section 4.2. Minimization of $S$ in Eq. (4.6) was solved with the MATLAB function 'fmincon' by varying for species mole faction profiles $X_{k}$ (= $P_{k}\left(P_{\mathrm{tot}}\right) . \quad S$ is generally calculated by summing line-by-line over user-selected spectral bands for all heights of interest. For example, an analysis of the structure of $\mathrm{CO}$ and $\mathrm{CO}_{2}$ through the centerline of the fire would compare $A_{\text {calc }}$ and $A_{\text {meas }}$ between 2000 and 2400 $\mathrm{cm}^{-1}$, which is sufficiently broad to capture the overlapping $\mathrm{CO}_{2}$ and $\mathrm{CO}$ bands centered 
at $2349 \mathrm{~cm}^{-1}$ and $2143 \mathrm{~cm}^{-1}$, respectively. No other bands provided clear data for $\mathrm{CO}_{2}$ in the FT-IR spectra presented in Chapter 3. Calculating $S$ for $\Delta v$ larger than required needlessly adds to the computation time. Table 4.1 lists the bands used in the current study for analysis of the particular species of interest.

An initial guess of the concentration profile is provided to the solver along $1 \mathrm{~cm}$ increments across the height of the computed domain. The solver calculates the steplength to be the minimum of a selected $\Delta z_{\min }$ or $1 / \kappa_{\nu}$ predicted by the numerical model, given local values of $P_{k}, T$, and $\kappa_{v, k}^{\prime} . \Delta z_{\min }$ is usually set at $0.01 \mathrm{~m}$ although some fits were run at $0.005 \mathrm{~m}$ with minimal change in the fits.

The residual is calculated and MATLAB function 'fmincon' iteratively changes the concentration profiles until the value of $S$ converges to a local and hopefully near global minimum. The function allows the user to constrain the solution. Constraints can include user-specified minimum and maximum $X_{k}$, and, if known, the concentration can be forced to follow user-specified functionalities along a particular profile. For example, a constraint forcing $\mathrm{CH}_{3} \mathrm{OH}$ concentration to decrease as $z$ increases would be logical because the methanol source is at the pool surface, $z=0$, and is subsequently consumed at the flame boundary. This constraint is applied with the caveat that it does not apply to the first data point in order to allow the code to adjust for the effects of the cone protruding above the fuel surface and its $\mathrm{N}_{2}$ purge. To add stability to the solutions for $\mathrm{CO}_{2}$ and $\mathrm{CO}$ profiles, a maximum volume fraction, $X_{\mathrm{k}, \max }$, of 0.50 was used, which was more than double the maximum concentrations recorded by a gas chromatograph (GC), reported in Chapter 3. A list of constraints used is provided in Table 4.1. 
Table 4.1: Table of infrared bands used for species analysis of methanol pool fire

\begin{tabular}{|c|c|c|c|c|c|}
\hline & $\begin{array}{c}\begin{array}{c}\text { Vibration } \\
\text { mode }\end{array} \\
\end{array}$ & $\begin{array}{l}\text { Central } \\
\text { peak, } v_{0}\end{array}$ & Band & Constraint(s) & Remarks \\
\hline $\mathrm{CH}_{3} \mathrm{OH}$ & $\begin{array}{c}\mathrm{C}-\mathrm{O} \\
\text { stretching }\end{array}$ & $1033 \mathrm{~cm}^{-1}$ & $\begin{array}{l}1000 \text { to } \\
1100 \mathrm{~cm}^{-1}\end{array}$ & $\begin{array}{c}\left(X_{\min }, X_{\max }\right)=(0,1) \\
X \text { decreases with } \\
z>1 \mathrm{~cm}\end{array}$ & $\begin{array}{l}\kappa_{v}^{\prime} \text { noisy } \\
\text { at high } T\end{array}$ \\
\hline $\mathrm{CH}_{3} \mathrm{OH}$ & $\begin{array}{c}\mathrm{C}-\mathrm{H} \\
\text { stretching }\end{array}$ & $\begin{array}{l}2844 \text { and } \\
2981 \mathrm{~cm}^{-1}\end{array}$ & $\begin{array}{l}2800 \text { to } \\
3100 \mathrm{~cm}^{-1}\end{array}$ & $\begin{array}{c}\left(X_{\min }, X_{\max }\right)=(0,1) \\
X \text { decreases with } \\
z>1 \mathrm{~cm}\end{array}$ & \\
\hline $\mathrm{CO}_{2}$ & $\begin{array}{c}\text { Asymmetric } \\
\text { stretching }\end{array}$ & $2349 \mathrm{~cm}^{-1}$ & $\begin{array}{c}2150 \text { to } \\
2400 \mathrm{~cm}^{-1}\end{array}$ & $\begin{array}{l}\left(X_{\min }, X_{\max }\right)=(0,0.2) \\
\left|\Delta X_{\max }\right|=0.03\end{array}$ & $\begin{array}{l}\text { CO band } \\
\text { overlap }\end{array}$ \\
\hline $\mathrm{CO}$ & Stretching & $2143 \mathrm{~cm}^{-1}$ & $\begin{array}{c}2000 \text { to } \\
2250 \mathrm{~cm}^{-1}\end{array}$ & $\begin{array}{l}\left(X_{\text {min }}, X_{\text {max }}\right)=(0,0.2) \\
\Delta X_{\text {max }} \mid=0.03\end{array}$ & $\begin{array}{c}\mathrm{CO}_{2} \text { band } \\
\text { overlap }\end{array}$ \\
\hline
\end{tabular}

In the case of this study, experimental data recorded at $0.5 \mathrm{~cm}^{-1}$ resolution is being compared to absorption coefficients measured at $1 \mathrm{~cm}^{-1}$ resolution. Both resolutions are sufficiently fine to capture most individual peaks. Thus, a cubic spline is used to fit the experimental results onto the lower resolution recorded in Wakatsuki's absorption database. ${ }^{17}$ The spline fit created a generally accurate representation of the peak shapes. Sometimes peaks and troughs were not accurately captured, but the effect of one or two lines on an entire band is small.

Several features of the optimization program have been explored. First, results using different possible $T$ profiles were compared. Since infrared absorption is highly temperature dependent, it was expected that the results would be noticeably different. Another consideration was whether using different wavenumber regions made a difference in the model fits. Also, the sensitivity of the result to the value of $A_{\min }$ was investigated. 


\subsection{Results of the concentration profile solver}

\subsubsection{Methanol $\left(\mathrm{CH}_{3} \mathrm{OH}\right)$}

Absorbance spectra measured at heights of 5, 10, 15, and $20 \mathrm{~cm}$ above the pool surface in the $\mathrm{CH}_{3} \mathrm{OH}$ fire were used to fit predicted absorption spectra. $\mathrm{CH}_{3} \mathrm{OH}$ mole fractions were first calculated by fitting the C-O stretching band over the wavenumber range between 900 and $1100 \mathrm{~cm}^{-1}$. The $\mathrm{C}-\mathrm{O}$ stretching band also has the strongest absorption of all $\mathrm{CH}_{3} \mathrm{OH}$ bands and therefore provides the highest sensitivity to fits for $X_{\mathrm{CH} 3 \mathrm{OH}}$. Furthermore, this band does not overlap any of the major combustion species bands or any of the hydrocarbon intermediates found in the flame. However, Wakatsuki's database of temperature-dependent absorption coefficients of $\mathrm{CH}_{3} \mathrm{OH}$ had a high level of noise below $1000 \mathrm{~cm}^{-1}$ for high temperatures, as shown in Figure 4.1. Therefore, this band was fitted only using the 1000 to $1100 \mathrm{~cm}^{-1}$ wavenumber region. $A_{\text {min }}$ is initially set to 0.01 as another method to limit the impact of noise on the fitting function; consequences of changing this parameter are discussed later in the section. The 


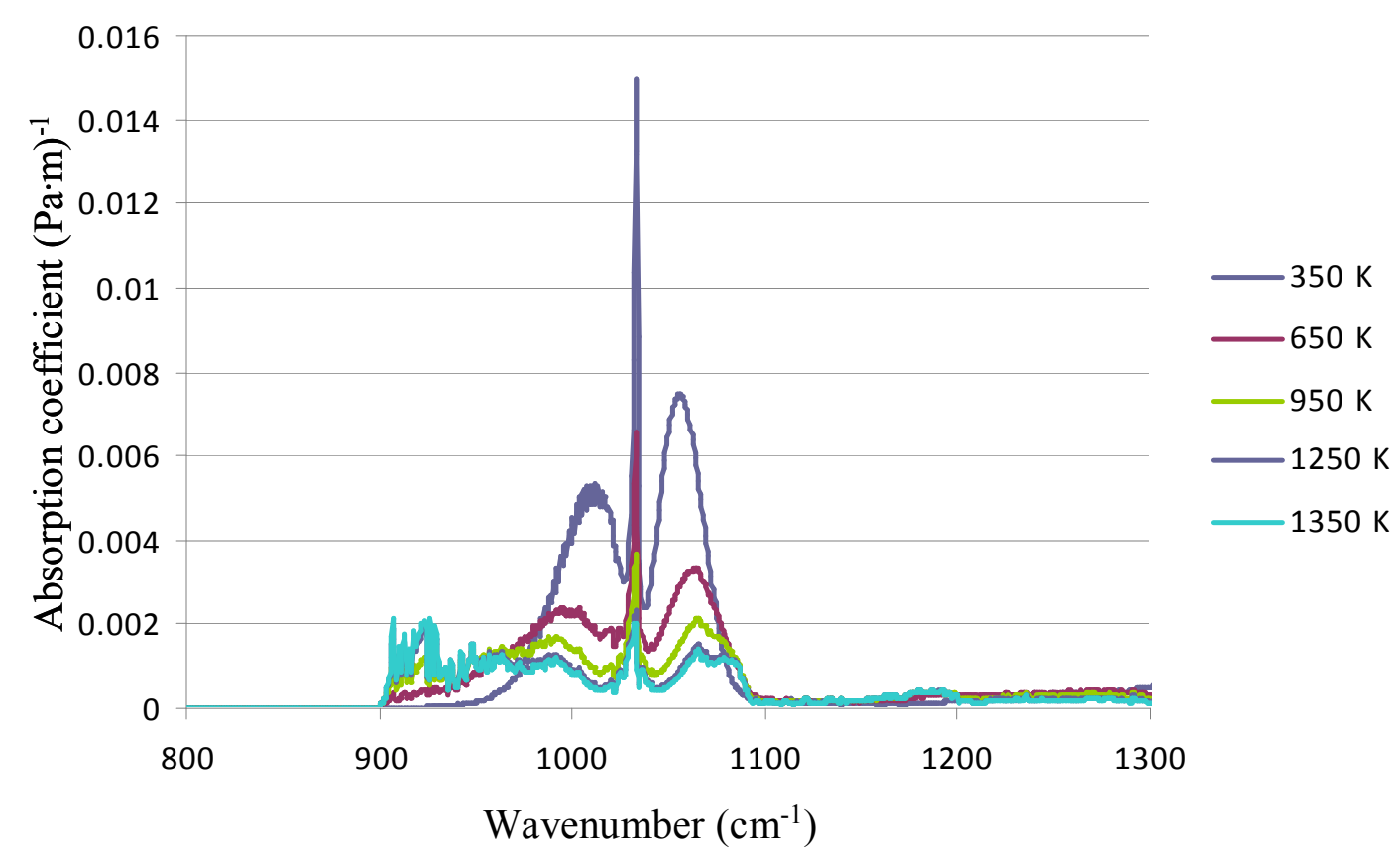

Figure 4.1: Temperature-dependent absorption coefficients for $\mathrm{CH}_{3} \mathrm{OH}$ used in concentration fit study

less noisy 2800 to $3100 \mathrm{~cm}^{-1}$ region encompassing the $\mathrm{C}-\mathrm{H}$ stretching peaks for $\mathrm{CH}_{3} \mathrm{OH}$ was also explored, though this band was not initially preferable due to the absorbance of other hydrocarbons in this region. However, the GC concentration results show that there is only a trace amount of other absorbing compounds in the plume, and their spectral signature is not expected to greatly affect the $\mathrm{CH}_{3} \mathrm{OH}$ fits.

In fitting the $X_{\mathrm{CH} 3 \mathrm{OH}}$ centerline profile, it became clear that the fits were highly sensitive to the assumed temperature profile, particularly near the flame surface. This is particularly the case for the $X_{\mathrm{CH} 3 \mathrm{OH}}$ profile because of the high concentrations near the pool surface and the steep temperature gradients in the gas-phase near the pool surface. Although temperatures were measured for a range of heights in this study as discussed in Chapter 3, the resolution and accuracy of those measurements particularly near the pool 
surface were not adequate for them to provide the full temperature profile for the fits based on equation 4.6. Thus, other corroborating sources of temperature profiles were sought for the $30 \mathrm{~cm}$ diameter $\mathrm{CH}_{3} \mathrm{OH}$ pool fire.

To build an adequate centerline temperature profile, data points from the present study, Weckman and Strong, ${ }^{21}$ and Hamins and Gorchkov ${ }^{22}$ describing the vertical centerline of a $d=30 \mathrm{~cm}$ pool fire were interpolated and extrapolated linearly across the experimental domain, which produces profiles similar to the ones shown in Figure 3.10. These profiles are shown in Figure 4.2. Because of its high concentration near the surface, only $\mathrm{CH}_{3} \mathrm{OH}$ spectra were a useful tool for determining the effectiveness of the temperature fit in the steep-gradient region near the pool surface. The normalized concentration profile of methanol measured by the GC and provided in Chapter 3 was used to model the absorbance spectra at the experimental heights. Figure 4.3 shows a comparison between the measured and calculated spectra at the experimental heights $z$ for the various temperature profiles. At this point, the optimization program is not being utilized; the only goal is to evaluate how temperature profiles affect band shapes by using a generic data set. 


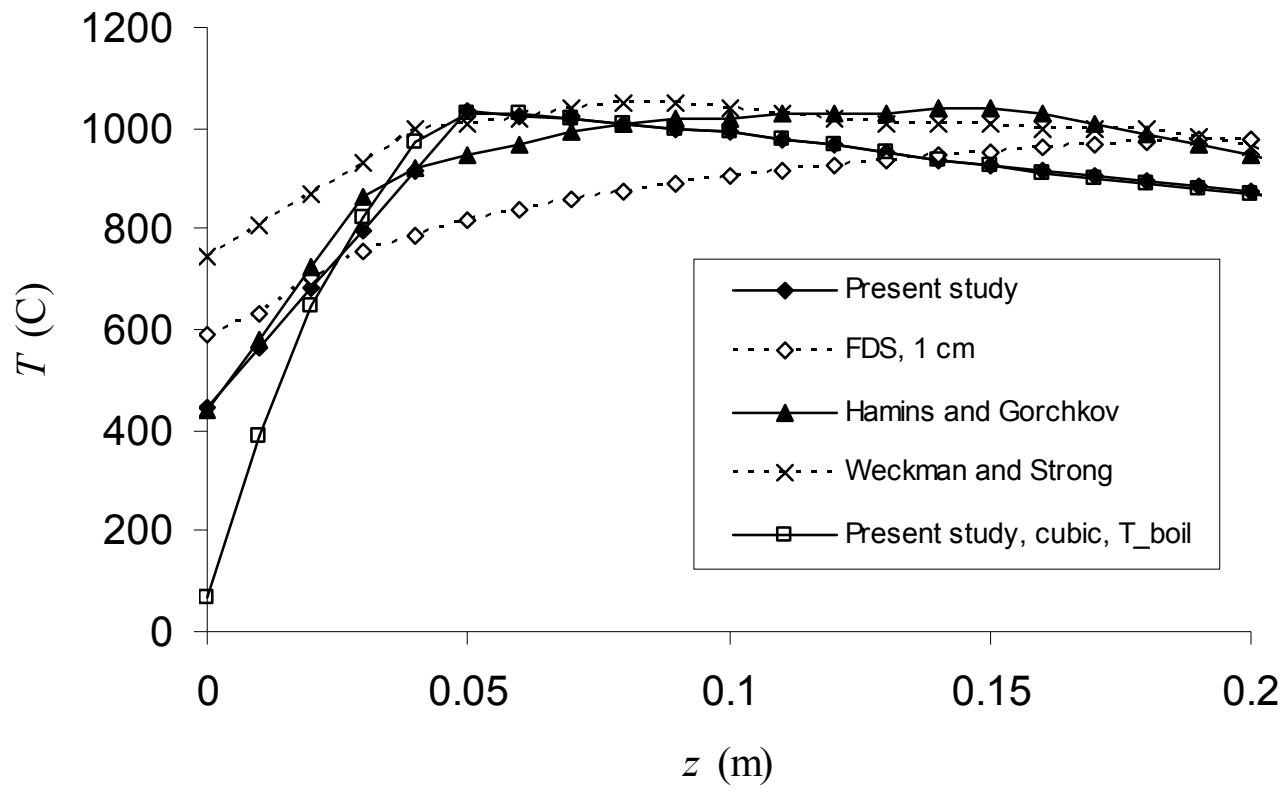

Figure 4.2: Linearly interpolated experimental temperature profiles and pool surface conditioned cubic temperature fit used by spectra calculator for comparison to experimental spectra 

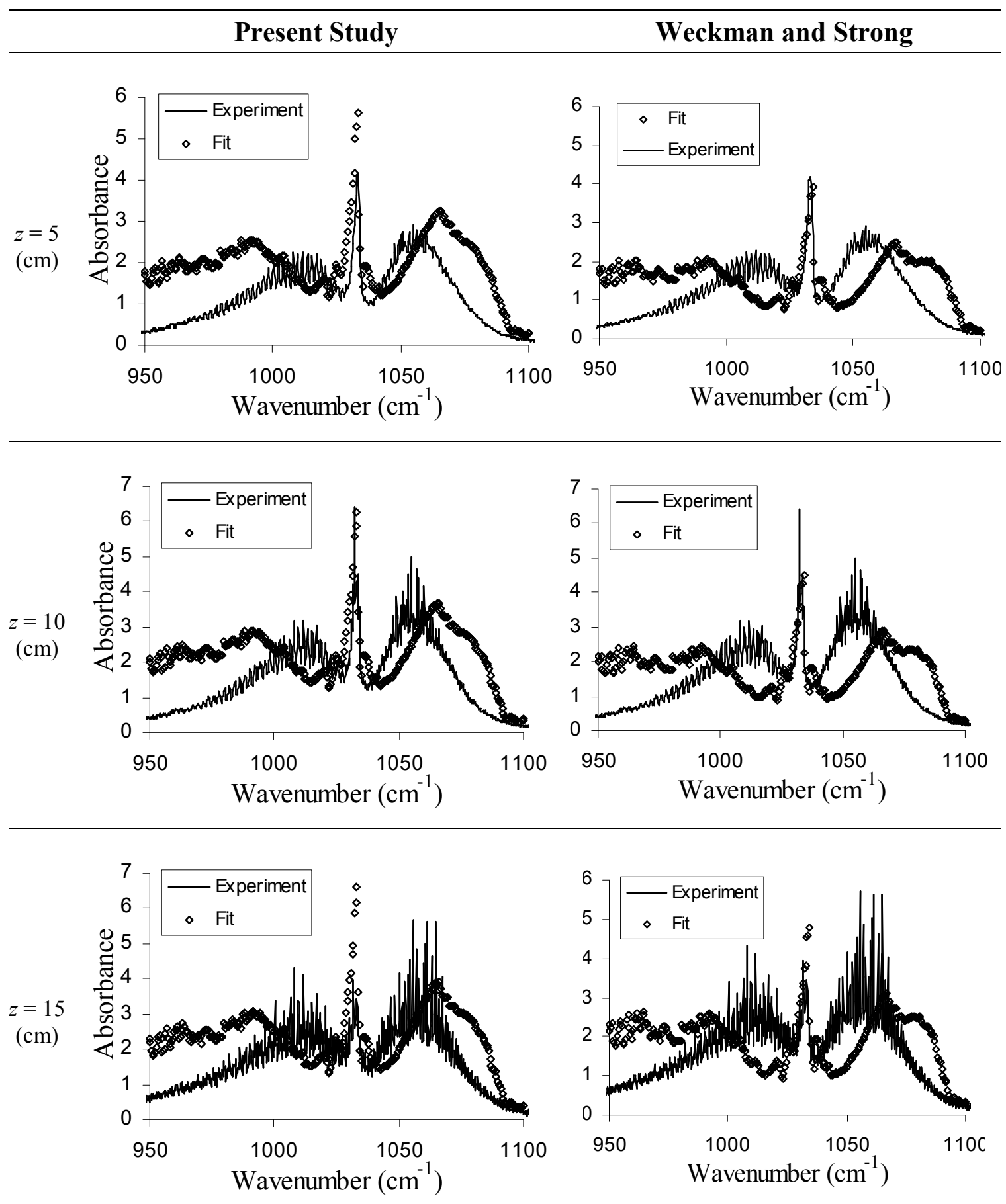

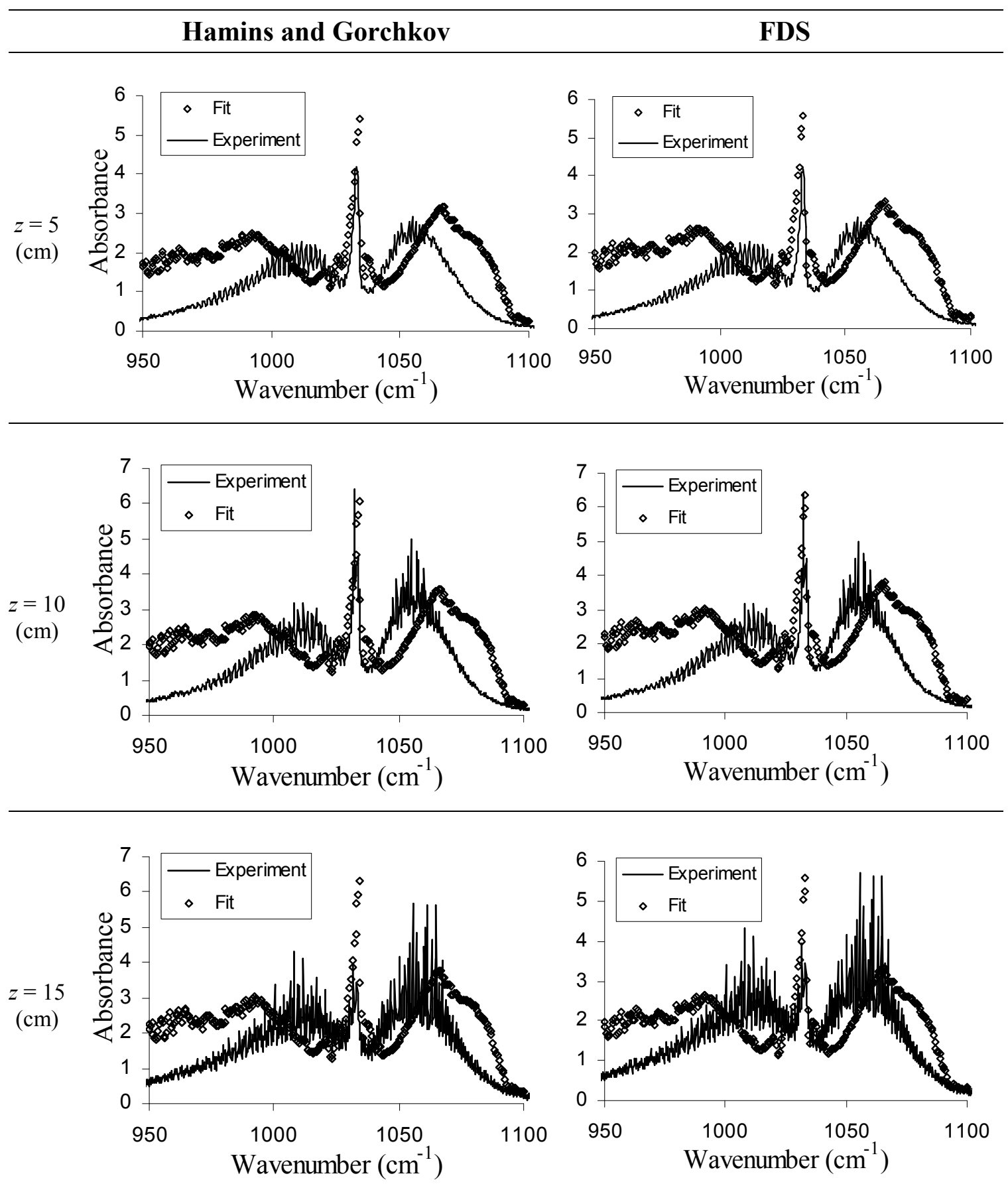

Figure 4.3: Calculated spectra fits in methanol C-O stretching band using $\mathrm{CH}_{3} \mathrm{OH}$ concentration profile from GC measurements with temperatures from the Present study, Weckman and Strong, Hamins and Gorchkov and FDS direct numerical simulation result solved with $1 \mathrm{~cm}$ grid, linearly extrapolated 
Two band features systematically affected by temperature are the line absorption strength and the bandwidth. As $T$ increases, the absorption weakens and band grows wider. Of the various temperature profiles used, the present study has the lowest temperatures near the fuel surface. As a result, the absorbance spectra calculated using this temperature profile is the strongest and narrowest of all the temperature cases. Low temperature creates the largest difference of magnitude between theory and experiment, but provides the best qualitative band shape. One manner the band shapes may be qualified is by remarking the frequencies at which the $\mathrm{P}$ and $\mathrm{R}$ wings of the absorbance band reach their maximum amplitudes, and how they compare to the experimental spectra. This correlation is directly related to the temperature of the absorbing compound because the distribution of rotational energy states is defined by a Boltzmann distribution. ${ }^{30}$ As the optimization solver solves for its own concentrations and exhibits tendencies to correct for the magnitude of absorbance more easily than the band shape, it was preferred to use the present study's temperature profile for most of the concentration fits for this study.

Temperatures near the surface are crucial to the $\mathrm{CH}_{3} \mathrm{OH}$ absorption spectra because of the strong absorption at the low temperatures and high concentrations of $\mathrm{CH}_{3} \mathrm{OH}$ near the pool surface. The calculated spectra are broader than the experimental, suggesting that the temperature near the pool surface is overestimated. The lack of accurate, high spatial resolution temperature data in this region creates difficulty in predicting adequate fits. Some hypotheses regarding the temperature profile were tested by comparing the difference made in absorbance spectra. One hypothesis providing a good fit to experimental spectra was to set $T$ at the fuel surface equal to the boiling 
temperature of methanol $\left(T_{\text {boiling }}=65^{\circ} \mathrm{C}\right)$ and to more accurately account for the rapid $T$ increase above the surface by using a cubic fit. This temperature profile is shown in Figure 4.2. This change was justified because the temperature at the surface is theoretically expected to be around the boiling point and the thermocouple measurements are not adequate at resolving the rapid temperature drop to $T_{\text {boiling. }}$ Furthermore, the presence of the stainless steel cone in the center of the pool pan can further contribute to the cool temperatures along the centerline near the pool surface.

A computed fit of the spectra for the methanol C-O stretching band, using the current study's $T$ measurements on a cubic interpolation with $T(0)=T_{\text {boiling, }}$ produced the mole fraction profile shown in measurements in Figure 4.4, the fitted spectra are shown in Figure 4.5. A comparison between these figures shows that a cooler surface temperature creates a better fit to experimental spectra, but the predicted profiles are substantially different from the GC measurement profiles.

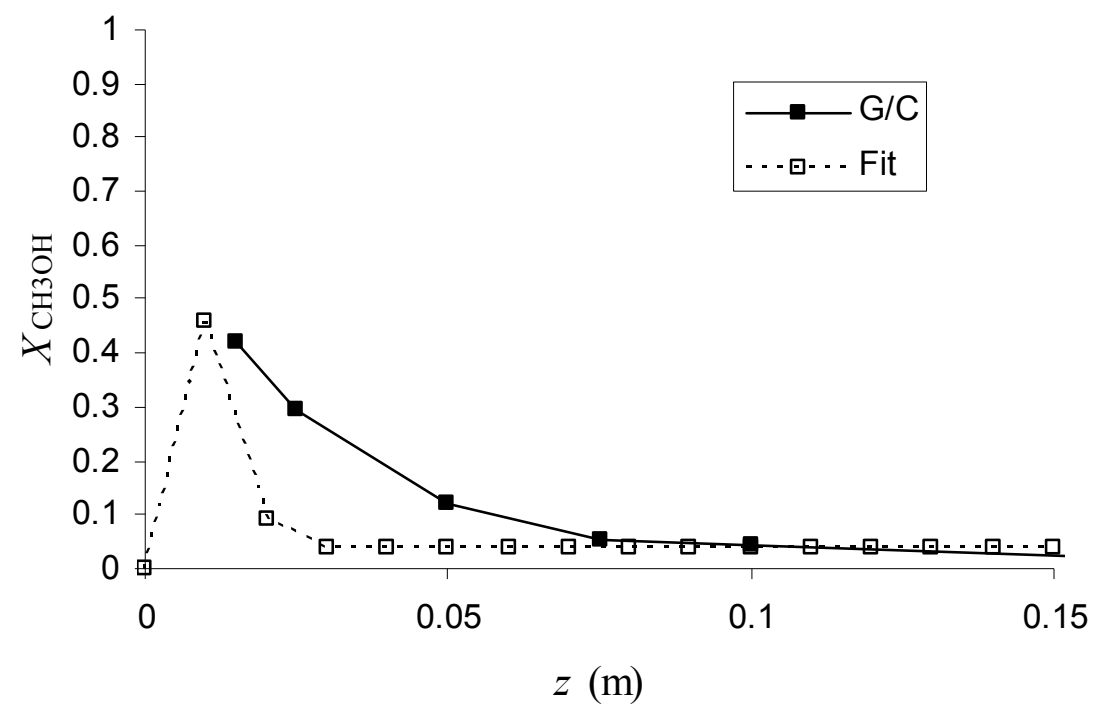

Figure 4.4: Predicted $\mathrm{CH}_{3} \mathrm{OH}$ mole fraction profile when fitted to experimental spectra with surface-conditioned cubic temperature profile 
$z=5 \mathrm{~cm}$

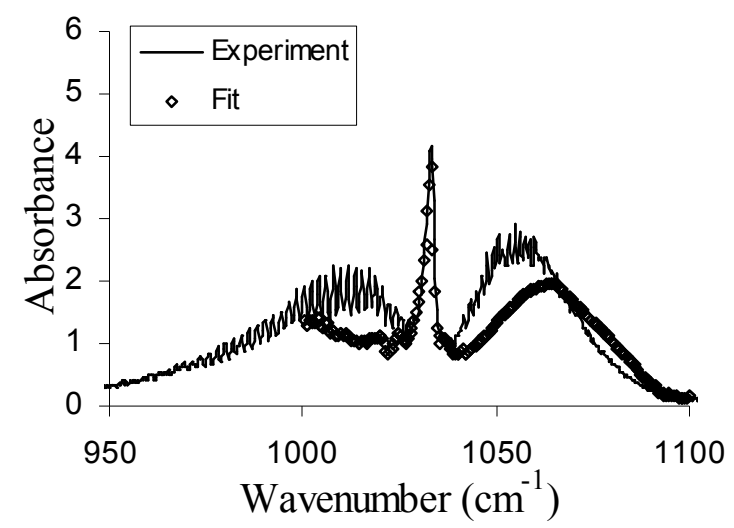

$z=10 \mathrm{~cm}$

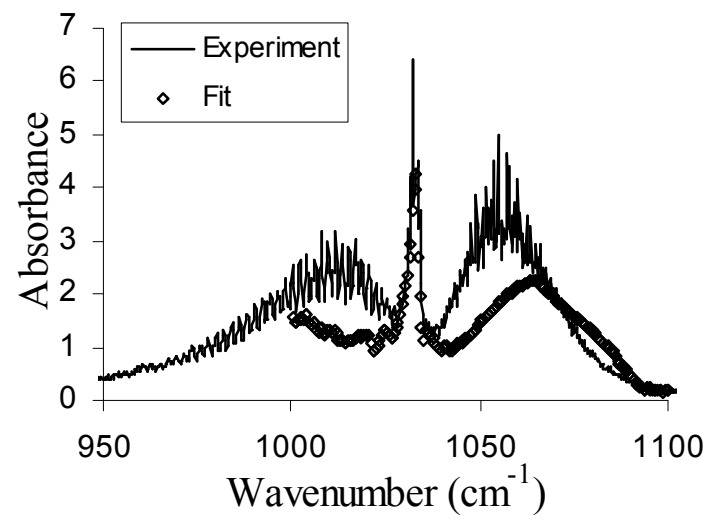

$z=15 \mathrm{~cm}$

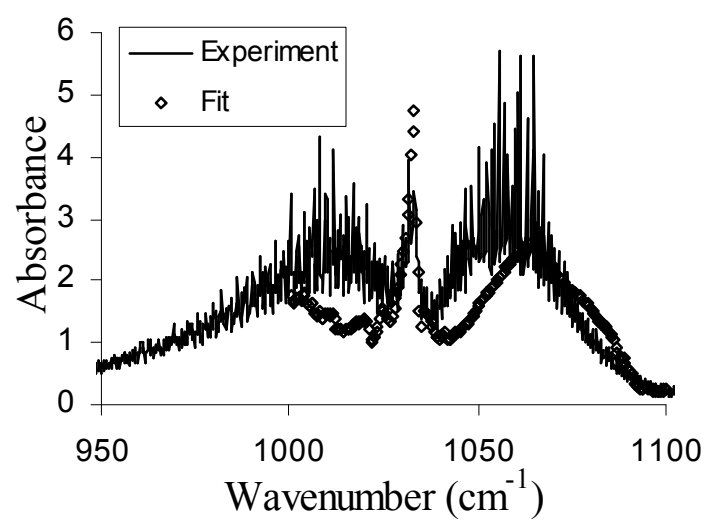

Figure 4.5: $\mathrm{CH}_{3} \mathrm{OH}$ spectra fits to experiment using temperature profile from the present study with $T(0)=T_{\text {boiling }}$ conditioned fit

These results show that the calculated fits for mole fraction can significantly improve the fits to the experimental spectra, particularly along the wings. However, the mole fraction fit does not resemble the experimental concentrations measured with a GC. 
A persistent problem was fitting logical concentration profiles at $1 \mathrm{~cm}$ resolution in the region of the centerline above $z=5 \mathrm{~cm}$. In the case where concentrations were constrained to decay in a particular direction, the fitted concentrations in this region were typically flat. In the non-constrained cases, the fitted concentrations in this region were randomly noisy. The reason this does not occur in the near fuel surface region is because there is a sharp gradient of temperature which greatly affects the shape of the resulting calculated spectra. Above that zone, the temperature is much flatter and the code simply does not have enough information to produce a fit at this high resolution. In order to capture fits at a small spatial resolution, experimental spectra must be recorded at smaller $\Delta z$. The experimental result for the methanol concentration profile from the GC measurements provides some insight onto how constraints can be applied to determine a better fit to experiment. Instead of using a constraint only stating that methanol must decrease by any absolute value, a relative constraint was added forcing methanol concentration to decrease to at least $80 \%$ of its value after every centimeter in order to force a decaying profile similar to one observed by the GC. This forces the solver to compensate for smaller fitted concentrations higher in the flame with larger concentrations of $\mathrm{CH}_{3} \mathrm{OH}$ near the surface. The resulting fit of $X_{\mathrm{CH} 3 \mathrm{OH}}$, shown in Figure 4.6, is expectedly more similar to the experimental GC profile, though still quantitatively far from what would be expected. The fitted spectra shown in Figure 4.7 are very wellmatched to the central peaks and the wide wings. 


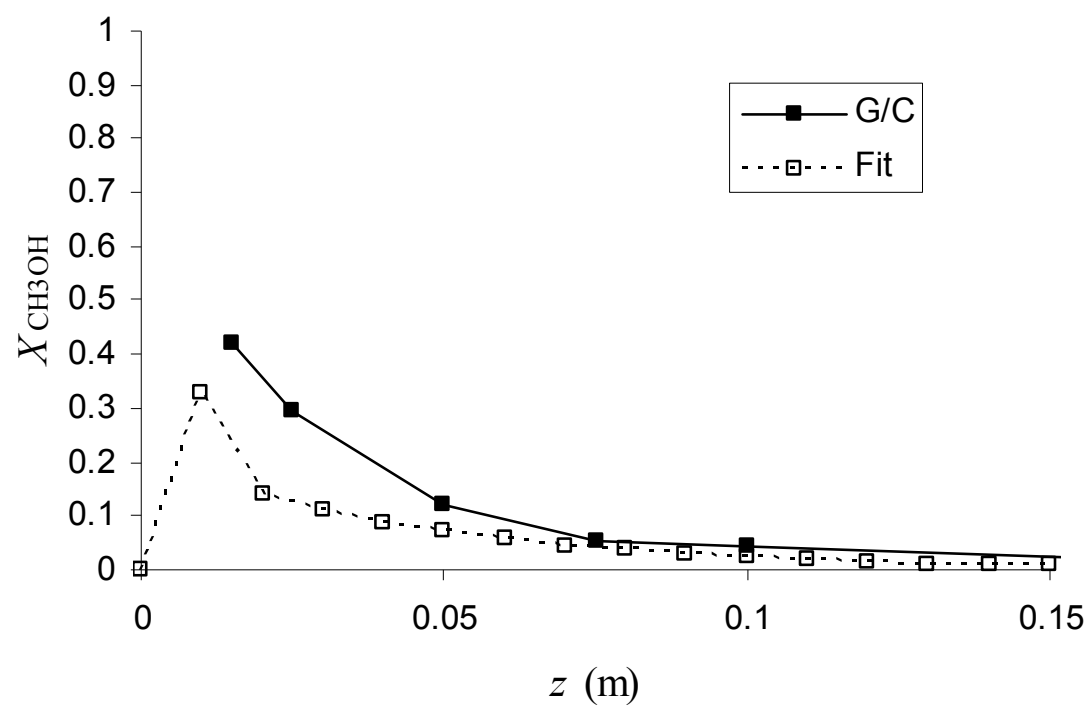

Figure 4.6: Predicted $\mathrm{CH}_{3} \mathrm{OH}$ mole fraction profile (forced decay to $<80 \%$ ) when fitted to experimental spectra with surface-conditioned cubic temperature profile 
$z=5 \mathrm{~cm}$

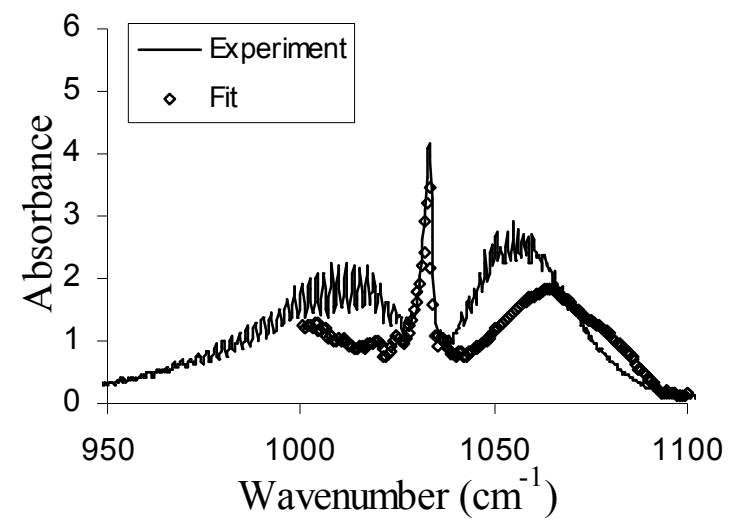

$z=10 \mathrm{~cm}$

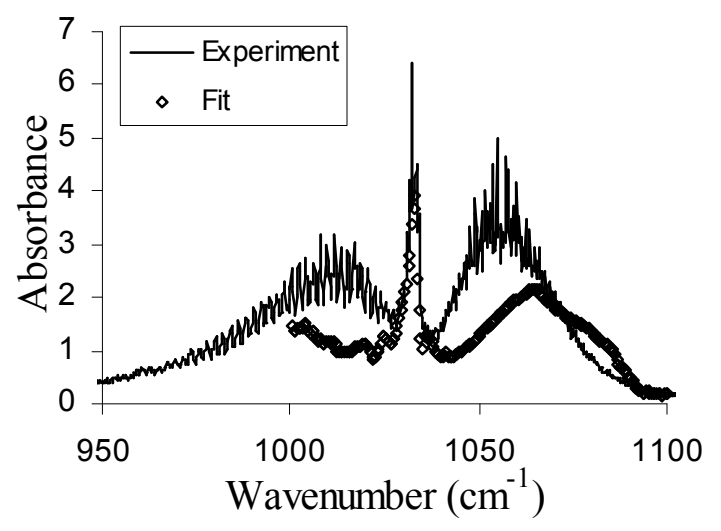

$z=15 \mathrm{~cm}$

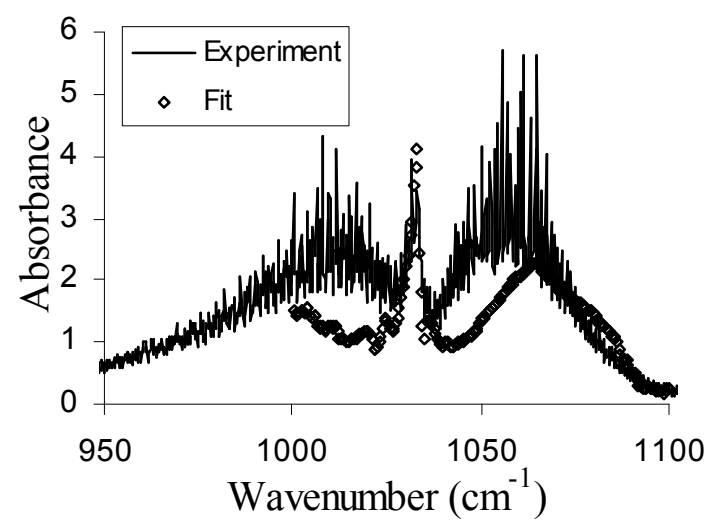

Figure 4.7: $\mathrm{CH}_{3} \mathrm{OH}$ spectra fits to experiment using temperature profile from the present study with $T(0)=T_{\text {boiling }}$ conditioned fit with concentration forced decay to $<80 \%$ 
Due to the noise in the absorption coefficients database for the C-O stretching band, the measurements were repeated using the C-H stretching bands from 2800 to $3100 \mathrm{~cm}^{-1}$. The two constraint conditions, the so-called "simple decay" constraint which allows for decay of any magnitude, and a forced $80 \%$ decay condition, are repeated for the fit. The concentration fits for these two constraints are compared to the GC experiment in Figure 4.8, while the predicted spectra in the $\mathrm{C}-\mathrm{H}$ band are compared to the experimental spectra for the two constraint cases in Figure 4.9. The resulting fits for mole fraction and absorbance spectra in the $\mathrm{C}-\mathrm{H}$ stretching band are closer to the experimental results as compared to fits performed in the C-O stretching band. Particularly, the $80 \%$ decay condition in this band provides a mole fraction fit which is very similar to the expected profile. However, the consequence of a lack of temperature data in the fuel-rich core still creates some problems when it comes to predicting the band shape, though the shape is qualitatively better than the fits for the C-O bands. It can be deduced from Figure 4.9 that the increased concentration of methanol downstream in the $80 \%$ decay case broadens the peak beyond the experimental result in the $3050-3100 \mathrm{~cm}^{-1}$ region.

Fitting in the $\mathrm{C}-\mathrm{H}$ band for methanol provides superior results, and this will be the band used in continuing analysis of the experiment and the MATLAB solver. 


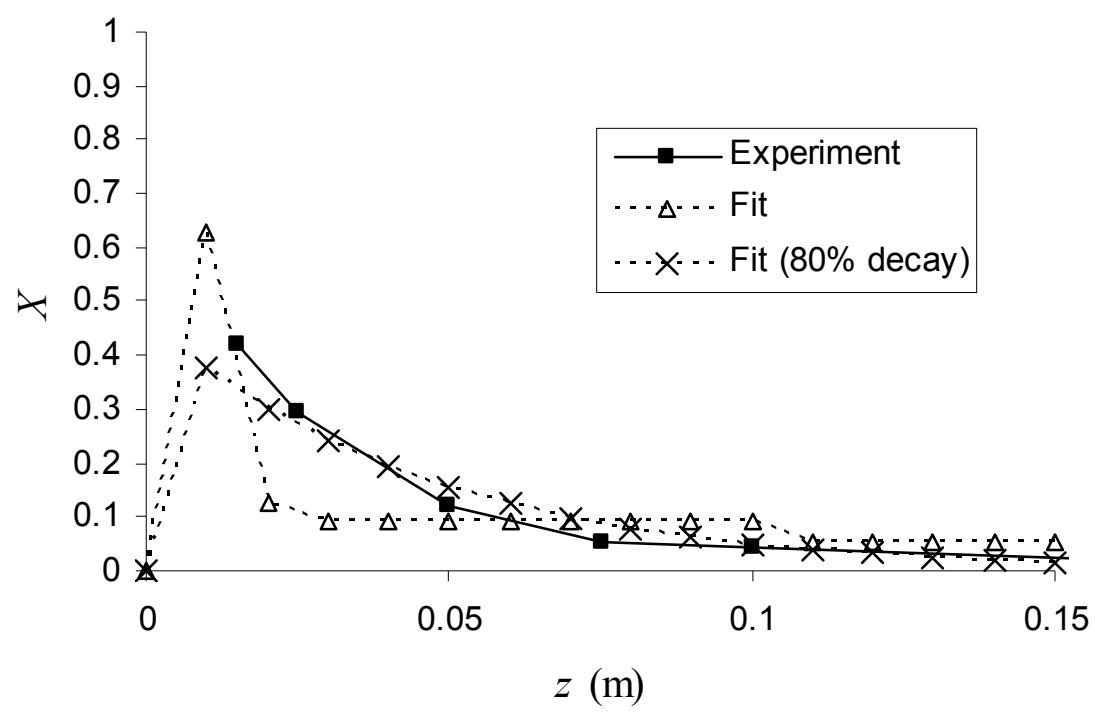

Figure 4.8: Predicted $\mathrm{CH}_{3} \mathrm{OH}$ mole fraction profile for two constraint cases when fitted to experimental spectra at $2800-3100 \mathrm{~cm}^{-1}$ (C-H stretching) 

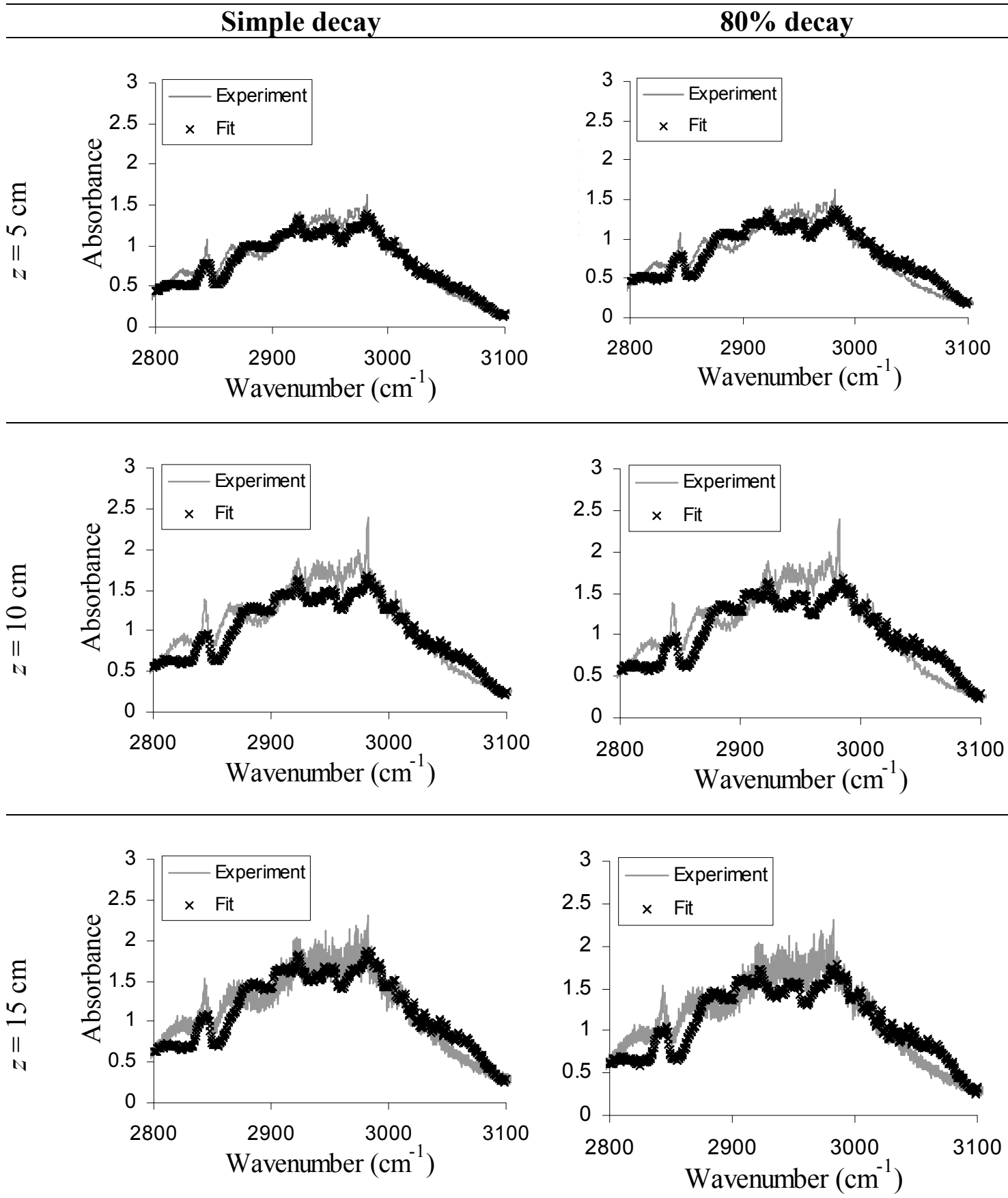

Figure 4.9: $\mathrm{CH}_{3} \mathrm{OH}$ spectra fit to experiment in $\mathrm{C}-\mathrm{H}$ stretching band with two different constraint models

The cool methanol vapor, found near the pool surface, absorbs in a relatively narrow band, similar to what is observed in the experimental spectra. Also, near the pool 
surface, methanol absorbs much more strongly than in hotter regions higher in the flame. When the solver creates a concentration fit in this region, it solves the correct band shape by choosing high concentrations where $T$ is relatively low. However, any slight overestimation of $X_{\mathrm{CH} 3 \mathrm{OH}}$ near the surface results in an underestimation of $X_{\mathrm{CH} 3 \mathrm{OH}}$ further from the pool surface. A forced decay in the profile somewhat corrects for this phenomenon. Small errors in temperature measurements can create large errors in this high $d T / d z$ region, and it is difficult to estimate the affect of the protruding cone piece on both the temperature and species concentration very near the pool surface. To analyze its possible effect on spectra shapes and mole fraction fits, it is assumed that the protrusion of the cone and the small $\mathrm{N}_{2}$ purge displaces the development of the temperature profile by $1 \mathrm{~cm}$, creating a modified temperature profile $\left(T_{\bmod }\right)$ shown in Figure 4.10 .

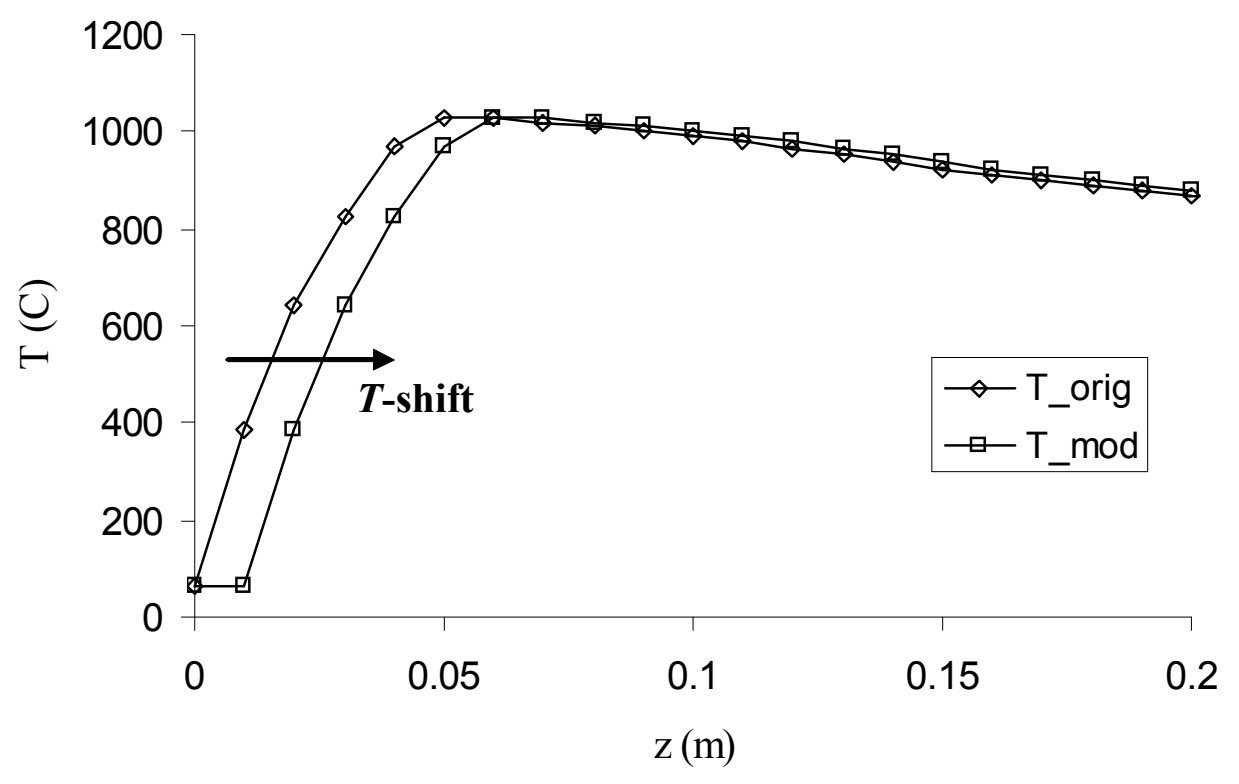

Figure 4.10: Modified temperature profile illustrating the assumed displacement of $T$ profile caused by stainless steel cone and $\mathrm{N}_{2}$ purge 
This shift, in effect, grows the low temperature region of the fuel-rich fuel core. The result of this fit will provide insight to the impact of the experimental setup on the temperature profiles. The results of the computed fit for the mole fraction of the two constraint methods ("Simple" and " $80 \%$ decay") using $T_{\bmod }$ are shown in Figure 4.11, and the predicted spectra are compared to the experimental data in Figure 4.12. The results show a considerable improvement upon the predicted band shape, while the predicted mole fractions are lower than fits recorded using the previous temperature profile. Both the narrowing band shape and the lower predicted concentrations are a result of the effect the lower core temperature has on reducing the line broadening in the absorption coefficients being read in by the calculator. Since the calculator is not able to change the temperature profile, it has a very limited ability to change the predicted band shapes. The improved band shape for this case in reference to the experiment is considered a success and justifies the continued use of the modified $T$ profile.

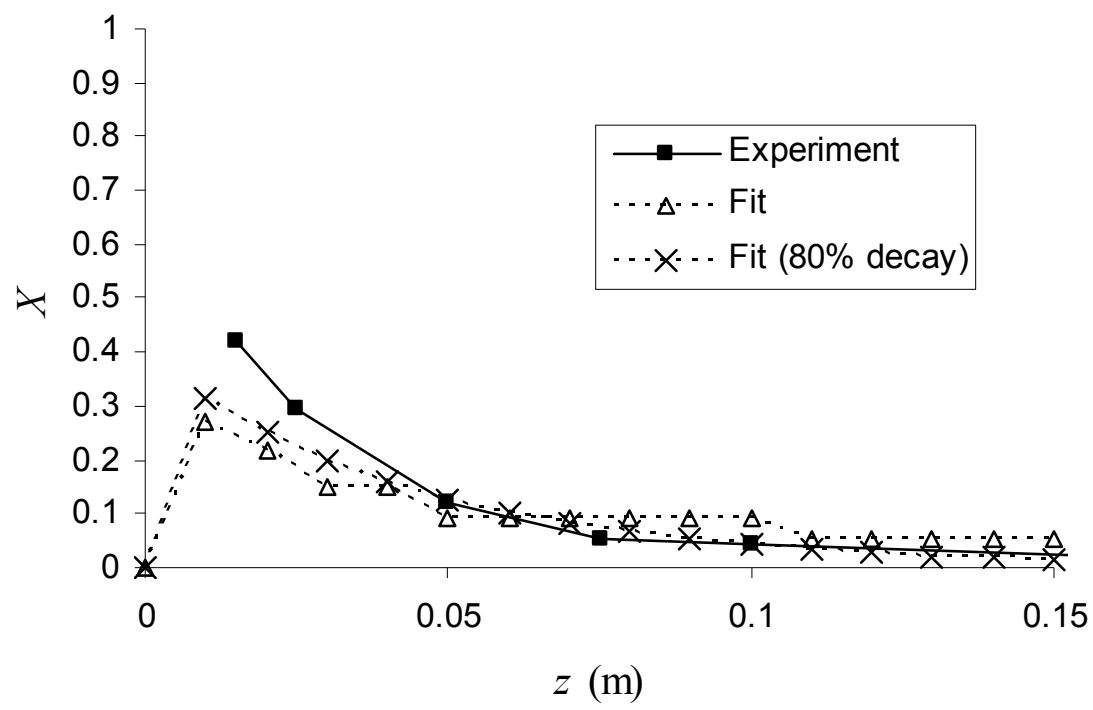

Figure 4.11: Predicted $\mathrm{CH}_{3} \mathrm{OH}$ mole fraction profile for two constraint cases when fitted to experimental spectra with a modified temperature profile, $T_{\bmod }$ 

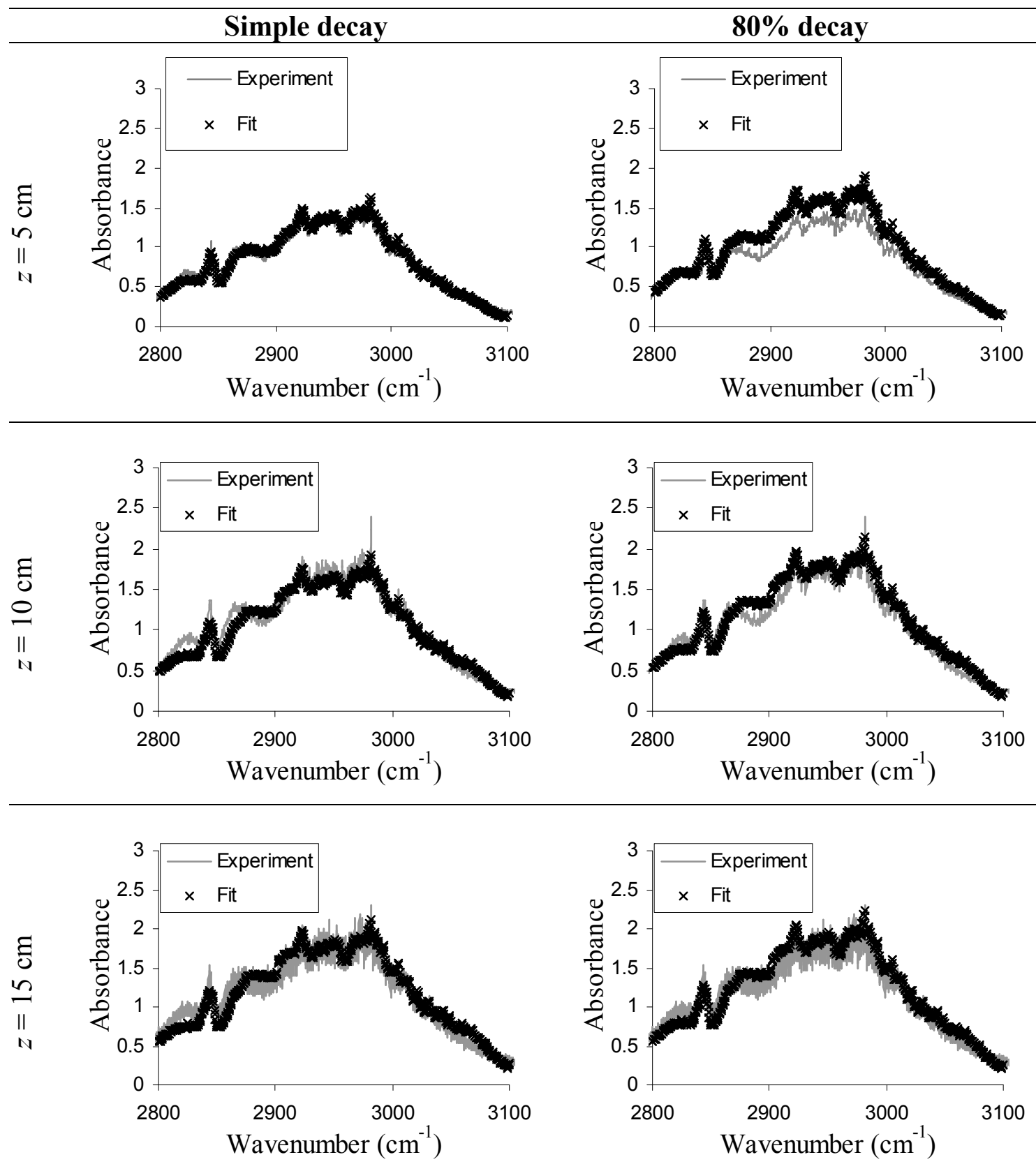

Figure 4.12: $\mathrm{CH}_{3} \mathrm{OH}$ spectra fits to experiment with two different constraint models using the modified temperature profile, $T_{\text {mod }}$

One parameter of the calculator, $A_{\min }$, has an effect on how the spectra are fitted in the temperature-broadened regions of the bands far from the central peak. The $\mathrm{C}-\mathrm{H}$ stretching band absorbances of $\mathrm{CH}_{3} \mathrm{OH}$ were fitted to the experimental spectra using, in 
addition to the initial setting of $A_{\min }=0.01, A_{\min }=0.005$ and 0.02 in order to develop a basis for how the solved absorbance fits trend when changing this parameter. This comparison was conducted in the $\mathrm{C}-\mathrm{H}$ stretching band using the modified $T$ profile with a constraint forcing $80 \%$ decay. A comparison of the mole fraction fit using the new values of $A_{\min }$ versus the initial setting is given in Figure 4.13. The resulting fits of predicted spectra to the measured spectra for the two cases are given in Figure 4.14. The results show that the fits are not sensitive to changes in $A_{\min }$ for fits involving this band of methanol.

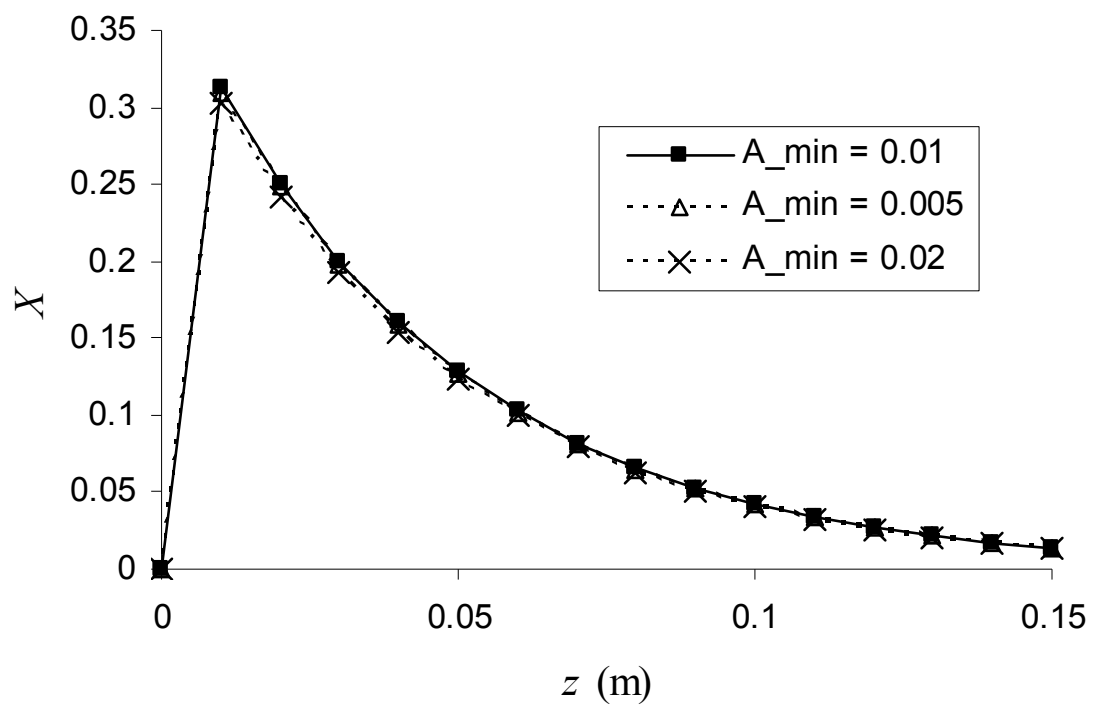

Figure 4.13: Comparison of $\mathrm{CH}_{3} \mathrm{OH}$ mole fraction fits using various values of $A_{\text {min }}$ 

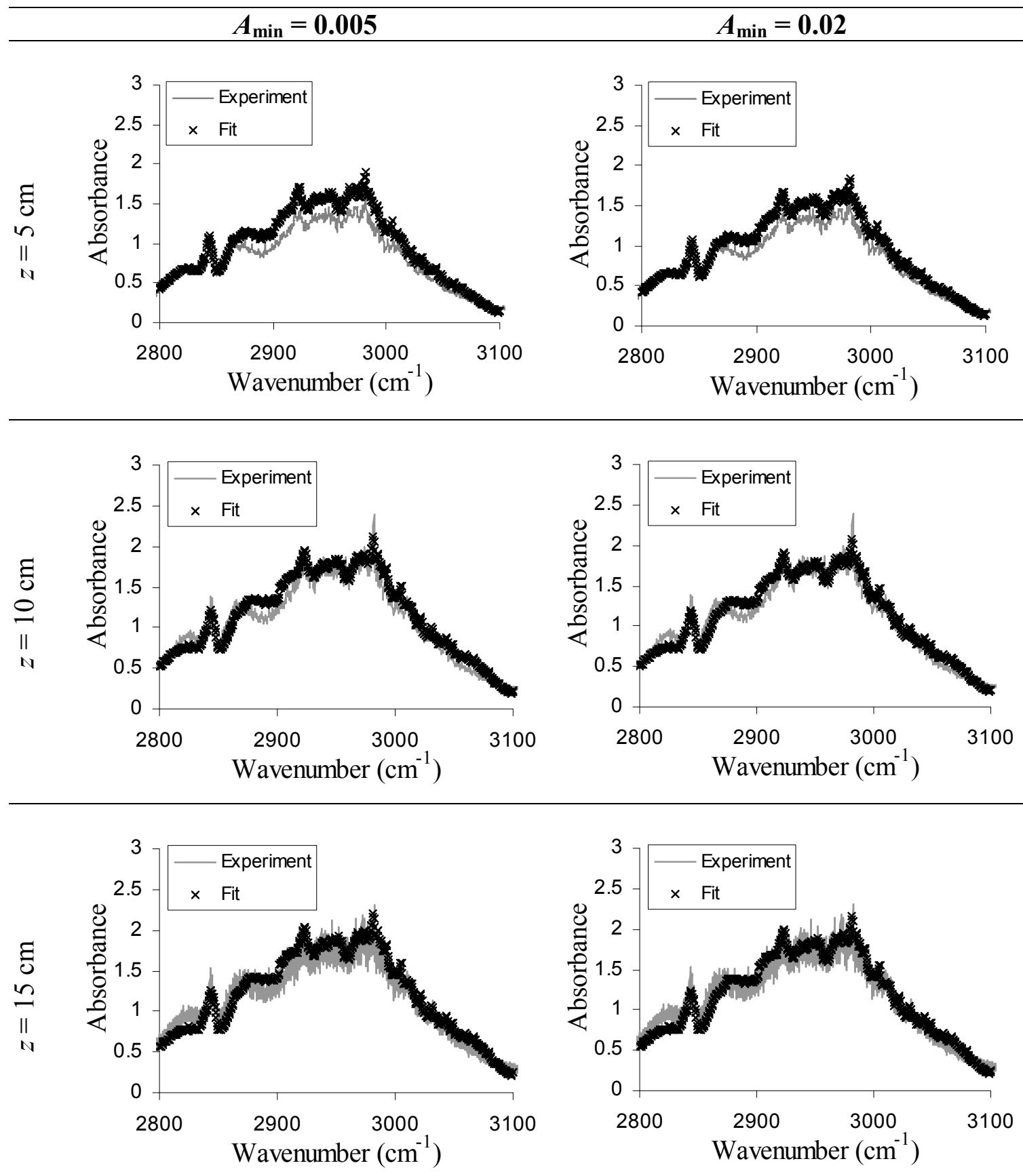

Figure 4.14: $\mathrm{CH}_{3} \mathrm{OH}$ spectra fits to experiment with $80 \%$ decay condition, $T=T_{\text {mod }}$, for $A_{\text {min }}=0.005,0.02$

The results for the spectral fits for $\mathrm{CH}_{3} \mathrm{OH}$ show that the quality of the fit depends greatly upon knowing the temperature profile in the high temperature gradient, fuel-rich core of the pool fire. Unfortunately, there is a lack of high-resolution experimental data 
in this region, and the effect of the experimental test rig used in this experiment was not quantified by thermocouple data. However, several presumptions were made regarding the temperature profiles along the experimental pathlength. When applied to the optimization solver, the shapes of the fitted spectral bands qualitatively improved, as compared to the experimental absorbance spectra. While this is encouraging for the viability of using infrared spectroscopy to study the composition of pool fires, more refined local temperatures data is needed to provide an adequate study of the absolute mole fractions of species near the pool surface.

The $\mathrm{CH}_{3} \mathrm{OH}$ mole fractions predicted above $\mathrm{z}=5 \mathrm{~cm}$ suffered from a lack of experimental absorbance data points. The numerical solver was not naturally able to solve for concentrations in $1 \mathrm{~cm}$ increments based upon experimental spectra measured at $5 \mathrm{~cm}$ increments; the solver had no basis for fitting these points and only cared about the average effect over the $5 \mathrm{~cm}$ measurement span. The problem is not as pronounced for the mole fraction fit for the first $z=5 \mathrm{~cm}$ above the flame because the rapidly changing temperature profile has a large effect on the band shapes. The only manner in which the solver predicted properly shaped mole fraction profiles was to constrain the solution. This was applied with some success in the fits for $\mathrm{CH}_{3} \mathrm{OH}$, but more experimental absorbance measurements are needed at various heights if mole fraction fits are to be calculated for species where the concentration profiles are not as predictable. 


\subsubsection{Carbon Dioxide $\left(\mathrm{CO}_{2}\right)$ and Carbon Monoxide (CO)}

The C-O bands chosen for fitting concentrations of $\mathrm{CO}$ and $\mathrm{CO}_{2}$ (Table 4.1)

overlap substantially, especially when high-temperature broadening of $\mathrm{CO}_{2}$ is considered.

Plots of the high-temperature absorption coefficients for $\mathrm{CO}$ and $\mathrm{CO}_{2}$ are presented in

Figure 4.15 and Figure 4.16, respectively. These figures show that overlapping is a concern for high-temperature spectra fits. In this study, two approaches to handle the overlap will be investigated. First, the spectra fits will be solved simultaneously for both spectra in the $2000-2400 \mathrm{~cm}^{-1}$ range, to estimate the combined absorbances of the two bands. A second method use narrower spectral regions to fit the concentrations individually for the two species.

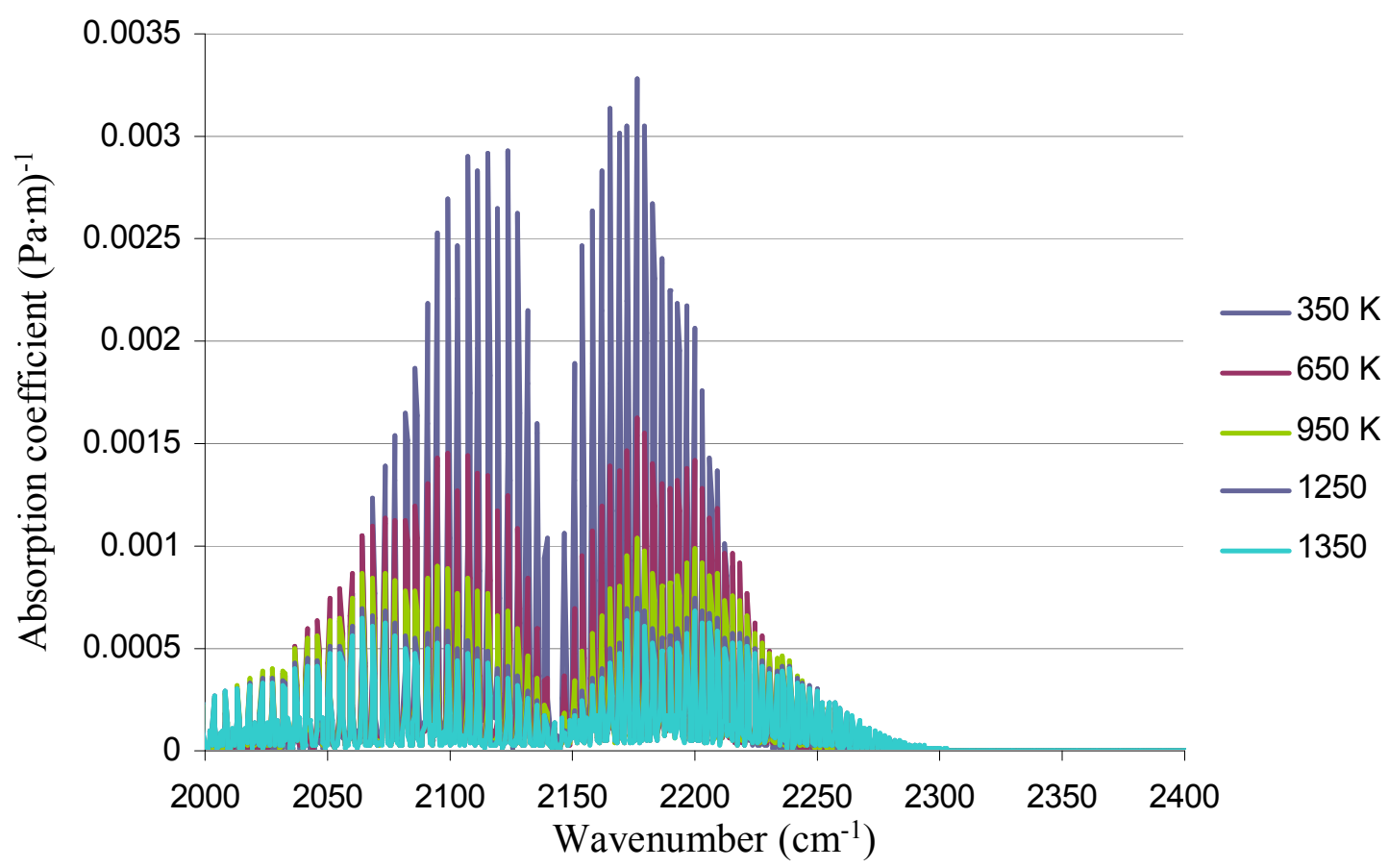

Figure 4.15: Temperature-dependent absorption coefficients of $\mathrm{CO}$ 


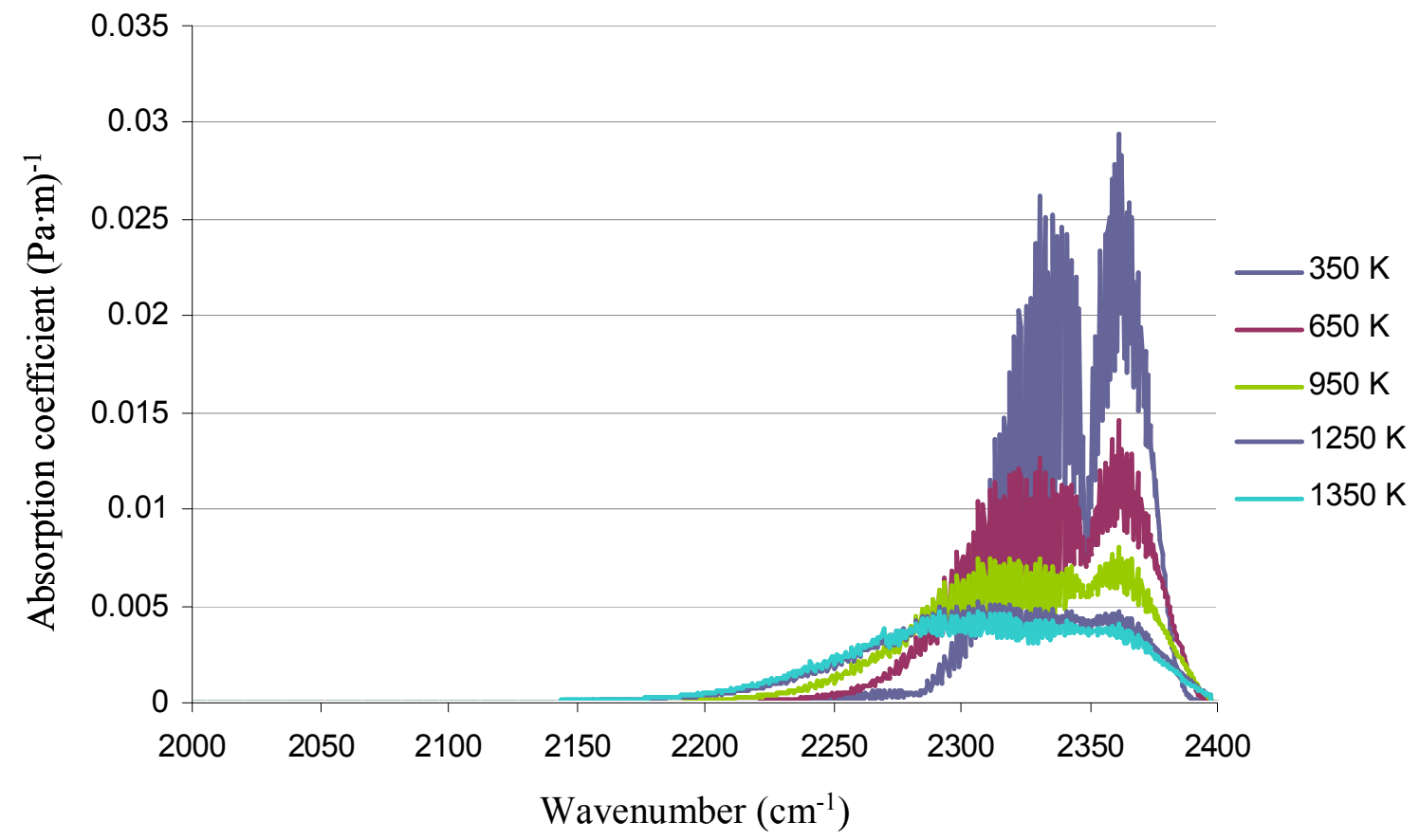

Figure 4.16: Temperature-dependent absorption coefficient for asymmetric stretching band of $\mathrm{CO}_{2}$

The figures show the overlap of the two bands at high temperatures between 2200 and $2280 \mathrm{~cm}^{-1}$. The $\mathrm{C}-\mathrm{O}$ bands used by the solver to fit for $\mathrm{CO}$ and $\mathrm{CO}_{2}$ concentrations individually excluded this region to avoid having to fit where the absorbances are cumulative. For independent $\mathrm{CO}$ and $\mathrm{CO}_{2}$ studies, the wavenumber regions of interest are given in Table 4.2 . 
Table 4.2: Wavenumber regions used for $\mathrm{CO}$ and $\mathrm{CO}_{2}$ when solved independently

\begin{tabular}{|c|c|}
\hline Compound & Wavenumber Region \\
\hline $\mathrm{CO}_{2}$ & 2280 to $2400 \mathrm{~cm}^{-1}$ \\
\hline $\mathbf{C O}$ & 2000 to $2200 \mathrm{~cm}^{-1}$ \\
\hline
\end{tabular}

It is computationally helpful to set realistic boundaries on the species profiles $X_{\mathrm{CO}}$ and $X_{\mathrm{CO} 2}$ in order to guide and accelerate the fit calculations. The boundary constraints on the $X_{\mathrm{CO}}$ and $X_{\mathrm{CO} 2}$ values can be guided by the relevant $\mathrm{GC}$ measurements. The maximum mole fractions recorded for $\mathrm{CO}_{2}$ and $\mathrm{CO}$ from the $\mathrm{GC}$ were 0.054 and 0.064 , respectively. To be conservative and to allow for play in the code, an upper bound of $X=$ 0.20 was chosen for both species. Another constraint that proved useful was limiting the spatial concentration gradient in the solution. This reduced the "noise" created in the solution when the MATLAB function selected random local species concentrations to provide the best fit to the experimental spectra. This was accomplished by "guiding" the code, i.e. limiting the relative (percent) change in each $1 \mathrm{~cm}$ step increment. The results using two different constraints will be compared: 


$$
\begin{array}{ll}
\text { Constraint 1: } & \frac{1}{2}<\frac{X_{k, i}}{X_{k, i+1}}<2 \\
\text { Constraint 2: } & \frac{1}{1.5}<\frac{X_{k, i}}{X_{k, i+1}}<1.5
\end{array}
$$

Figure 4.17: Constraints used by the spectral optimization solver for $\mathrm{X}_{\mathrm{CO}}$ and $\mathrm{X}_{\mathrm{CO} 2}$ profile fits

Based on the insight gained about temperature profiles near the fuel surface from the parametric study conducted for $\mathrm{CH}_{3} \mathrm{OH}$ fits, the modified temperature profile, $T_{\text {mod, }}$ presented in Figure 4.10 presumably provides the most accurate profile of temperature in the fuel-rich core, and will be used to calculate most of the absorbance spectra fits to experimental absorbance bands for $\mathrm{CO}$ and $\mathrm{CO}_{2}$. However, it is still beneficial to run an absorbance fit with the original temperature model to compare how the $\mathrm{CO}$ and $\mathrm{CO}_{2}$ absorbance band shapes change with cooler temperatures in the fuel core. This case was run with $A_{\min }=0.01$, using "Constraint 1 " to define the shape of the mole fraction fits, fitting for absorbance spectra measured up to $z=15 \mathrm{~cm}$. The result of the mole fraction fit difference between $T_{\text {orig }}$ and $T_{\bmod }$ is shown in Figure 4.18, and the fitting to the experimental spectra using $T_{\text {orig }}$ and $T_{\bmod }$ are shown in Figure 4.19 and Figure 4.20, respectively. Fitting with $T_{\text {mod }}$ predicts lower concentrations of $\mathrm{CO}_{2}$ near the fuel surface than $T_{\text {orig. }} . T_{\text {mod }}$ also creates a better fit to the band shape around the central peak $\mathrm{CO}_{2}$, in the region between 2300 and $2375 \mathrm{~cm}^{-1}$, particularly for the absorbance spectra measured and calculated at $z=5 \mathrm{~cm}$. These spectra continue to suggest that the $T_{\bmod }$ profile more adequately represents the experimental conditions. 


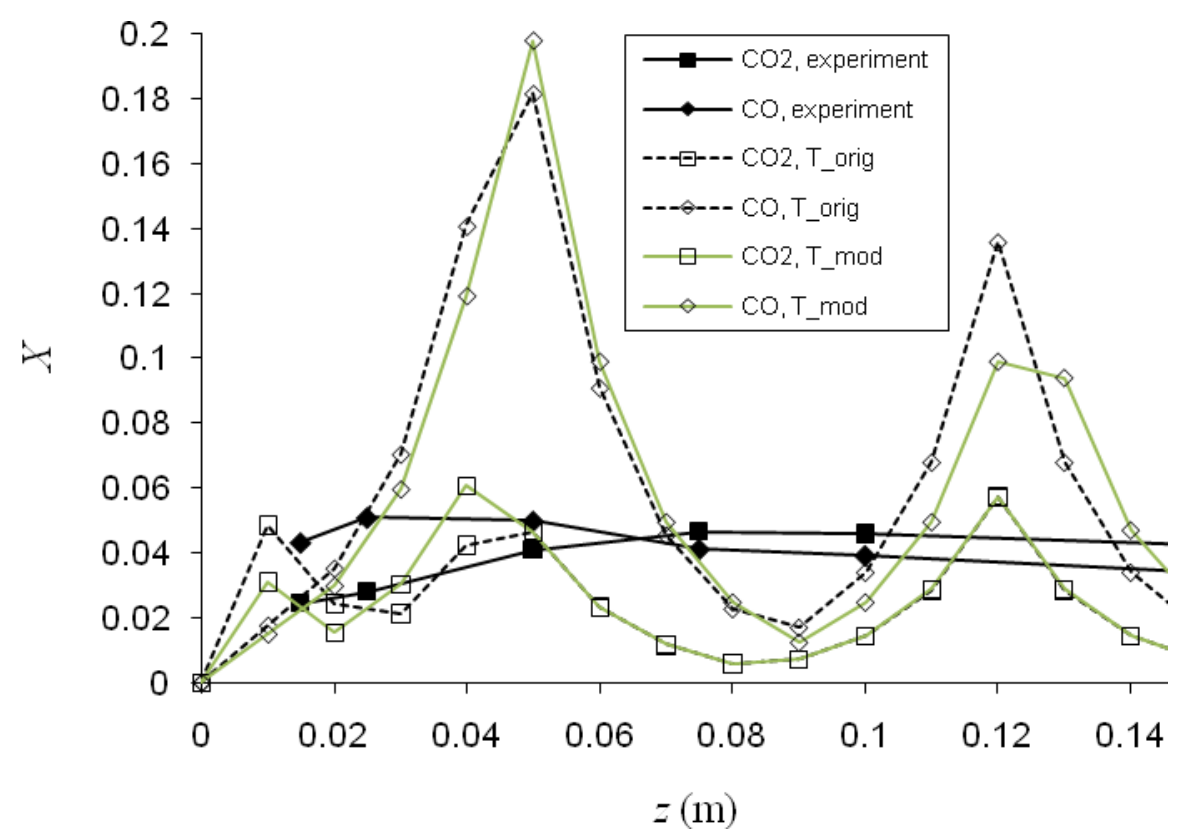

Figure 4.18: Comparison of model fits for $\mathrm{X}_{\mathrm{CO} 2}$ and $\mathrm{X}_{\mathrm{CO}}$ using $T_{\text {orig }}$ and $T_{\mathrm{mod}}$ temperature profiles

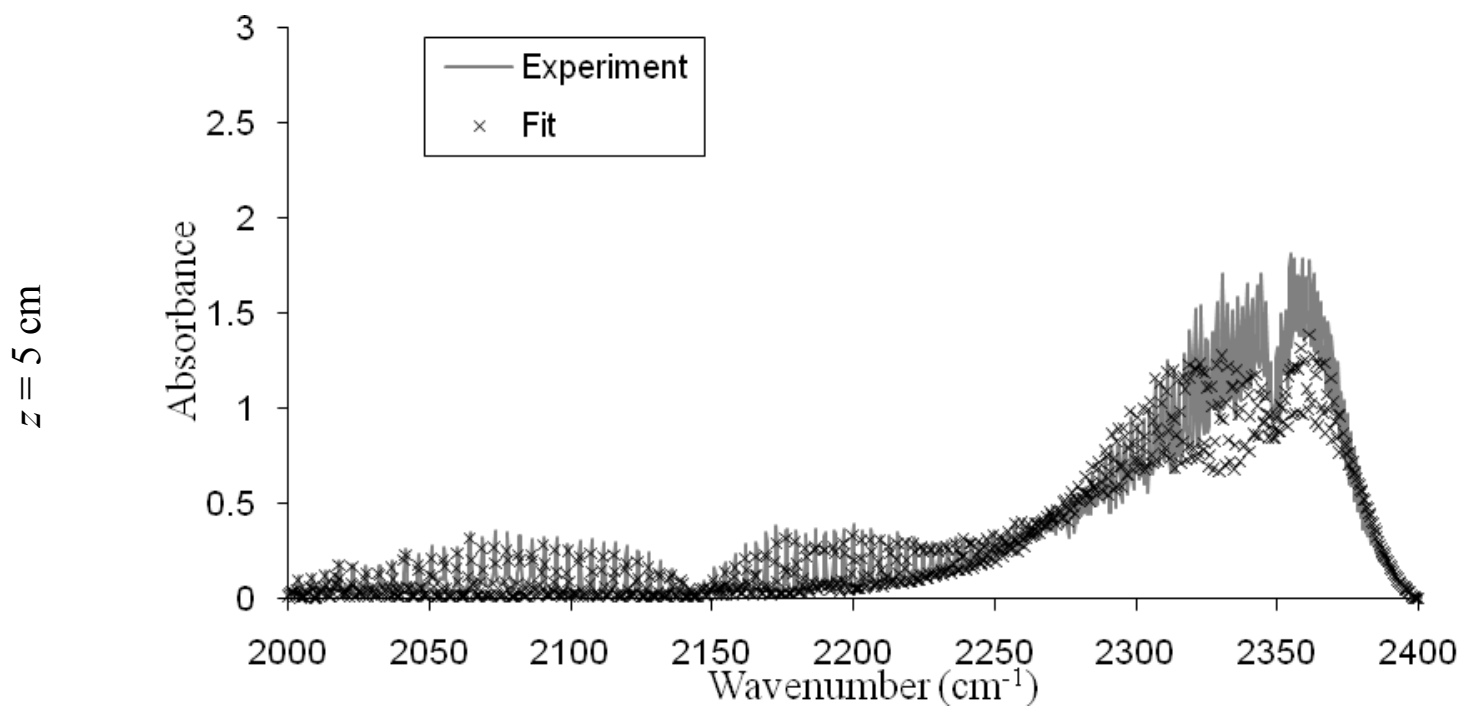



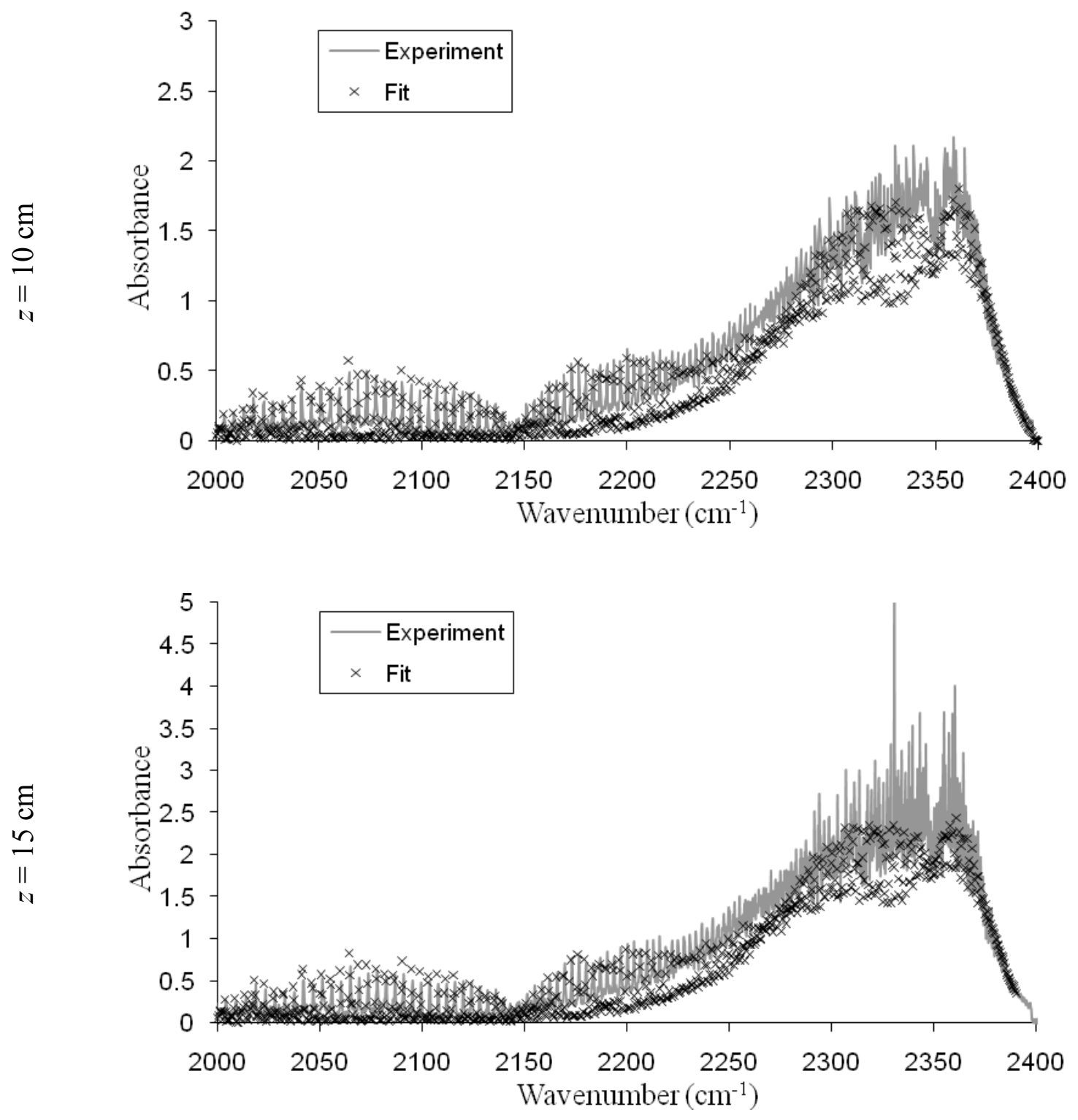

Figure 4.19: $\mathrm{CO}$ and $\mathrm{CO}_{2}$ spectra fits in 2000 to $2400 \mathrm{~cm}^{-1}$ region compared to experiments through $z=15 \mathrm{~cm}$ using $T_{\text {orig }}$ 

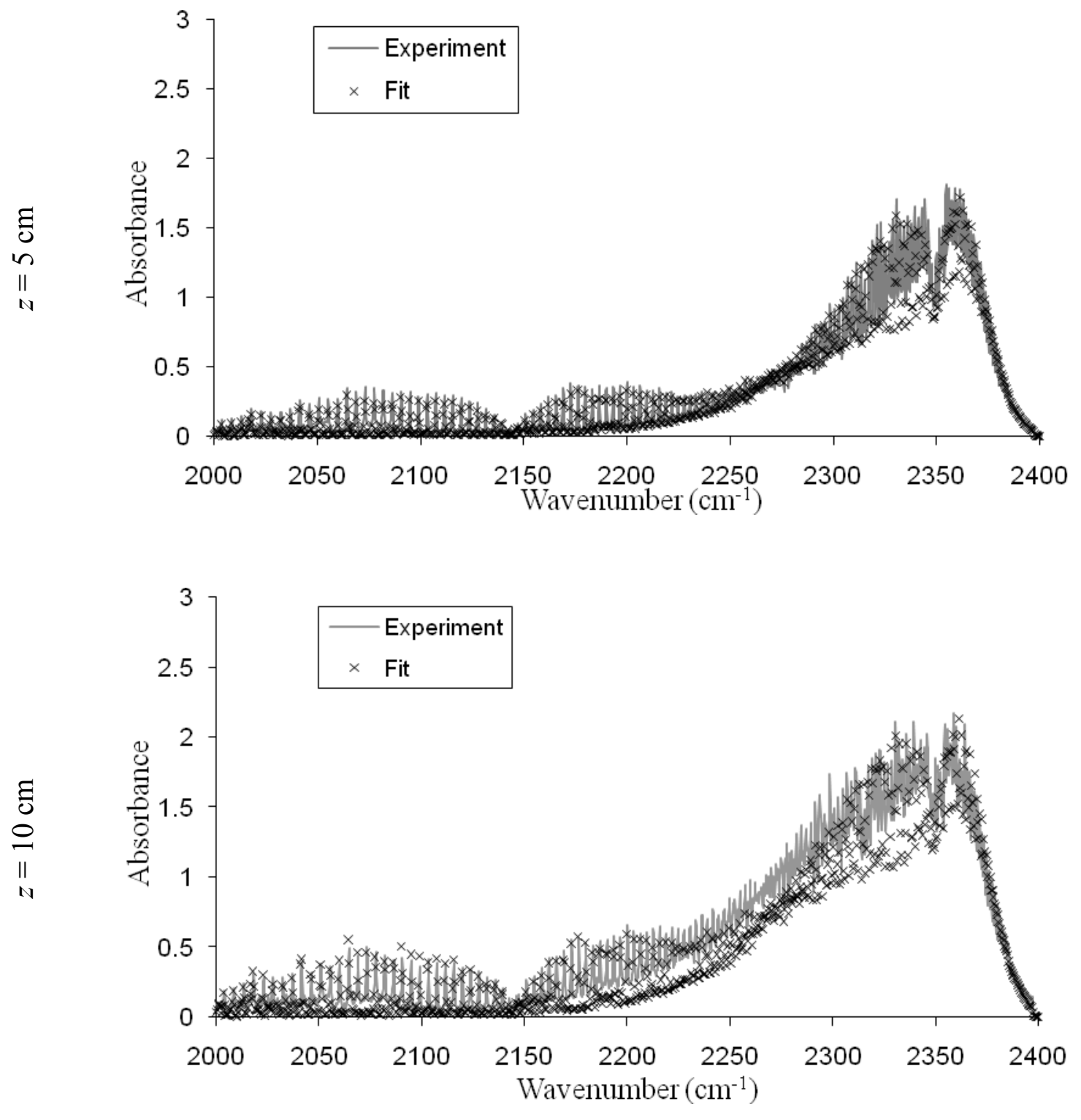


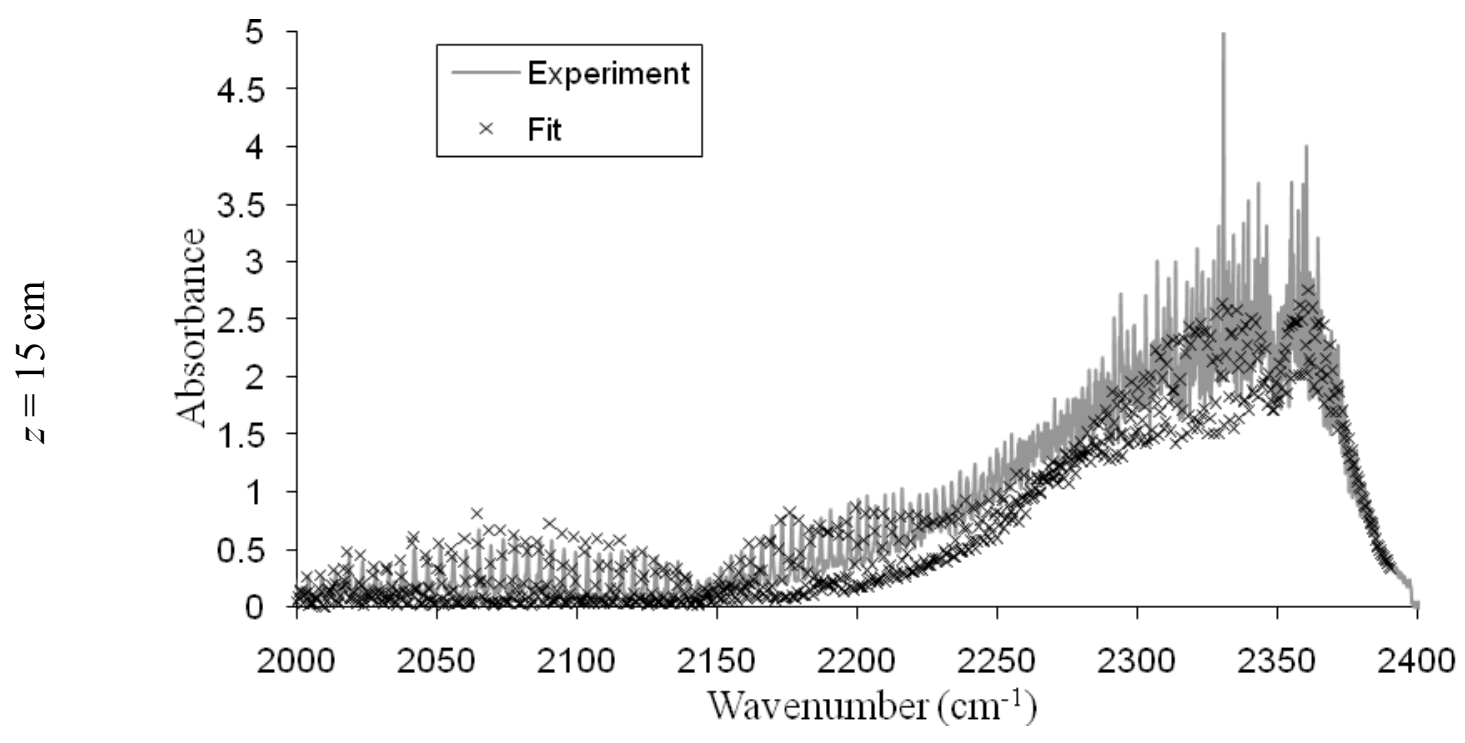

Figure 4.20: $\mathrm{CO}$ and $\mathrm{CO}_{2}$ spectra fits in 2000 to $2400 \mathrm{~cm}^{-1}$ region compared to experiments through $z=15 \mathrm{~cm}$ using $T_{\bmod }$

One problem with the combined fit is that an underestimation of high-temperature broadened $\mathrm{CO}_{2}$ absorbance can create an underestimate of the "baseline" for $\mathrm{CO}$ absorption in the overlapping region between 2200 and $2280 \mathrm{~cm}^{-1}$. The preceding figures show that broadened $\mathrm{CO}_{2}$ absorbance was less than what was experimentally recorded. In this case, the spectral optimization solver may overestimate the values of the $\mathrm{X}_{\mathrm{CO}}$ fit to make up for the low $\mathrm{CO}_{2}$ absorbance. To evaluate how the solver handles this scenario, the $\mathrm{X}_{\mathrm{CO}}$ and $\mathrm{X}_{\mathrm{CO} 2}$ fits were solved independently in the wavenumber regions given in Table 4.2, and are plotted in Figure 4.21. "Constraint 2" was used. The predicted absorbance fits for the regions used are shown in Figure 4.22. The plots of $\mathrm{X}_{\mathrm{CO}}$ and $\mathrm{X}_{\mathrm{CO} 2}$ in this case are once again not qualitatively different than when solved independently, though the profile shapes are tighter due to the more stringent constraint. The solver produces spectral fits of similar quality to those in the case where spectra were solved 
simultaneously, illustrating that the narrower bands contained sufficient information to solve for both species.

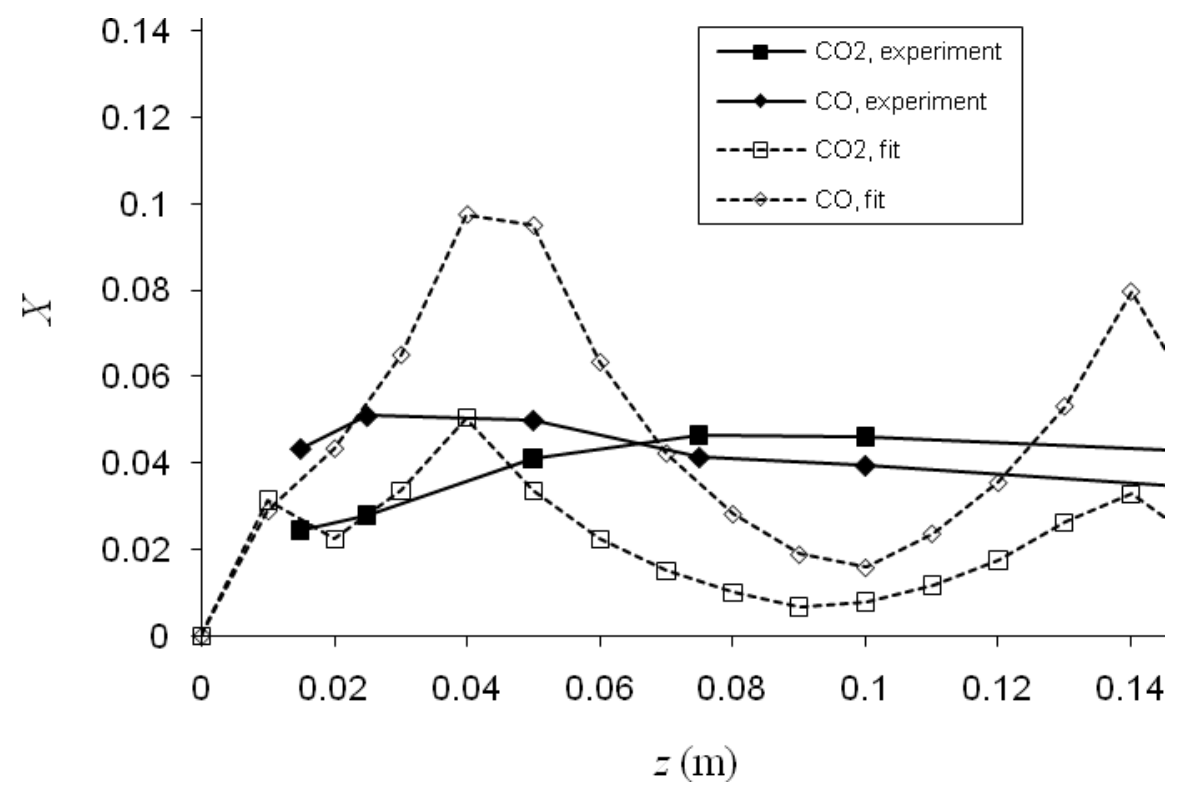

Figure 4.21: Model fits for $\mathrm{X}_{\mathrm{CO} 2}$ and $\mathrm{X}_{\mathrm{CO}}$ when solved in independent spectral regions

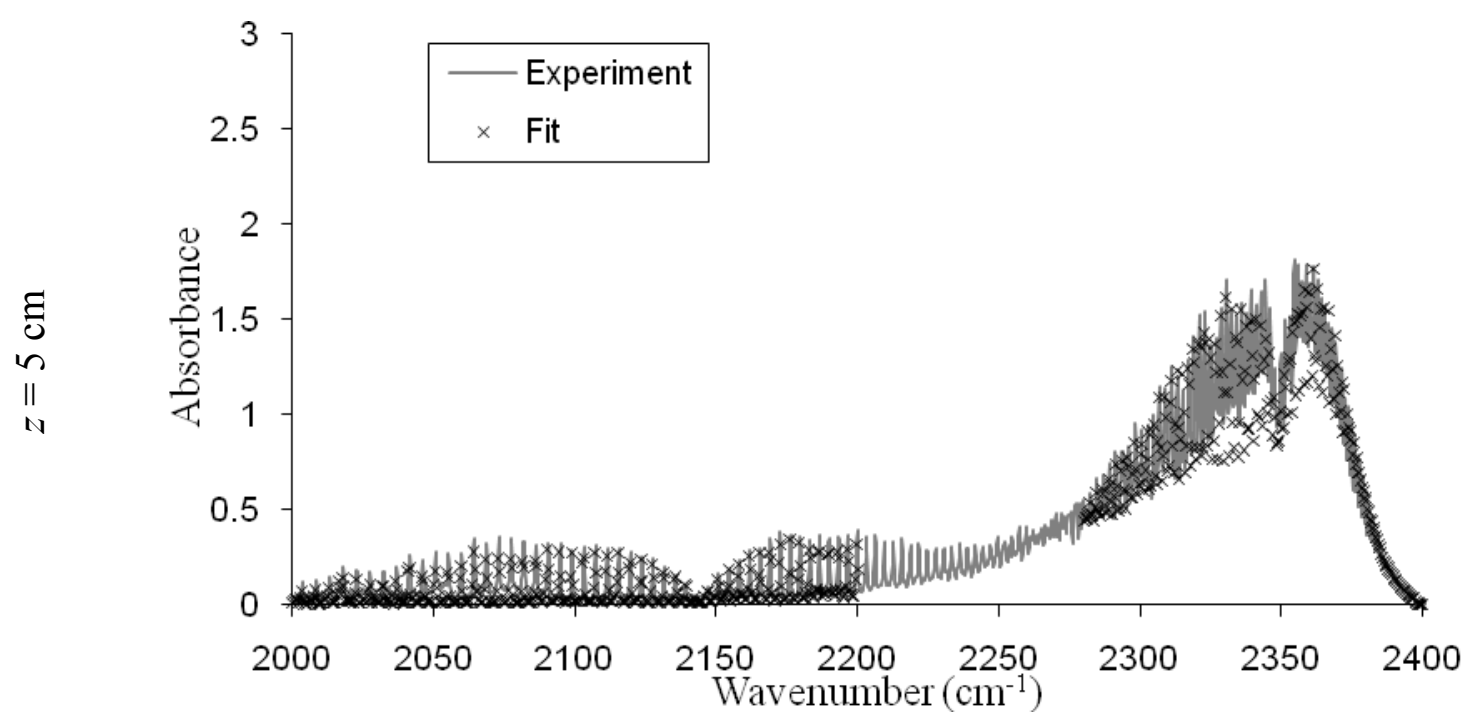



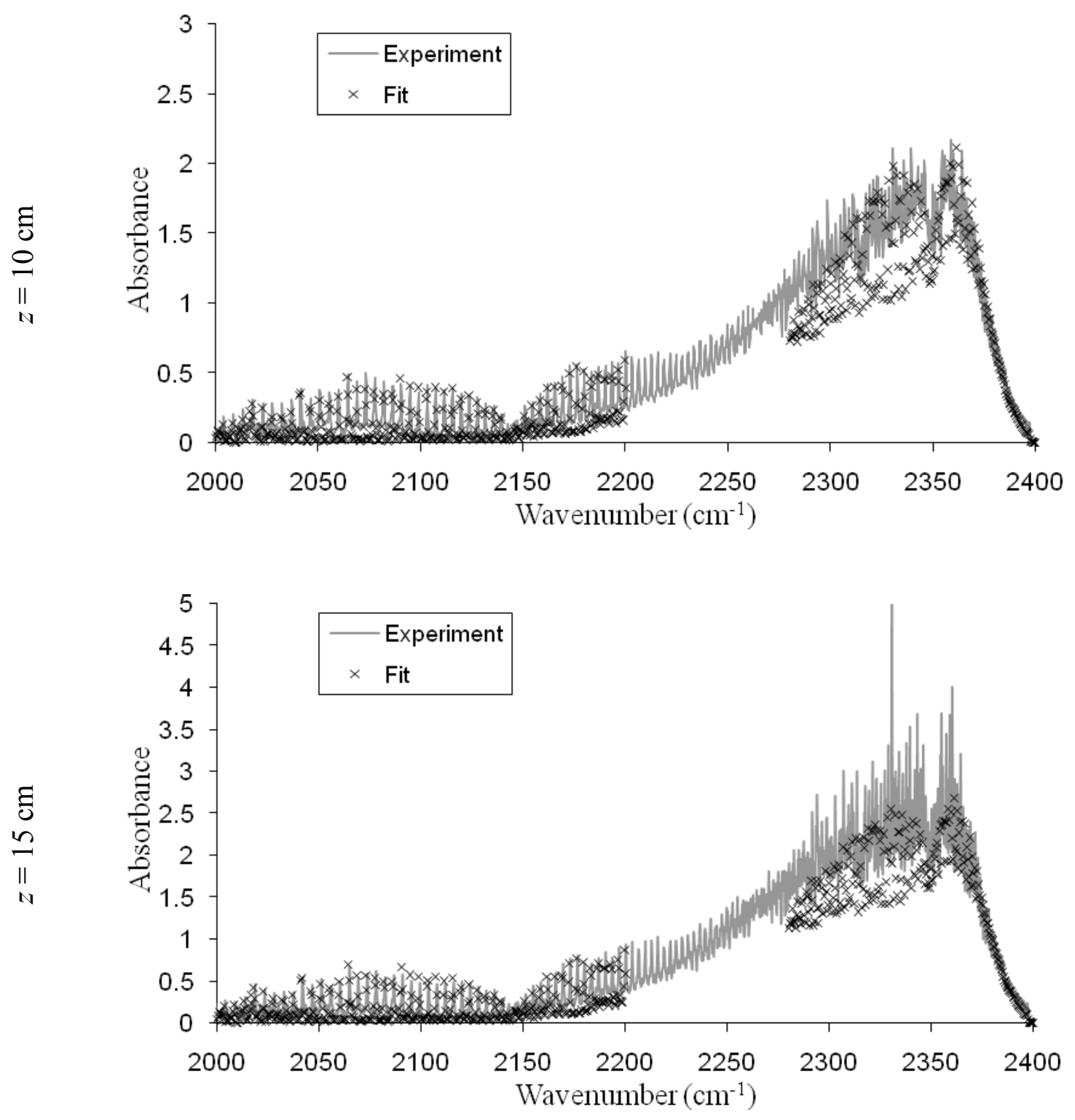

Figure 4.22: Modeled absorbance spectra fits for $\mathrm{CO}_{2}$ and $\mathrm{CO}$ bands when solved independently

One of the problems in the optimization algorithm is related to the lack of spatial resolution of absorbance spectra measurements, as discussed in the section describing $\mathrm{CH}_{3} \mathrm{OH}$ fits. This causes the $\mathrm{X}_{\mathrm{k}}$ fits to vary randomly between the $z_{\text {meas }}$ points. As was done for $\mathrm{X}_{\mathrm{CH} 3 \mathrm{OH}}$ fitting, changing the constraint condition is one method of controlling 
the noise in the fits associated with lack of spatial resolution. "Constraint 2" forces a more controlled profile shape, though this condition is not as stringent as the decay profiles that were able to be applied for $\mathrm{CH}_{3} \mathrm{OH}$; $\mathrm{CO}$ and $\mathrm{CO}_{2}$ are sourced by reactions inside the flame, as such, a simple decay profile could not be assumed. Therefore, the $X$ fits for $\mathrm{CO}$ and $\mathrm{CO}_{2}$ were more difficult to control and became noisier than the experimental results.

As in the previous section, the effects of reducing $A_{\min }$ from the default of 0.01 to 0.005 will be explored in the case using $T_{\text {mod }}$ for $\mathrm{CO}$ concentration fits only; for $\mathrm{CO}_{2}$, $A \gg A_{\min }$ through much of the absorption band. The $\mathrm{CO}$ band has shallow troughs between its peaks where $A \sim A_{\min }$. The spectra are fitted up to $z=15 \mathrm{~cm}$. The fits for the two cases of $X_{\mathrm{CO}}$ are shown in Figure 4.23 and the comparison of absorbance spectra fits are shown in Figure 4.24. As in the case for the $\mathrm{CH}_{3} \mathrm{OH}$ fits, there is very little influence of changing the $A_{\min }$ parameter for the $\mathrm{CO}$ fits. There is a slight change in the spectra calculated for $z_{\text {meas }}=5 \mathrm{~cm}$, though the value of $A_{\text {min }}=0.01$ provides a better fit than the smaller value of 0.005 . Also, the profile of $\mathrm{X}_{\mathrm{CO}}$ is slightly less noisy than for the larger value of $A_{\min }$. This result suggests that this parameter does serve to eliminate some noisy data points that disrupt the fits. 


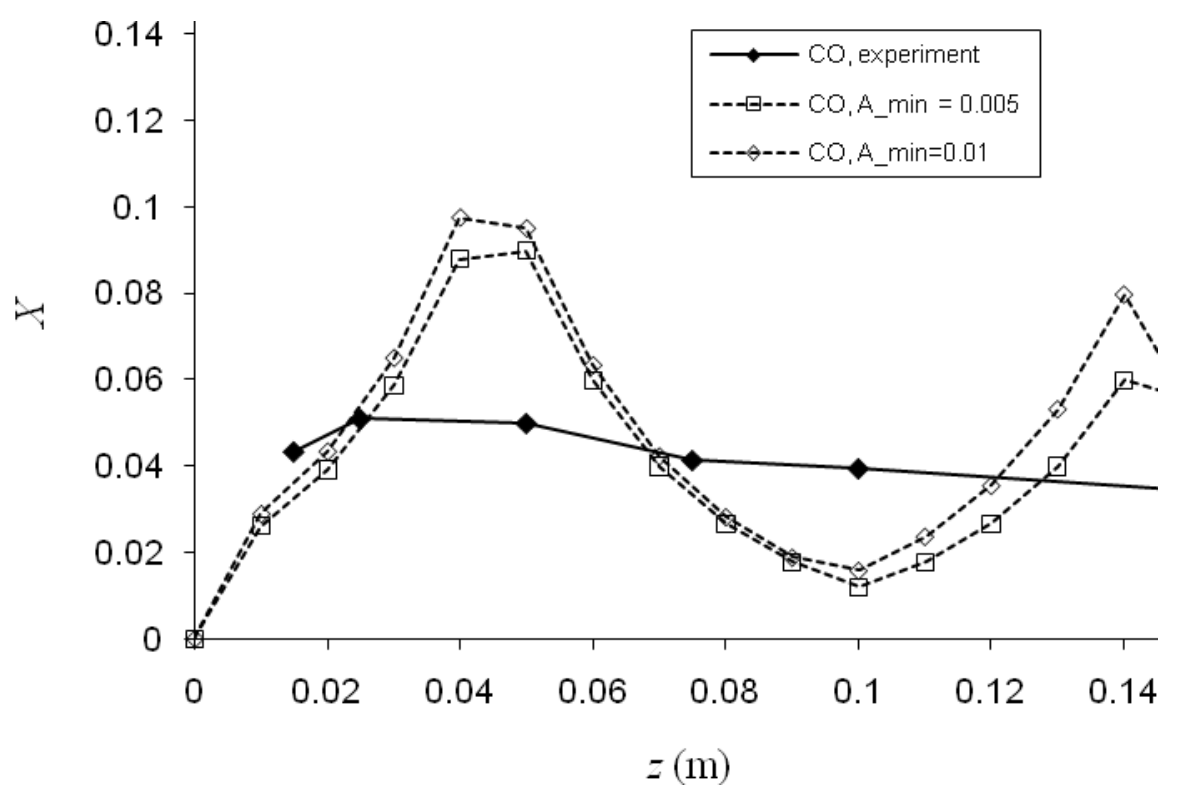

Figure 4.23: A comparison of model fits for $\mathrm{X}_{\mathrm{CO}}$ using two different values of $A_{\min }$ 


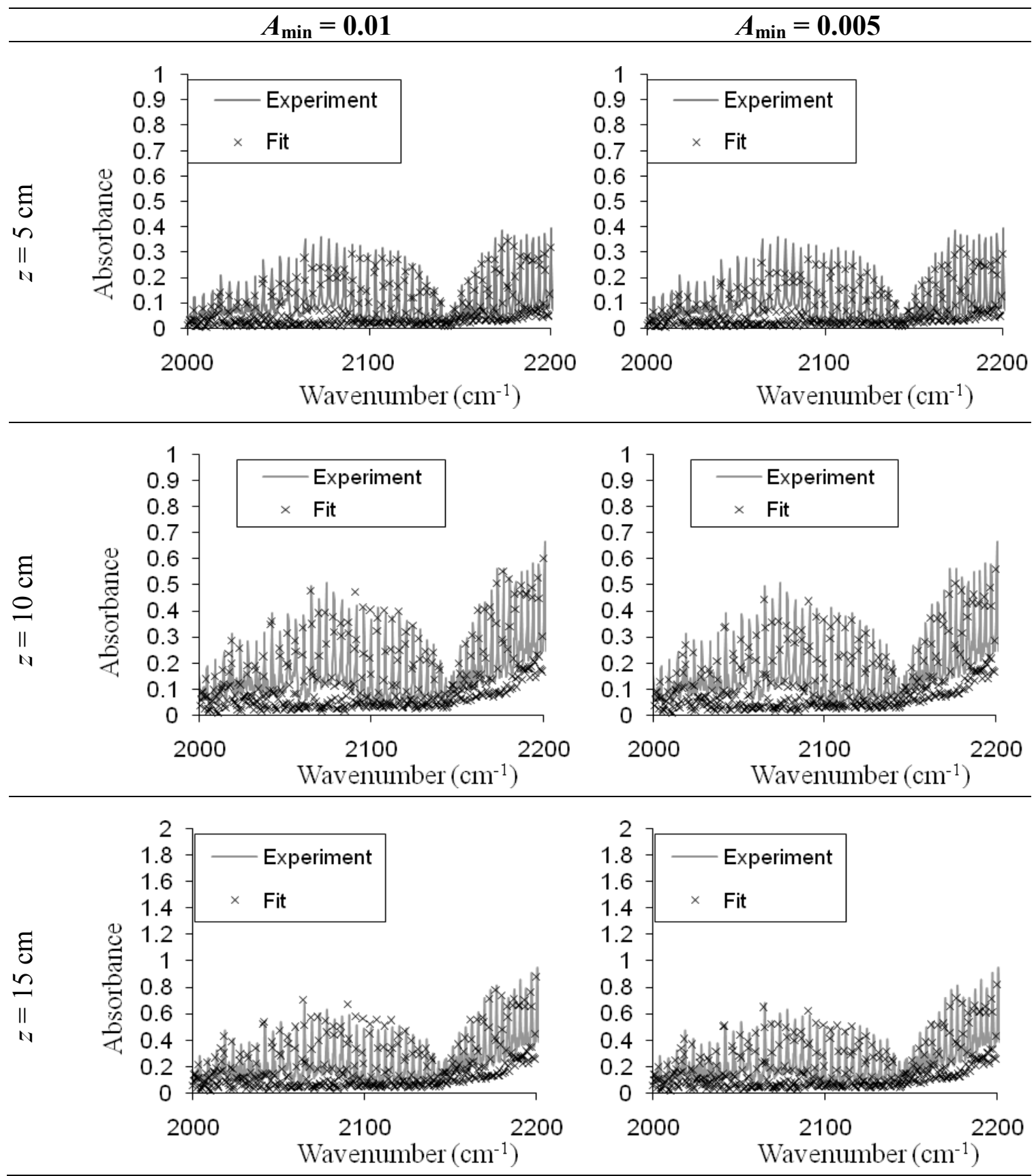

Figure 4.24: Modeled absorbance spectra fit for $\mathrm{CO}$ band using $A_{\min }=0.01,0.005$ 


\subsection{Conclusions}

The spectral solver shows potential as a tool for measuring concentration profiles inside pool fires. The results were shown to be highly sensitive to high temperature gradients found inside the fuel-rich core of the flame, which had significant impacts on the absorbance spectra fits for $\mathrm{CH}_{3} \mathrm{OH}$. Because of the placement error and uncertainty associated with the temperature measurements, some assumptions were made about the temperature profile near the surface of the pool. Spectra modeled using a modified temperature profile provided circumstantial evidence that the presence of the stainless steel cone and its $\mathrm{N}_{2}$ purge displaced the concentration and temperature profiles along the experimental pathlength of the flame. This phenomenon requires better understanding if this experimental method is to be used to produce reliable data on absolute species concentrations inside a pool fire.

A proper analysis of the $d=30 \mathrm{~cm}$ methanol pool fire in question would have benefited from additional experimental data points. The calculator had problems fitting concentrations at $1 \mathrm{~cm}$ increments while the experiment was measured at $5 \mathrm{~cm}$ increments. The only exception was the fit for $\mathrm{CH}_{3} \mathrm{OH}$ profiles in the low $T$ region near the pool surface, in which the high temperature gradient had a very large impact in the band shape. Where applicable, the mole fraction fits benefited from an application of logical constraints on the allowed profile shape of the fit; this provided the most benefit to the $\mathrm{CH}_{3} \mathrm{OH}$ results. It was shown that the $\mathrm{CH}_{3} \mathrm{OH}$ absorption coefficients available for the study were too noisy at high temperatures to be used in the C-O region; an alternate analysis using the $\mathrm{C}-\mathrm{H}$ stretching band was required. In general, this study shows 
promise for the method, though higher data resolution and better temperature data would be of great benefit to acquiring accurate, quantitative results from this type of study. 


\section{CHAPTER 5: Conclusion}

A study was untaken to develop an experimental method to measure the composition of a $d=30 \mathrm{~cm}$ pool fire to better understand the interactions between combustion species and radiative heat transfer within a turbulent diffusion flame. The study of radiative heat transfer is crucial to computational fire models because of its importance determining the size and structure of large fires via determination of vaporization rates.

A unique experimental test rig was designed and constructed to measure in situ infrared absorbance spectra through the centerline of $d=30 \mathrm{~cm}$ pool fires using an FT-IR spectrometer. The test rig consisted of a fuel pan with a centric hole and $\mathrm{N}_{2}$ purge probes to control the experimental pathlength of a frequency-modulated blackbody source. In this effort, a number of unique designs to manage the boundaries of the optical pathlength were implemented. Temperatures profiles within the flame and fuel burn rates were recorded concurrently with spectra measurements. This data provided verification of experimental controls and, in the case of temperature, provided an experimental basis for a parameter required by a computational solver of the radiative transfer equation. Additionally, concentration measurements inside the flame were recorded experimentally with a gas chromatograph (GC) for a comparison with a numerical model to calculate concentrations. A numerical procedure to compensate for transient instrument responses in the FT-IR was developed.

Concentration profiles along the centerline of the $d=30 \mathrm{~cm}$ methanol pool fire were calculated by utilizing Wakatsuki's high-temperature fuel absorption coefficient 
database and the HITRAN database to compare radiative transfer theory against the recorded absorbance spectra. A computer model implementing a discrete form of the radiative transfer equation calculated absorbance spectra along a specified pathlength by using spectral absorption coefficients, local temperature, and local concentrations as inputs. In conjunction with this model, a MATLAB-based optimization code solved for concentration profile fits which best matched up the predicted theoretical spectra to the recorded spectra.

The strong dependence of thermal radiation upon temperature, high temperature gradients, and poorly resolved experimental temperature data created uncertainties when analyzing heat transfer through the fuel-rich core region of the pool fire. This problem was also compounded by low spatial resolution in absorbance data. Some of these problems were overcome by using constraints on the concentration fits and by modeling various absorbance spectra fits using different temperature profiles.

\subsection{Recommendations for Future Research}

This thesis intended to explore the viability of using infrared transmission spectroscopy to measure the composition inside the "fuel-rich core" of pool fires in terms of absolute concentrations. The absorbance spectra measurements, spaced $5 \mathrm{~cm}$ apart, did not provide enough resolution to fully model the fuel-rich region of the flame with the desired resolution and certainty. Research to provide highly resolved temperature measurements in the quickly reacting region near the fuel surface would be useful in predicting radiative transfer through the flame. Better understanding of the temperature 
would have implications on both gaseous species composition studies and heat transfer analyses.

Also, a more comprehensive study of the concentration profile of a pool fire would require more spatial resolution of the measurement spectra. This task would be greatly eased with an improved experimental apparatus. Highly repeatable absorbance spectra measurements at high spatial resolution could be achieved by automating the mechanism controlling the experimental pathlength. This would allow the experimenter to record many measurements at finely controlled increments without interrupting the test, greatly reducing the test times. Reduced test time would also have the added benefit of reducing FT-IR drift. Additionally, a test bed using professional optical mounts would preserve higher signal transmission through the system, which would reduce noise in the experiment and would reduce set up time. Different purge configurations and window materials could potentially eliminate the infiltration of $\mathrm{N}_{2}$ into the measurement pathlength which, while not a large problem at the pathlengths measured in this study, would become an issue with measurements at finer spatial resolution. Specifically, if there is a chemically-resistant window material with high transparency in the IR spectrum, it could be used to cap the cone sitting in the pan without fear of splashing, as a purge clears the non-experimental pathlength underneath the pool surface. Additionally, window materials could be specifically chosen for the absorbance bands being measured, and a new experimental arrangement could make these windows easily interchangeable.

A similar study of various sooty, radiation-dominated fuels would be useful to study the radiation assumptions used in those fuels. The method to correct for gray or gray-like (not line-absorbing) absorption, presented in this thesis for the correction of 
time-dependent instrument responses, would be adequate to subtract the infrared absorption contributed by soot. However, a better characterization of FT-IR spectrometer response or an extremely well calibrated FT-IR spectrometer would be required to quantify the contribution of absorption by soot using in situ spectroscopy.

The algorithm solving concentration profiles could be improved by coupling the solution to a combustion model; the generic solver did not use the physical constraints of combustion embedded in the code. The addition of a combustion model to the code would couple the concentration profiles and the experimental spectra in a way that could lead to accurate and stable fits. 


\section{Nomenclature}

\begin{tabular}{|c|c|}
\hline$A$ & absorbance, $-\ln (\tau)$ \\
\hline$B$ & diffusive transfer number \\
\hline$H$ & enthalpy \\
\hline$I$ & intensity \\
\hline$t$ & moment of inertia \\
\hline$L$ & length \\
\hline$Q$ & heat \\
\hline$P$ & pressure \\
\hline$P_{k}$ & partial pressure \\
\hline$R$ & radius \\
\hline$R F$ & response function \\
\hline$S$ & least-squares fitting function \\
\hline$T$ & temperature \\
\hline$X$ & molar fraction \\
\hline$c$ & speed of light \\
\hline$c_{0}$ & speed of light in a vacuum $=2.9979 \times 10^{8} \mathrm{~m} / \mathrm{s}$ \\
\hline$c_{p}$ & specific heat \\
\hline$d$ & diameter \\
\hline$e$ & photon energy \\
\hline$f_{v}$ & photon distribution function \\
\hline$g$ & acceleration due to gravity, $=9.81 \mathrm{~m} / \mathrm{s}^{2}$ \\
\hline$h$ & Planck's constant or heat transfer coefficient \\
\hline$j$ & angular momentum quantum number \\
\hline$k$ & thermal conductivity \\
\hline$k_{B}$ & Boltzmann's Constant $=1.38 \times 10^{-23} \mathrm{~J} / \mathrm{K}$ \\
\hline$m$ & mass \\
\hline$n$ & unit vector normal to flux surface \\
\hline$n$ & index of refraction \\
\hline$q$ & radiant energy flux \\
\hline $\boldsymbol{r}$ & position vector \\
\hline $\mathbf{s}$ & unit direction vector \\
\hline$v$ & vibrational quantum number \\
\hline$z$ & optical pathlength \\
\hline$\Delta$ & difference \\
\hline$\Omega$ & solid angle \\
\hline$\alpha$ & absorptivity \\
\hline$\varepsilon$ & molecular energy state \\
\hline$\kappa$ & absorbance coefficient \\
\hline$\lambda$ & wavelength \\
\hline$v$ & frequency \\
\hline$\sigma$ & Stefan-Boltzmann constant \\
\hline$\tau$ & transmissivity \\
\hline
\end{tabular}




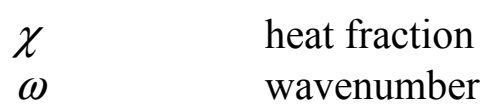

Subscripts:

$\begin{array}{ll}B G & \text { background } \\ b & \text { blackbody } \\ c & \text { combustion } \\ \text { calc } & \text { calculated } \\ \text { cond } & \text { conduction } \\ \text { conv } & \text { convection } \\ f & \text { flame } \\ i & \text { iteration } \\ k & \text { species specific } \\ \text { max } & \text { maximum } \\ \text { meas } & \text { measured } \\ \text { min } & \text { minimum } \\ \text { mod } & \text { modified } \\ \text { norm } & \text { normalized } \\ \text { orig } & \text { original } \\ r & \text { radiation } \\ s & \text { quantity at the pool surface } \\ v & \text { vibration } \\ \text { vap } & \text { vaporization } \\ v & \text { quantity at a particular frequency }\end{array}$




\section{References}

1. V. I. Blinov; G. N. Khudyakov, in: Izdatel'stvo Akademii Nauk SSSR: Moscow, 1961.

2. L. Orloff; J. de Ris, in: Nineteenth Symposium (International) on Combustion, The Combustion Institute: 1982; pp 885-895.

3. R. I. Emori; K. Saito, Combustion Science and Technology 1983, 31, (5-6), $217-$ 231.

4. A. Hamins; S. J. Fischer; T. Kashiwagi; M. E. Klassen; J. P. Gore, Combustion Science and Technology 1994, 97, (1-3), 37-62.

5. A. Hamins; T. Kashiwagi; R. R. Buch in: Characteristics of Pool Fire Burning, Fire Resistance of Industrial Fluids, Indianapolis, IN, 1996; G. E. Totten; J. Reichel, (Eds.) ASTM: Indianapolis, IN, 1996; pp 15-41.

6. D. B. Spalding, Some Fundamentals of Combustion. Batterworth: London, 1955.

7. H. Hottel, Fire Research Abstract Reviews 1959, 1.

8. D. Burgess; M. Hertzberg, in: Heat Transfer in Flames, N. Afgan; J. M. Beer, (Eds.) Scripta Book Company: Washington, 1974; pp 413-30.

9. R. O. Buckius; C. L. Tien, International Journal of Heat and Mass Transfer 1977, 20, (2), 93-106.

10. M. A. Brosmer; C. L. Tien, Combustion Science and Technology 1987, 51, (1-3), 21-37.

11. C. L. Tien, in: Academic Press: New York, 1968; Vol. 5, pp 253-324.

12. L. S. Rothman; D. Jacquemart; A. Barbe; D. C. Benner; M. Birk; L. R. Brown; M. R. Carleer; C. Chackerian; K. Chance; L. H. Coudert; V. Dana; V. M. Devi; J. M. Flaud; R. R. Gamache; A. Goldman; J. M. Hartmann; K. W. Jucks; A. G. Maki; J. Y. Mandin; S. T. Massie; J. Orphal; A. Perrin; C. P. Rinsland; M. A. H. Smith; J. Tennyson; R. N. Tolchenov; R. A. Toth; J. Vander Auwera; P. Varanasi; G. Wagner, Journal of Quantitative Spectroscopy \& Radiative Transfer 2005, 96, (2), 139-204.

13. A. Dayan; C. L. Tien, Combustion Science and Technology 1974, 9, (1-2), 41-47.

14. A. T. Modak, Combustion and Flame 1977, 29, (2), 177-192. 
15. J. de Ris, in: Seventeenth Symposium (International) on Combustion, The Combustion Institute: 1979; pp 1003-1016.

16. A. T. Modak The burning of large pool fires; Factory Mutual: 1979.

17. K. Wakatsuki. High Temperature Radiation Absorption of Fuel Molecules and an Evaluation of its Influence On Pool Fire Modeling. University of Maryland, College Park, MD, 2005.

18. K. Akita; T. Yumoto, in: Tenth Symposium (International) on Combustion, The Combustion Institute: 1965; p 943.

19. S. Hostikka; K. B. McGrattan; A. Hamins in: Numerical Modeling of Pool Fires Using LES and Finite Volume Method for Radiation, Fire Safety Science -Seventh (7th) International Symposium, Worcester, MA, 2003; D. D. Evans, (Ed.) Intl. Assoc. for Fire Safety Science: Worcester, MA, 2003; pp 383-394.

20. K. Wakatsuki; G. S. Jackson; A. Hamins; M. R. Nyden, Proceedings of the Combustion Institute 2007, 31, 2573-2580.

21. E. J. Weckman; A. B. Strong, Combustion and Flame 1996, 105, (3), 245-266.

22. A. Hamins; N. Gorchkov NIST $30 \mathrm{~cm}$ methanol pool fire. http://www2.bfrl.nist.gov/userpages/wmell/plumes.html (October 1),

23. M. Q. Brewster, Thermal Radiative Transfer and Properties. John Wiley \& Sons, Inc.: New York, 1992.

24. S. Clausen, Measurement Science \& Technology 1996, 7, (6), 888-896.

25. C. G. Yin; L. Rosendahl; S. K. Kaer; S. Clausen; S. L. Hvid; T. Hille, Energy \& Fuels 2008, 22, (2), 1380-1390.

26. J. Bak; S. Clausen, Measurement Science \& Technology 2002, 13, (2), 150-156.

27. D. M. Martin; P. J. Medvecz; K. M. Nichols, Applied Spectroscopy 1993, 47, (11), 1898-1906.

28. P. E. Best; P. L. Chien; R. M. Carangelo; P. R. Solomon; M. Danchak; I. Ilovici, Combustion and Flame 1991, 85, (3-4), 309-318.

29. E. Avallone; T. Baumeister, Marks' Standard Handbook for Mechanical Engineers. 9th ed.; McGraw-Hill Book Company: New York, 1987.

30. N. R. Davidson, Statistical mechanics. McGraw-Hill: New York, 1962; p 540 